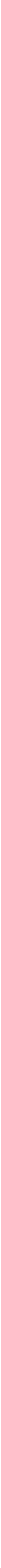

THE GROWTH AND CHARACTERIZATION OF SILICENE, GERMANENE AND HEXAGONAL BORON NITRIDE ADIL ACUN 



\title{
The growth and \\ characterization of silicene, germanene and hexagonal boron nitride
}

\author{
Adil Acun
}




\section{Committee members:}

Chairman and Secretary:

Prof. dr. ir. J.W.M. Hilgenkamp University of Twente

\section{Promotor:}

Prof. dr. ir. H.J.W. Zandvliet

University of Twente

Co-promotor:

Dr. M. Lingenfelder

École Polytechnique Fédérale de Lausanne

Members:

Prof. dr. U. Starke

Prof. dr. C. Kumpf

Prof. dr. ir. G. Koster

Prof. dr. ir. B. Poelsema

Max-Planck Institut für Festkörperforschung Forschungszentrum Jülich University of Twente

Dr. M.P. de Jong

Dr. A. van Houselt

University of Twente

University of Twente

University of Twente

The work and content for this thesis have been carried out at the Physics of Interfaces and Nanomaterials Group, MESA+ Institute for Nanotechnology, University of Twente, Enschede, The Netherlands.

\section{Adil Acun}

The growth and characterization of silicene, germanene and hexagonal boron nitride

ISBN: 978-90-365-4391-0

DOI: $10.3990 / 1.9789036543910$

Cover designed by: Ebru Acun

No part of this publication may be stored in a retrieval system, transmitted, or reproduced in any way, including but not limited to photocopy, photograph, magnetic or other record, without prior agreement and written permission of the publisher. 


\title{
THE GROWTH AND \\ CHARACTERIZATION OF SILICENE, GERMANENE \\ AND HEXAGONAL BORON NITRIDE
}

\author{
Dissertation
}

to obtain

the degree of Doctor at the University of Twente, on the authority of the rector magnificus, Prof. Dr. T.T.M. Palstra,

on account of the decision of the graduation committee, to be publicly defended

on Thursday 14 September 2017 at 14:45 hrs

Adil Acun

born on 22 August 1989

in Deventer, the Netherlands 
This dissertation has been approved by:

Supervisor: Prof. dr. ir. H.J.W. Zandvliet (UT)

Co-supervisor: Dr. M. Lingenfelder (MPI-EPFL) 


\section{Contents}

$\begin{array}{llr}1 & \text { Introduction } & 7\end{array}$

1.1 Introduction to $2 \mathrm{D}$-materials . . . . . . . . . . . 7

1.2 Materials and methods . . . . . . . . . . . . . . . . 8

1.2.1 Low Energy Electron Microscopy . . . . . . . . . . . . . . . 8

1.2.2 Scanning Tunneling Microscopy . . . . . . . . . . . . . . 15

1.2 .3 Theory of STS . . . . . . . . . . . . . . . . 16

1.2.4 Practical setup and Sample Preparation . . . . . . . . . 17

$\begin{array}{ll}\text { Bibliography } & 19\end{array}$

2 Tight-binding method: applied on graphene 23

2.1 Introduction . . . . . . . . . . . . . . . 23

2.2 Crystal structure . . . . . . . . . . . . . . . . 24

2.2.1 Crystal structure of graphene . . . . . . . . . . . . . 24

2.2.2 Crystal structure of silicene and germanene . . . . . . . . . 28

2.2.3 Crystal structure of hexagonal boron-nitride . . . . . . . . 29

2.3 Tight-binding method: first quantization . . . . . . . . . . . . . . 31

2.4 The long wavelength limit approximation . . . . . . . . . . . . 34

2.5 Density of states of graphene . . . . . . . . . . . . . . . . . . . . . . . . . . . . 37

2.6 Dirac equation . . . . . . . . . . . . . . . . . . . . . . . . . . . . 49

2.7 Tight-Binding Graphene-edges . . . . . . . . . . . . . . . . . . . . . . . . . . . . . . . 43

2.7.1 Armchair Edges . . . . . . . . . . . . . . . . . . . . . . . . . 43

2.7.2 Zigzag Edges . . . . . . . . . . . . . . . . . . . 49

2.8 Tight-binding model for hexagonal boron-nitride . . . . . . . . . 56

2.9 Appendix: Tight-binding method; second quantization . . . . . . 56

2.9 .1 Introduction . . . . . . . . . . . . . 56

2.9.2 Applying the second quantization tight-binding method to graphene .................. 58

$\begin{array}{ll}\text { Bibliography } & 61\end{array}$

3 The instability of Si on $\mathrm{Ag(111)} \quad 63$ 


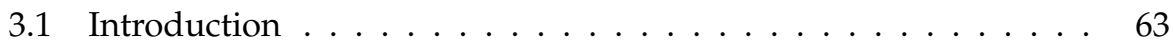

3.2 Materials and Methods . . . . . . . . . . . . . . . . . . . 65

3.3 Si deposition at constant temperature . . . . . . . . . . . 65

3.3.1 Lower growth temperature: different silicene phase . . . . 69

3.4 Annealing silicene: new overlayer structure . . . . . . . . . 71

3.5 X-ray Photoelectron Spectroscopy . . . . . . . . . . . . . . . . . 75

3.6 Conclusion . . . . . . . . . . . . . . . . . 76

$\begin{array}{ll}\text { Appendix A } & \mathbf{7 9}\end{array}$

A.1 Supersaturation potential retrieval $\ldots \ldots \ldots \ldots \ldots$

$\begin{array}{ll}\text { Bibliography } & 83\end{array}$

4 Germanene: a germanium analogue of graphene 87

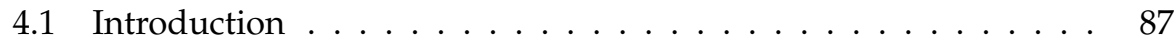

4.2 Theoretical calculations . . . . . . . . . . . . . . . 90

4.3 Synthesis of germanene . . . . . . . . . . . . . . . . . 94

4.3.1 Structural properties on various substrates . . . . . . . . 94

4.3.2 Electronic properties: Scanning Tunneling Spectroscopy

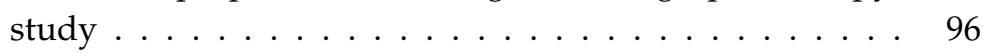

4.4 The future of germanene . . . . . . . . . . . . . . . . 103

4.4.1 Anomalous quantum Hall effect . . . . . . . . . . . 103

4.4 .2 Quantum spin Hall effect . . . . . . . . . . . . . . . 104

4.4 .3 Opening of a band gap in germanene . . . . . . . . 106

4.5 Outlook . . . . . . . . . . . . . . . . . . . . 108

$\begin{array}{ll}\text { Appendix B } & 111\end{array}$

B.1 Derivation of temperature dependent $\mathrm{dI} / \mathrm{dV}$ equation $\ldots \ldots \ldots 111$

$\begin{array}{ll}\text { Bibliography } & 113\end{array}$

5 Real time growth of h-BN on $\operatorname{Ir}(111) \quad 119$

5.1 Introduction . . . . . . . . . . . . . . . . . . . . . . . . . 119

5.2 Materials and Methods . . . . . . . . . . . . . . . . . . 121

5.3 Growth of hexagonal boron nitride . . . . . . . . . . . . 121

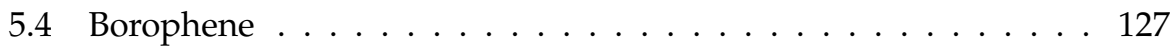

5.5 Conclusion . . . . . . . . . . . . . . . . . . . . 131

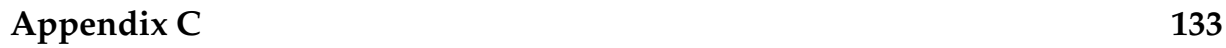

C.1 Step Contrast . . . . . . . . . . . . . . . . . . . 133

C.2 Pressure development . . . . . . . . . . . . . . . 135

C.3 Cold substrate deposition $\ldots \ldots \ldots \ldots \ldots$

C.4 Borophene: LEED . . . . . . . . . . . . . . . . . . . . . . . 137 
Bibliography

Summary

143

Samenvatting

145

List of publications

Acknowledgment

151 



\section{Chapter 1}

\section{Introduction}

\subsection{Introduction to 2D-materials}

Two dimensional materials are crystalline materials possessing a single layer of atoms. In 2004 the first 2D-material was fabricated by mechanical exfoliation of graphite to graphene. For this discovery and the promising exotic properties of graphene the Nobel Prize for Physics was awarded in 2010 [?, ?, ?]. Some notable properties of graphene are Klein tunneling, quantum (spin) Hall effect, anomalous Hall effect and high electron mobilities [?,?,?]. The discovery of graphene initiated a quest to find other 2D-materials similar to graphene: silicene [?], germanene [?] stanene [?], hexagonal boron nitride [?] and transition metal dichalcogenides [?]. Furthermore, other 2D materials that are predicted or found to exist are phosphorene [?,?,?], aluminene [?,?], bismuthene [?] and borophene $[?, ?, ?, ?]$. The list of 2D-materials is still expanding vastly.

In this thesis the emphasis is put on silicene, germanene and hexagonal boron nitride. The thesis starts, after an introduction in the techniques and methods involved in this thesis in Chapter 1.2, with a detailed description of the tightbinding model applied on graphene structures in Chapter 2. The electronic properties of graphene lie in the so-called Dirac cone as retrieved from band structure calculations. Silicene and germanene exhibit Dirac cones similar to that of graphene. The chapter on the tight-binding model is therefore a good appetizer to get familiar with Dirac materials.

During the time span of this PhD-trajectory many novel 2D-materials were discovered. The $s p^{2}$-hybridized 2D-material silicene already made it to existence prior to the PhD-trajectory. The growth of silicene is studied in Chapter3. In the periodic table of the elements silicon is the lower neighbor of carbon. Silicon comprises a similar electronic configuration $\left([\mathrm{Ne}] 3 \mathrm{~s}^{2} 3 \mathrm{p}^{2}\right)$ as carbon $\left([\mathrm{He}] 2 \mathrm{~s}^{2} 2 \mathrm{p}^{2}\right)$. 
It is still an open question whether the silicon analogue of graphene, silicene, exhibits similar structural and electronic properties [?].

Germanene is the topic of Chapter 4. Silicene and germanene do not differ in essence as they both exhibit a buckled honeycomb structure. The buckling height found for germanene is larger $(0.65 \AA)$ [?] than for silicene $(0.44 \AA)$. The buckling results in increments in the spin-orbit coupling [?] and consequently causes a small band gap at the Dirac points. One particular property that arises from the spin-orbit gap is the quantum spin Hall effect. Theoretical calculations have shown that germanene is predicted to have a spin-orbit gap if $23.9 \mathrm{meV}$ [?]. Germanene is therefore a good 2D topological insulator candidate. A literature review on germanene is included in Chapter 4. Furthermore, a scanning tunneling spectroscopy study is provided into the temperature dependency of the electronic properties of germanene.

Silicene and germanene promise extraordinary properties, however, they are typically grown epitaxially on (metal) substrates and thereby altering its properties through the interaction with the substrate. An insulating buffer layer consisting of for instance hexagonal boron nitride (h-BN) might solve this problem. Hence, h-BN on $\operatorname{Ir}(111)$ is studied in the last chapter, Chapter 5, by means of low energy electron microscopy (LEEM). h-BN has a similar structure to graphene differing in its two sub-lattices formed by boron and nitrogen atoms. h-BN is however a wide band gap insulator. This property can be used to use h-BN as a buffer layer to decouple a Dirac material from the substrate. Heterostructural engineering of 2D-materials therefore needs materials as h-BN [?,?,?]. The growth of h-BN on $\operatorname{Ir}(111)$ by means of low energy electron micorscopy is reported in this thesis.

\subsection{Materials and methods}

\subsubsection{Low Energy Electron Microscopy}

Low Energy Electron Microscope (LEEM) is an imaging tool by means of low energetic backscattered electrons developed by Bauer [?,?, ?]. The LEEM is one of the very few nanoscience techniques that is capable of true imaging, i.e. realtime and real-space imaging. This huge advantage is provided by the fact that the electron beam used for imaging illuminates the surface rather than scanning across the surface. The frame rate is limited by the video camera and the sensitivity of the phosphor screen. The latter dependence is a matter of the intensity of the electron beam. Short exposure times, thus large frame rates, are feasible if the intensity of the beam is sufficiently large. The optics required for LEEM also enable LEED (Low Energy Electron Diffraction). The basic optical scheme is given in Fig. 1.1 showing how LEED patterns and LEEM images are created. 
Furthermore, it is also possible to emit electrons via photoemission. This technique is called PEEM (Photoemission Electron Microscopy) and is a powerful way to record large scale images and to study work functions of different materials. Combining LEEM, PEEM and LEED opens a world full of information about growth, nucleation, morphology, work functions, crystal structure and other surface science related phenomena [?,?].

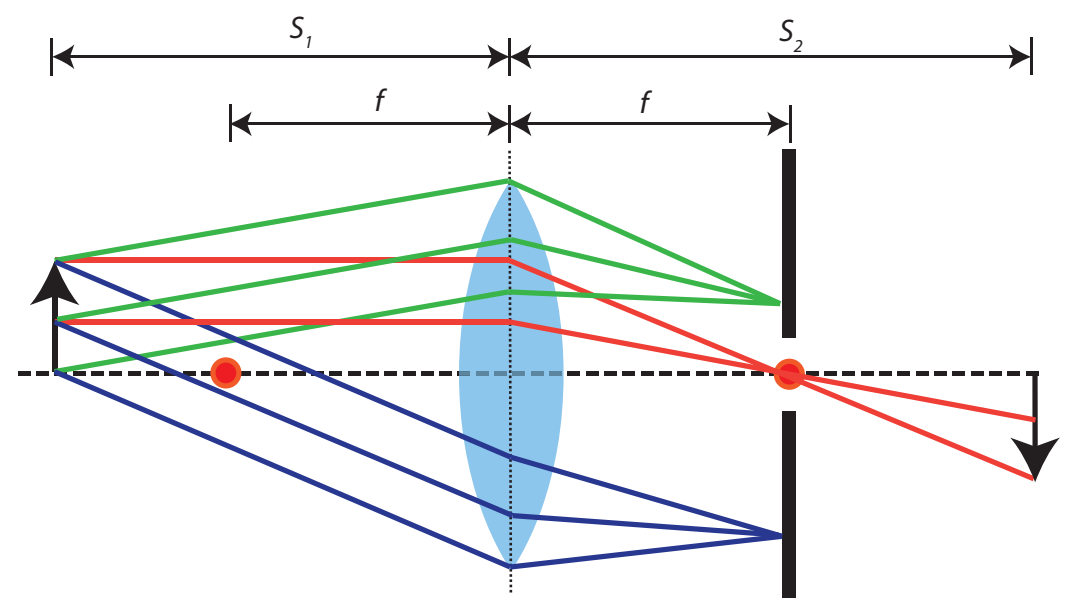

Object

Objective lens Contrast aperture Image

Figure 1.1 - A simplified scheme of the optics in LEEM. Incident electron beams interact with the sample (or object) causing backscattered electrons. The objective lens focuses the backscattered electron beam on the backfocal plane giving rise to a diffraction pattern. The contrast aperture is used to select a single diffraction spot, which is used to form an image of the surface.

\section{Interaction between low energy electrons and matter}

Low energy electrons form the foundation of the LEEM. Electrons are generated at a cathode and an electron beam is emitted towards the sample with a voltage of $20 \mathrm{kV}$. Just before the electrons interact with the sample, the electron energy is reduced such that the electrons have an energy of the order 1 to $100 \mathrm{eV}$. At these low energies the first Born approximation is not valid anymore [?]. Therefore, inelastic scattering and elastic backscattering become more important, while the dominance of forward scattering decreases. Furthermore, at very low energies light atoms backscatter stronger than heavier atoms over a wide energy range. At low energies the dependence of elastic backscattering on nuclear charge is strongly non-monotonic, which is advantageous because this makes it possible to observe light atoms on heavy substrates. Although backscattered electrons in LEEM are in general scattered elastically, this does not hold for all electrons. 
There are several mechanisms for inelastic scattering of these electrons. An incident electron still penetrates a finite distance into the solid bulk and therefore may lose some of its energy. In addition, impurities and defects on the surface may lead to inelastic scattering.

Many inelastic scattering processes involve inner electron shells. However, due to the low energy of the incident electrons it is assumed that incident electrons do not have sufficient energy to interact with the electrons from the inner electron shells of the sample. If there is any inelastic scattering, then it should occur through interaction with the outer shells. Therefore, valence electron excitations determine the attenuation by inelastic scattering. At low energies inelastically backscattering is weaker than elastically backscattering up to a certain threshold energy which is directly related to plasmon excitations. The regime where elastic backscattering dominates over inelastic scattering, typically electron energies up to $20-30 \mathrm{eV}$, is therefore crucial for doing LEEM experiments [?].

\section{Instrumentation}

A schematic of the Elmitec LEEM III setup is shown in Fig. 1.2. A LaB 6 electron gun generates electrons which travel through the illumination column consisting of three condenser lenses. While the first condenser lens demagnifies the crossover, the other two condenser lenses and the beam separator image the cross-over into the back-focal plane of the objective. In the imaging column five lenses are located, namely the transfer lens, field lens, intermediate lens and two projective lenses. The transfer lens images the back-focal plane into the center of the field lens, whereas the field lens in turn images the primary image plane into the object plane of the intermediate lens. The intermediate and projective lenses either image the center of the separator or the back-focal plane. From here the electrons move to the channel plates and are targeted at a fluorescent screen.

The beam separator of the LEEM operates with $60^{\circ}$ deflection [?] as can be seen in Fig. 1.2. Astigmatism and aberrations caused by deflectors are eliminated by realizing equal path lengths in the field and by increasing focusing in the plane normal to the magnetic field. The latter is done by shaping of the magnetic field with inner and outer magnets in the beam separator. Another important component of the LEEM is the objective lens. It produces a virtual image behind the object by a homogeneous electric accelerating field and the magnetic lens produces a real image of the virtual object. The aberration of the electric accelerating field has the largest influence on the resolution [?].

A photograph of the setup is given in Fig. 1.3. The base pressure of the system is ultrahigh vacuum $\left(1 \cdot 10^{-10} \mathrm{mbar}\right)$. The sample can be heated in-situ in the main chamber by heating via a filament (and subsequently electron bombardments) or 


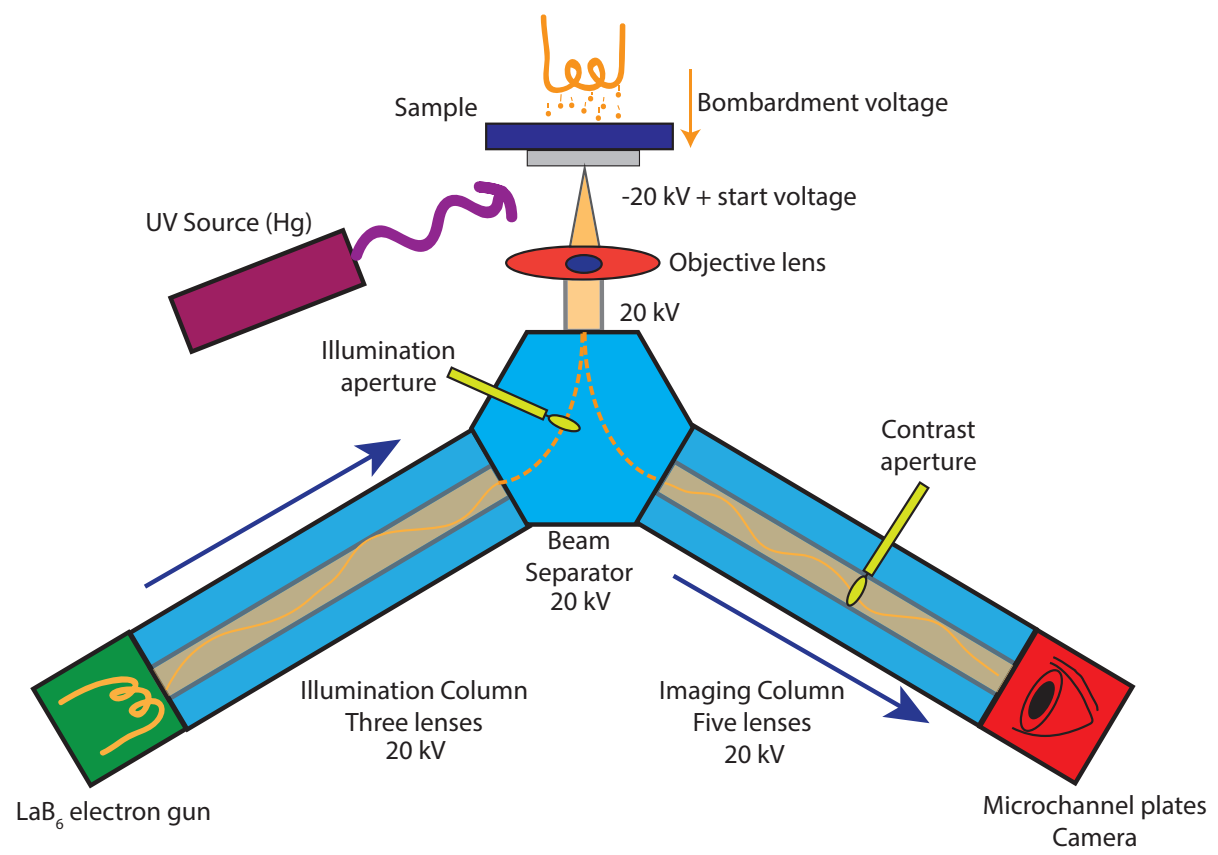

Figure 1.2-Electrons are generated at the electron gun and move with $20 \mathrm{kV}$ through the illumination column and the beam separator. The objective lens focuses the beam on the sample. Between the objective lens and the sample the electrons are decelerated to the start voltage. Backscattered electrons are accelerated again and then return to the beam separator and travel through the imaging column until they are detected by microchannel plates and a CCD-camera. The illumination aperture is able to block the beam down to a diameter of $1.4 \mu \mathrm{m}$ and is used for $\mu$ LEED measurements. The contrast aperture enables to pick a diffraction spot and to image it in real space either in bright-field (specular spot) or dark-field (fractional order spots). Photoemitted electrons can be generated by direct illumination of the sample with UV-light from Hg-discharge source.

can be cooled down by a cryostat and liquid nitrogen. Evaporators and gas inlet systems are mounted at the main chamber enabling the observation of growth or adsorption. In the preparation chamber sputtering and annealing of the sample is typically performed. Lastly, the preparation chamber offers the possibility to conduct Auger Electron Spectroscopy (AES) and Residual Gas Analysis (RGA).

\section{Image corrections}

Images are recorded at the screen of the LEEM which consists of microchannel plates, a fluorescent screen, and a CCD-camera. Some microchannel plates differ in thickness from other microchannel plates producing differences in amplification factors. Consequently, contrast gradients occur in the real space images. If the horizontal position of a pixel is given by $x$ and the vertical position of a pixel 


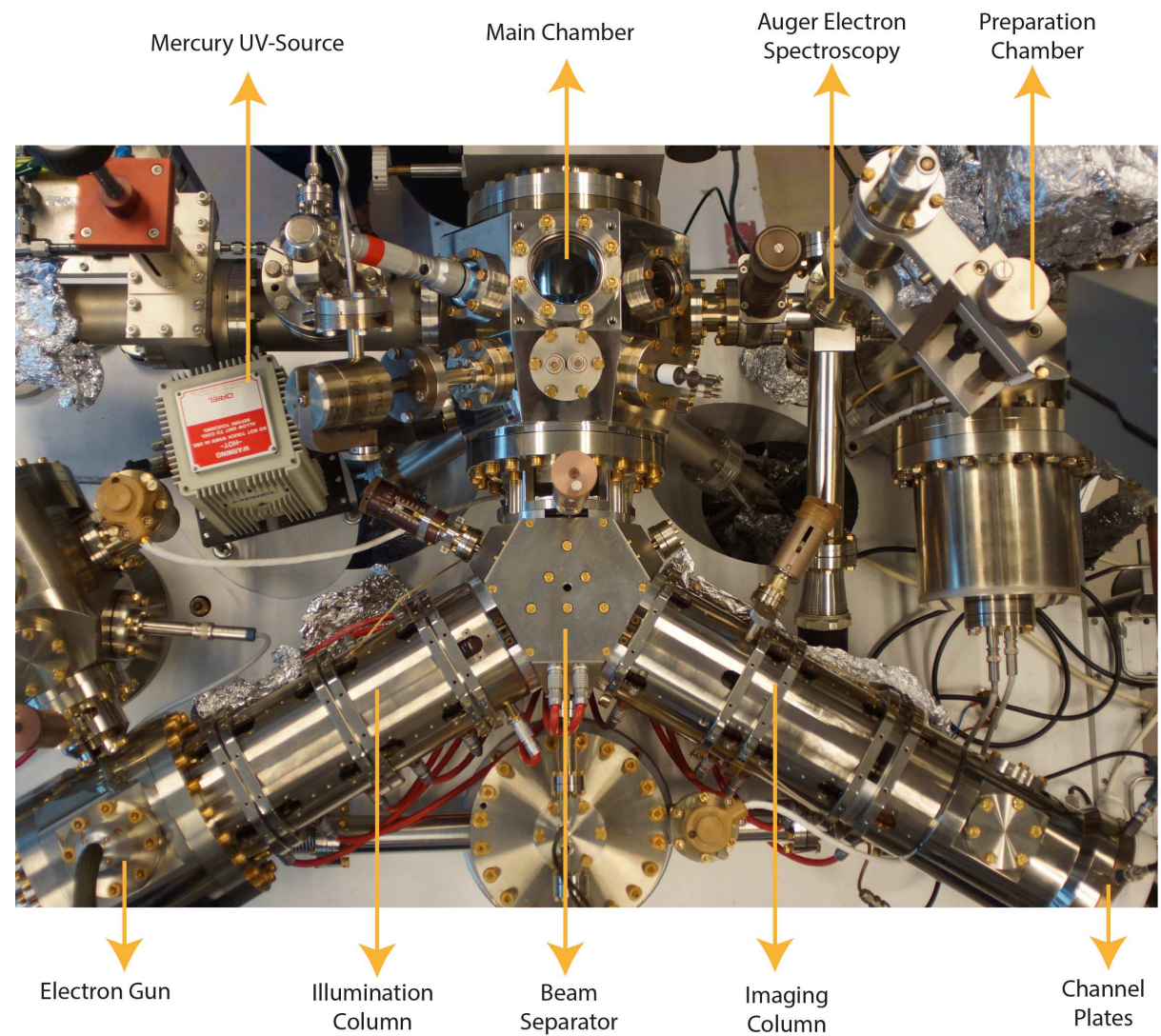

Figure 1.3 - A bird-view photography of the Elmitec LEEM III setup. The arrows indicate important features in the setup. The CCD-camera lies beyond the channel plates and is out of the field of view of the photograph.

by $y$, then the image intensity of a pixel is $\operatorname{Im}(x, y)$. This is the intensity that is recorded after an amplification $A(x, y)$ is factorized at the given pixel. Thus the unamplified intensity of a pixel is $F(x, y)=\operatorname{Im}(x, y) / A(x, y)$. The image intensity of a featureless image recorded in mirror mode, i.e. electron energy of $0 \mathrm{eV}$ or lower, is equal to $A(x, y)$. This mirror image is used as a reference image and the image intensities of the real space images that are to be corrected, are divided by the intensity of the mirror image. An example of the image correction is shown in Fig. 1.4. One can observe that the intensity gradient has diminished and even the microchannel plate defect (the black area at the bottom of the image) is less prominently present as well.

\section{Data analysis}

Real space images offer physical and chemical information which is extracted by different analysis approaches. Area, time, pixel intensity, distance, temperature 

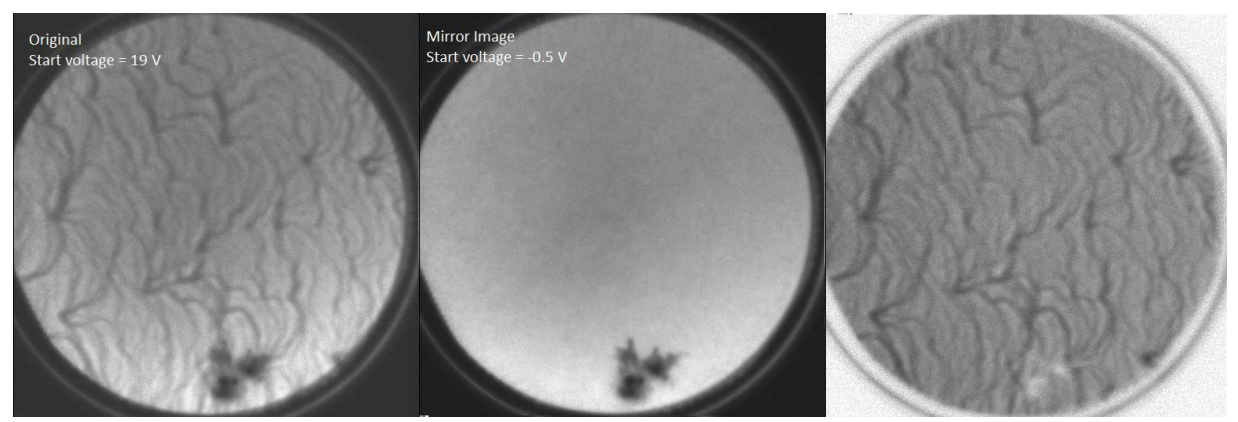

Figure 1.4 - The left panel shows the original image and the middle panel is representing the mirror image. By dividing the original image by the mirror image the correction is fulfilled as can be seen in the right panel. Inhomogeneity across the sample is reduced substantially and the channel plate defect ('dark butterfly' on the bottom) is largely removed as well.

and electron energy are the quantities that are stored in typical low energy electron microscope experiments. The ImageJ software package is used to analyze data.

\section{$\mu$ LEED experiments}

\section{Basics}

Diffracted electrons leaving the sample are focused on the back-focal plane of the objective, forming a diffraction pattern. This makes it possible to relate real space images recorded in LEEM mode to crystal structure information acquired in LEED mode. An even more advantageous feature is the insertion of an illumination aperture that reduces the diameter of the incident electron beam diameter (ultimately down to $1.4 \mu \mathrm{m}$ ), hence the name $\mu$ LEED. A great advantage of inserting such an illumination aperture is that, rather than averaging the LEED patterns of many different structures, one measures only on the desired area of the surface with one or two crystalline domains, which directly yields information on that region's crystal structure. LEED patterns were simulated using the LEEDPat software package [?].

\section{Cumulative $\mu$ LEED experiments}

$\mu$ LEED has been used to determine the superstructures. When recording diffraction patterns at one certain start voltage (or electron energy), not all spots are 
visible simultaneously which causes difficulties in analyzing the diffraction pattern and establishing the symmetry of the superstructures. This was dealed with recording LEED patterns at several start voltages and by summing them into one single image. A drawback of this approach is that the information in the intensities of the spots is lost and, consequently, a structure factor determination of the superstructures becomes impossible from such a cumulative $\mu$ LEED image.

\section{Corrections to reciprocal space images}

Most of the diffraction patterns contain backscattered electrons as well as secondary electrons. Secondary electrons are undesired in reciprocal space images. At first the image is duplicated and its background is subtracted. Minimum, maximum and Gaussian filters are then applied on the subtracted background image. Finally, the original image is divided by the filtered subtracted background image. After adjusting the contrast a corrected LEED pattern looks like what is shown in Fig. 1.5. Inverting pixel intensities was only performed when it made the information in the image better visible.

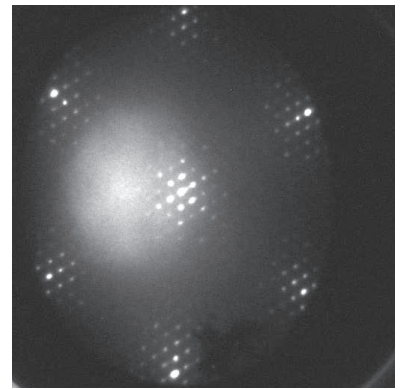

Original

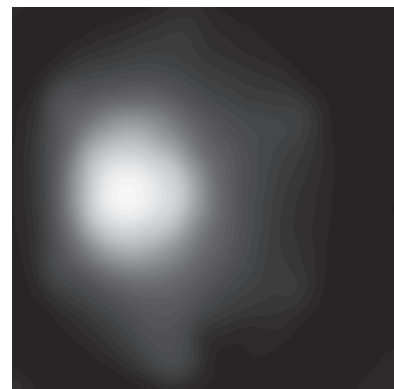

Filtered Background

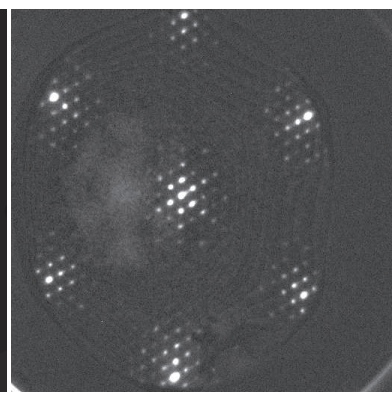

Corrected Image

Figure 1.5 - The left panel shows the original image and the middle panel is the subtracted background that underwent several filters. By dividing the original image by the background the correction is acquired as seen in the right panel. 


\subsubsection{Scanning Tunneling Microscopy}

STM (Scanning Tunneling Microscopy) is a scanning probe technique based on quantum tunneling. The invention of this technique is awarded with the Nobel Prize of Physics in 1986 [?,?]. STM is used to map surface topography in a spatial range from atomic resolution to several micrometers. Furthermore, spectroscopy can be performed in order to determine the local density of states (LDOS).

The basic experimental setup of STM consists of a tip and a sample separated from each other by a distance $z$ as illustrated in Fig. 1.6. The system may be regarded as a quantum mechanical finite barrier in which there is wave propagation from the tip to the sample, while an exponential decay is present in the barrier due to the potential being much higher than the electron energy. In STM it is not the wavefunction $\psi$ that is directly measured, but rather the probability density $|\psi|^{2}$. The tunneling current is proportional to the probability density and is given by:

$$
I \propto \sum_{E_{F}-e V}^{E_{F}}|\psi(0)|^{2} e^{-2 \kappa z} \quad \text { with } \quad \kappa=\frac{\sqrt{m_{e}\left(\phi_{s}+\phi_{t}\right)}}{\hbar}
$$

The probability density starts to decay at $x=0$ by a factor of $e^{-2 \kappa z}$. The factor $\kappa$, usually referred to as the inverse decay length, can be found by solving the Schrödinger equation in the context of Fig. 1.6. The quantities $\phi_{s}, \phi_{t}$ and $m_{e}$ represent the work function of the sample, work function of the tip and the (effective) electron mass respectively. The electrons with energies between $E_{F}$ and $E_{F}-e V$ contribute to the tunneling current, where $E_{F}$ is the Fermi energy and $V$ is the applied potential. By applying a potential between the tip and the sample electrons tunnel from occupied states on one side to unoccupied states on the other side. The direction of the tunnel current is determined by the bias sign. The tunnel current can also be related to the potential by using the Wentzel-Kramer-Brillouin approximation [?] as follows:

$$
I \propto \frac{V}{z} e^{-2 \kappa z}
$$

Typically, STM is operated in either the constant height mode or the constant current mode. In the constant height mode voltage on the z-piezo is fixed, keeping the tip at a constant height, while the current $I$ is recorded. Vice versa, in case of constant current mode the current $I$ is fixed, while the height of the z-piezo is recorded Two types of images are then acquired, i.e. topography maps and current maps.

Furthermore, the STM makes it possible to obtain information about the electronic structure of the sample. This modus operandi is called Scanning Tunneling 


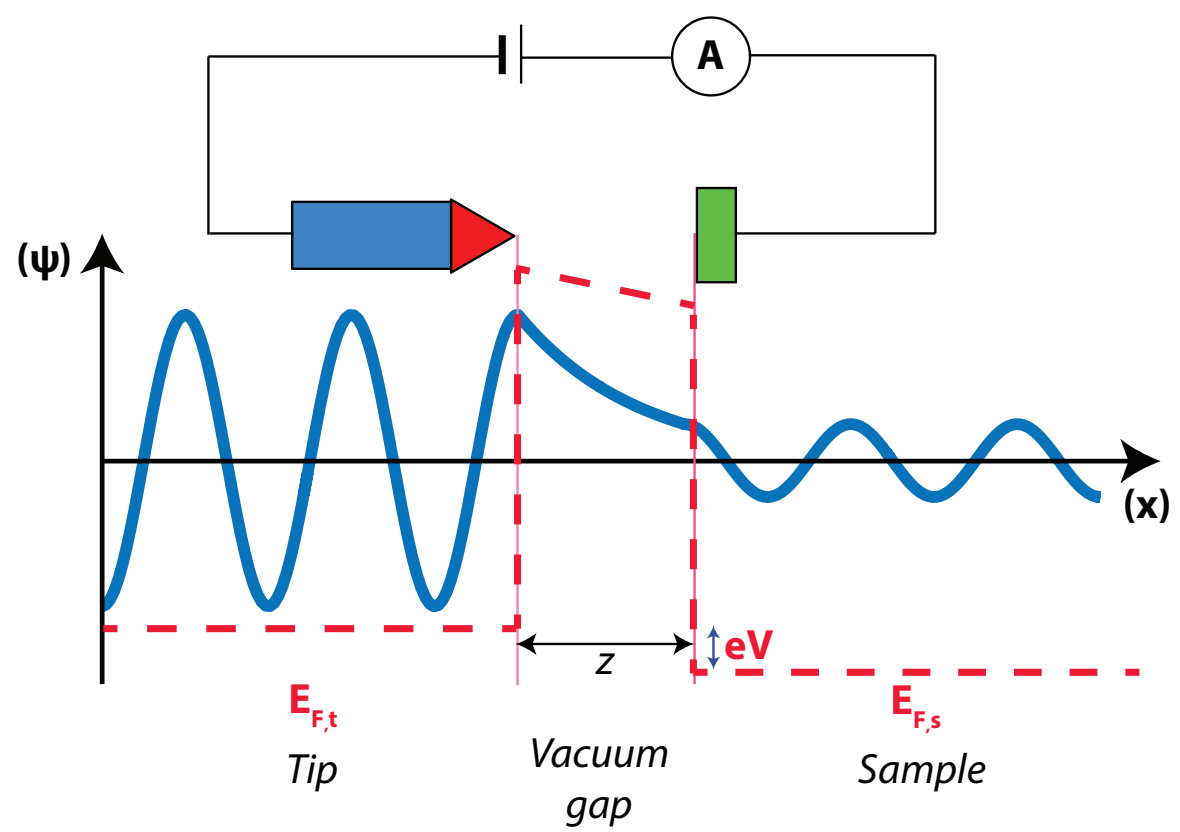

Figure 1.6 - An incident wave from the tip to the vacuum gap changes its characteristics from a periodic wave to an exponentially decaying curve. On the other interface the decayed curve then transforms into a transmitted periodic wave. At the interfaces the wave function and the first derivative of the wave functions must be continuous. In the vacuum gap the potential is higher than the electron energy and thus no periodic wave exists in that regime. A bias voltage is applied across the tip and the sample shifting the

Fermi-levels relatively and thus contributing to a flow of electrons.

Spectroscopy (STS). In STS the tip is held at a given $(x, y)$ position while the feedback loop is switched off. The current is then measured by varying time, height or voltage. For example, in current-time traces dynamics of a system can be investigated. Current versus distance curves give information about the work function of the given sample on location $(x, y)$. Lastly, current-voltage spectroscopy extracts a part of the electronic structure and is discussed below.

\subsubsection{Theory of STS}

In this subsection an expression for the current-voltage characteristics is derived so that the differential conductivity can be obtained and linked to the density of states. Inelastic tunneling is excluded from the derivation. Using Bardeen's approach [?] the tunnel current is given by

$$
I=-\frac{4 \pi q}{\hbar} \int_{\infty}^{\infty}\left|M_{s t}\right|^{2} \rho_{s}\left(\epsilon_{s}\right) \rho_{t}\left(\epsilon_{t}\right) \delta\left(\epsilon_{s}-\epsilon_{t}\right) \mathrm{d} \epsilon
$$


Here, $\left|M_{s t}\right|^{2}$ is the matrix element of the transition probability and incorporates the distance $z$. The factors $\rho_{s}\left(\epsilon_{s}\right)$ and $\rho_{t}\left(\epsilon_{t}\right)$ give the density of states at a specific energy in the sample and the tip respectively. The assumption that only elastic tunneling contributes is done via $\delta\left(\epsilon_{s}-\epsilon_{t}\right)$.

Equation 1.1 is complicated and it is possible to narrow down the equation by implementing approximations. Accurate STS measurements are usually performed at cryogenic temperatures and therefore it is assumed that the Fermi-Dirac distribution is regarded as a step-function. Furthermore, the integral only needs to be taken from $\epsilon_{F}-q V$ to $\epsilon_{F}$. Consequently $\epsilon_{s}=\epsilon$ and $\epsilon_{t}=\epsilon+q V$ is used with the additional implementation of the Fermi-energy $\epsilon_{F}$ set equal to zero. The density of states of the tip is rather flat since the tips are made of either tungsten or an alloy of platinum and iridium. As a result, the density of states of the tip is rewritten as $\rho_{t}(\epsilon+q V) \approx \rho_{t}(0)$. Finally, the assumption that the wave functions of the tip and the sample exhibit minimum overlap resulting in a constant matrix element $\left|M_{s t}\right|^{2}$. The tunneling current is then simplified to

$$
I \approx I_{0} \int_{-q V}^{0} \rho_{s}(\epsilon) \mathrm{d} \epsilon \text { with } I_{0}=-\frac{4 \pi q}{\hbar} \rho_{t}(0)\left|M_{s t}\right|^{2}
$$

The differential conductivity is obtained by taking the derivative of the equation above with respect to the voltage:

$$
\frac{d I}{d V} \propto \rho_{s}(-q V)
$$

\subsubsection{Practical setup and Sample Preparation}

An Omicron low-temperature STM was used to study germanene. A picture of the LT-STM is given in Fig. 1.7. The LT-STM consists of two departments, e.g. the preparation chamber (1) and the main chamber (2). In the preparation chamber sample preparation takes place by sputtering, annealing or deposition. The actual STM is located in a cryostat in the main chamber. The LT-STM is operated under ultrahigh-vacuum conditions. The Ge(110)-samples were cleaned by cycles of $\mathrm{Ar}^{+}$-ion sputtering and annealing $(\mathrm{T}=1100 \mathrm{~K})$ cycles. Platinum deposition was performed by resistive heating of $\mathrm{Pt}$ wrapped around a tungsten wire. During the deposition the Ge(110)-sample was kept at room temperature. Approximately three monolayers of $\mathrm{Pt}$ were deposited on the substrate. Subsequently, the sample was annealed to $1100 \mathrm{~K}$ and cooled down slowly. Upon cooling down and below the eutectic temperature, germanene terminated $\mathrm{Ge}_{2} \mathrm{Pt}$ crystals are retrieved [?]. 


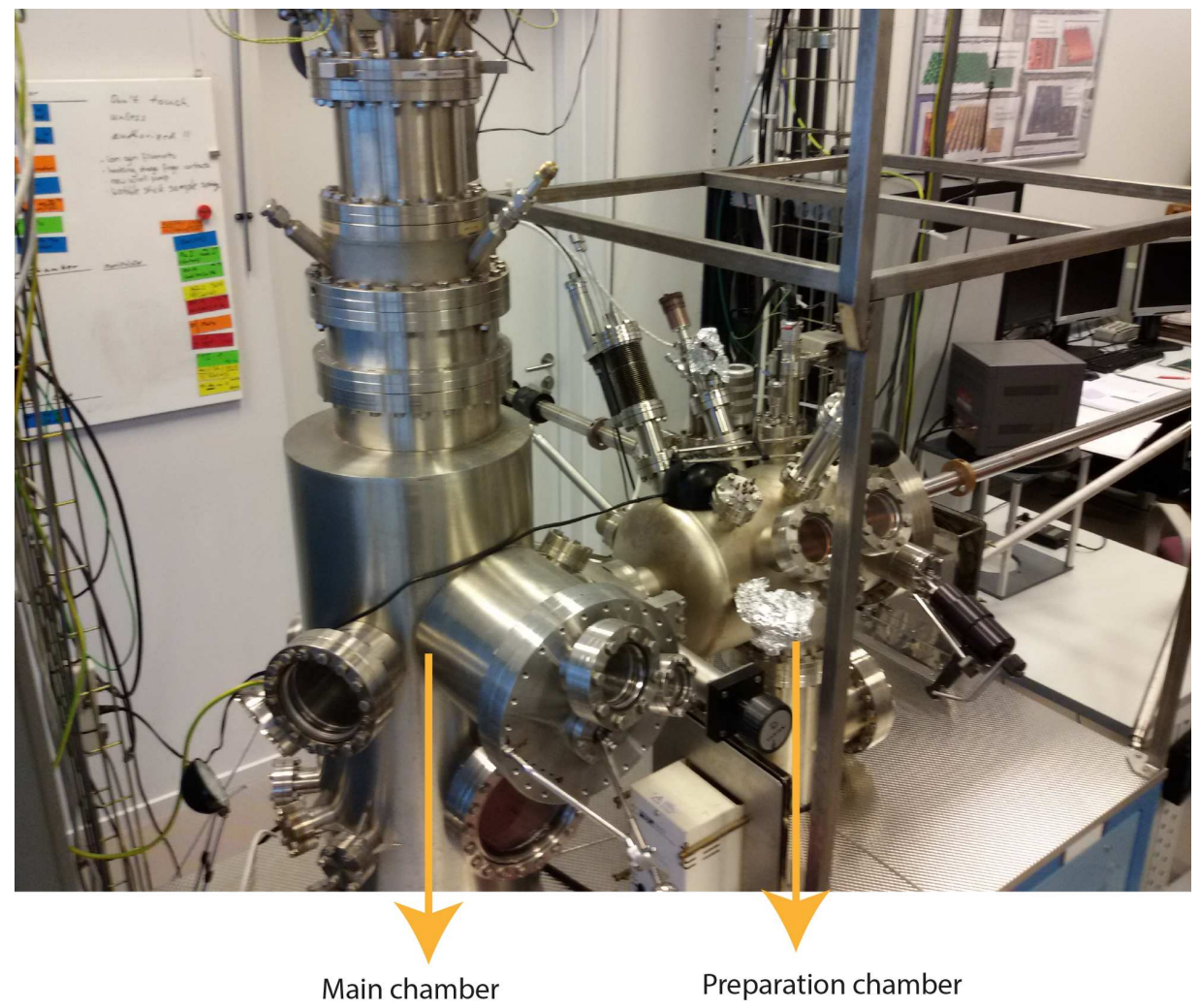

Figure 1.7 - A photograph of the Omicron LT-STM setup. The scanner head is located in the main chamber that is enclosed by a a cryostat. The preparation chamber consists of evaporators, a sputter gun and a sample heating stage. 


\section{Bibliography}

[1] K.S. Novoselov, A.K. Geim, S.V. Morozov, D. Jiang, Y. Zhang, S.V. Dubonos, I.V. Grigorieva, and R.E. Smalley. Science, 306:666-669, 2004.

[2] M. Xu, T. Liang, S. Minmin, and H. Chen. Chem. Rev., 113:3766-3798, 2013.

[3] T.O. Wehling, A.M. Black-Schaffer, and A.V. Balatsky. Adv. Phys., 76(1), 2014.

[4] C.L. Kane and E.J. Mele. Phys. Rev. Lett., 95(146802), 2005.

[5] Y. Zhang, Y.-W. Tan, H.L. Stormer, and P. Kim. Nature, 438:201-204, 2005.

[6] A.H. Castro Neto, F. Guinea, N.M.R. Peres, K.S. Novoselov, and A.K. Geim. Rev. Mod. Phys., 81:109-154, 2009.

[7] J. Zhao, H. Liu, Z. Yu, R. Quhe, S. Zhou, Y. Wang, C.C. Liu, H. Zhong, N. Han, J. Lu, Y. Yao, and K. Wu. Progress in Materials Science, 83:24-151, 2016.

[8] A. Acun, L. Zhang, P. Bampoulis, M. Farmanbar, A. van Houselt, A.N. Rudenko, M. Lingenfelder, G. Brocks, B. Poelsema, M.I. Katsnelson, and H.J.W. Zandvliet. Journal of Physics: Condensed Matter, 27(44), 2015.

[9] F.-F. Zhu, W.-J. Chen, Y. Xu, C.-L. Gao, D.-D. Guan, C.-H. Liu, D. Qian, S.-C. Zhang, and J.-F. Jia. Nature Materials, 14:1020-1025, 2015.

[10] A. Pakdel, Y. Bando, and D. Golberg. Chem. Soc. Rev., 43(934-959), 2014.

[11] A.V. Kolobov and J. Tominaga. Two-dimensional transition metal dichalcogenides. Springer Series in Materials Science, 2016.

[12] L. Li, Y. Yu, G.J. Ye, Q. Ge, X. Ou, H. Wu, D. Feng, X.H. Chen, and Y. Zhang. Nature Nanotechnology, 9:372-377, 2014.

[13] S.P. Koenig, R.A. Doganov, H. Schmidt, A.H. Castro Neto, and volume = 104 pages $=103106$ year $=2014$ B. Özyilmaz, journal $=$ Appl. Phys. Lett.

[14] H. Liu, A.T. Neal, Z. Zhu, Z. Luo, X. Xy, D. Tománek, and P.D. Ye. ACS Nano, 8:4, 2014. 
[15] C. Kamal, A. Chakrabati, and M. Ezawa. New J. of Phys., 17(083014), 2015.

[16] J. Yuan, N. Yu, K. Xue, and X. Miao. Applied Surface Science, 409(85-90), 2017.

[17] E. Aktürk, O.Ü. Aktürk, and S. Ciraci. Phys. Rev. B., 94:014115, 2016.

[18] A.J. Mannix, X.-F. Zhou, B. Kiraly, J.D. Wood, D. Alducin, B.D. Myers, X. Liu, B.L. Fisher, U. Santiago, J.R. Guest, M.J. Yacaman, A. Ponce, A.R. Oganov, M.C. Hersam, and N.P. Guisinger. Science, 350(6267):1513-1516, 2015.

[19] B. Feng, O. Sugino, R.-Y. Liu, J. Zhang, R. Yukawa, M. Kawamura, T. Iimori, H. Kim, Y. Hasegawa, H. Li, L. Chen, K. Wu, H. Kumigashira, F. Komori, T.-C. Chiang, S. Meng, and I. Matsuda. Phys. Rev. Lett., 118:096401, 2017.

[20] B. Feng, J. Zhang, Q. Zhong, W. Li, S. Li, H. Li, P. Cheng, S. Meng, L. Chen, and K. Wu. Nature Chemistry, 8:6, 2016.

[21] Z. Zhang, A.J. Mannix, Z. Hu, B. Kiraly, N.P. Guisinger, M.C. Hersam, and B.I. Yakobson. Nanolett., 16(10):6622-6627, 2016.

[22] K. Takeda and K. Shiraishi. Phys. Rev. B., 50(14916-14922), 1994.

[23] M. Ye, R. Quhe, J. Zheng, Z. Ni, Y. Wang, Y. Yuan, G. Tse, J. Shi, Z. Gao, and J. Lu. Physica E, 59(60), 2014.

[24] M.I. Katsnelson and A. Fasolino. Acc. of Chem. Res., 46(97), 2013.

[25] S. Abdelouahed, A. Ernst, J. Henk, I.V. Maznichenko, and I. Mertig. Phys. Rev. B., 82:125424, 2010.

[26] I. Meric C. Lee L. Wang S. Sorgenfrei K. Watanabe T. Taniguchi P. Kim K.L. Shepard C.R. Dean, A.F. Young and J. Hone. Nature Nanotechnology, 5:722, 2010.

[27] A.F. Young M. Yankowitz B.J. LeRoy K. Watanabe T. Taniguchi P. Moon M. Koshino P. Jarillo-Herrere B. Hunt, J.D. Sanchez-Yamagishi and R.C. Ashoori. Science, 340:1427, 2013.

[28] X. Cui N. Petrone C-H. Lee M.S. Choi D-Y. Lee C. Lee W.J. Yoo K. Watanabe T. Taniguchi C. Nuckolls P. Kim G-H. Lee, Y-J. Yu and J. Hone. ACS Nano, 7(9):7931-7936, 2013.

[29] E. Bauer. Ultramicroscopy, 17:51-56, 1985.

[30] W. Telieps and E. Bauer. Ultramicroscopy, 17:57-65, 1985.

[31] E. Bauer. Reg. Prog. Phys., 57:895-906, 1994.

[32] M.S. Altman. J. Phys.: Condensed Matter, 22:084017, 2010.

[33] K. Hermann and M.A. van Hove. 
[34] G. Binnig, H. Rohrer, Ch. Gerber, and E. Weibel. Appl. Phys. Lett., 40(178), 1982.

[35] G. Binnig and H. Rohrer. IBM Journal of Research and Development, 30(4):35569, 1986.

[36] J.G. Simmons. Journal of Applied Physics, 34(6):1793-1803, 1963.

[37] J. Bardeen. Phys. Rev. Lett., 6(57), 1961.

[38] P. Bampoulis, L. Zhang, A. Safaei, R. van Gastel, B. Poelsema, and H.J.W. Zandvliet. J. Phys.: Condensed Matter, 26:442001, 2014. 



\section{Chapter 2}

\section{Tight-binding method: applied on graphene}

\subsection{Introduction}

In solid state physics the electronic properties of a material are studied in relation to its structure. Various ways of retrieving properties of solids are available, with varying complexity, accuracy and underlying physical approximations. An easy and analytical way of retrieving the electronic structure is via the tight-binding method. In the tight-binding method the weak overlap between atomic (and molecular) orbitals is considered. A wave function is written as a Bloch function that consists of atomic orbitals. The overlap between different atomic orbitals on different sites is calculated and by doing so the dispersion relation of materials can be found. In this chapter the goal is to build a fundament for understanding the electronic structure of the materials of our interest. It is also meant as a guideline for future $\mathrm{PhD}$ and master students to get familiarized with the models.

This chapter consists of an introduction to the crystal structure of the materials of our interest. Then the tight-binding method is explained in detail and applied on graphene by using the first quantization framework. The tight-binding method for graphene is also performed via the second quantization and is given in the Appendix. Again, for graphene the Dirac cone, an exotic feature in the band diagram, is exploited by the long wavelength limit approximation. From these results the density of states per unit area is evaluated and is proved to have linear characteristic. A new Hamiltonian is then constructed for the Dirac fermions by addressing the Fermi-Dirac equation. The band structure of two different edges of graphene is addressed, namely that of armchair and zigzag edges. Last but 
not least, the tight-binding method is briefly applied on hexagonal boron-nitride.

\subsection{Crystal structure}

Graphene, silicene, germanene and hexagonal boron-nitride consist of a single layer of hexagonally ordered atoms. Single layer materials are considered to be two-dimensional, even though an atom is still a three-dimensional object. One reason for these materials to be called two-dimensional arises from the electronic properties that are observed and predicted that fit well in two-dimensional electronic structure models. In this part of the introduction the (origin of the) structures is discussed by kicking off with graphene.

\subsubsection{Crystal structure of graphene}

Pure solid carbon exists in nature in two crystal structures, i.e. diamond and graphite, depending on the hybridization of carbon. The electronic configuration of carbon is [He] $2 \mathrm{~s}^{2} 2 \mathrm{p}^{2}$. Figure 2.1 shows a scheme of how the hybridizations are formed. The excited state of carbon is unstable and tends to decay in one of the two hybridized states. The $s p^{3}$-hybridized state has four equal $s p^{3}$-orbitals establishing a tetrahedral geometry, which results in the diamond crystal structure as given in Fig. 2.2a. The energetically more favorable hybridized state is $s p^{2}$-hybridized consisting of one $p_{z}$-orbital and three $s p^{2}$-orbitals as shown in Fig. 2.2b. The three $s p^{2}$-orbitals are located on the $x y$-plane and are separated by an angle of $120^{\circ}$ from each other forming an hexagonal plane, whereas the $p_{z}$-orbital sticks out of plane. The hexagonal planes are then stacked via the out-of-plane $p_{z}$-orbitals retrieving the crystal structure of graphite as shown in Fig. 2.3. Graphene is the planar result of stripping off one layer of graphite as shown in Fig. 2.4.

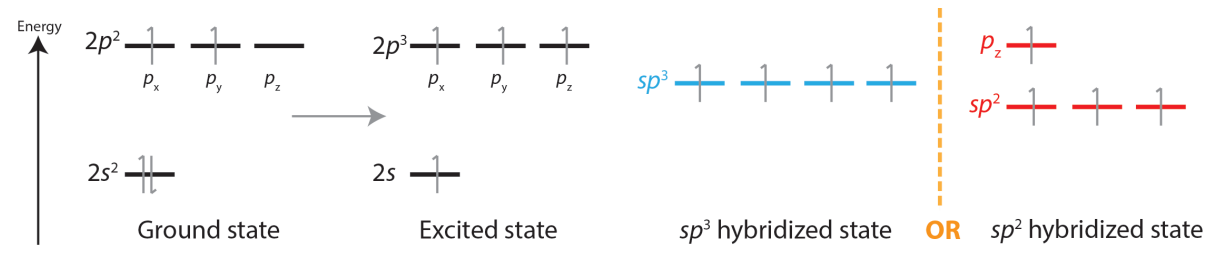

Figure 2.1 - The electrons in the $2 s$ and in the $2 p$-shells are valence electrons. Conventionally one would fill up the valence band as in the ground state. An electron from the $2 s$-shell excites to a $2 p$-shell and thus carbon is in an excited unstable state. Upon lowering the total energy of the system two hybridizations become possible. The $s p^{3}$ hybridization has four equal orbitals. The second possibility is the $s p^{2}$-hybridization with three equal $s p^{2}$-orbitals and one $p_{z}$-orbital. 

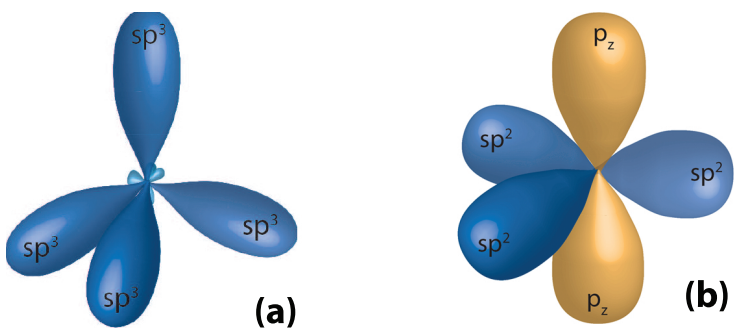

Figure 2.2 - Four $s p^{3}$-orbitals are equally separated from each other in three dimensions resulting in tetrahedral geometry (a). Three $s p^{2}$-orbitals are distributed in a two-dimensional plane where each of them is separated from each other by an angle of $120^{\circ}$. Perpendicular to this plane a $p_{z}$-orbital is located (b).

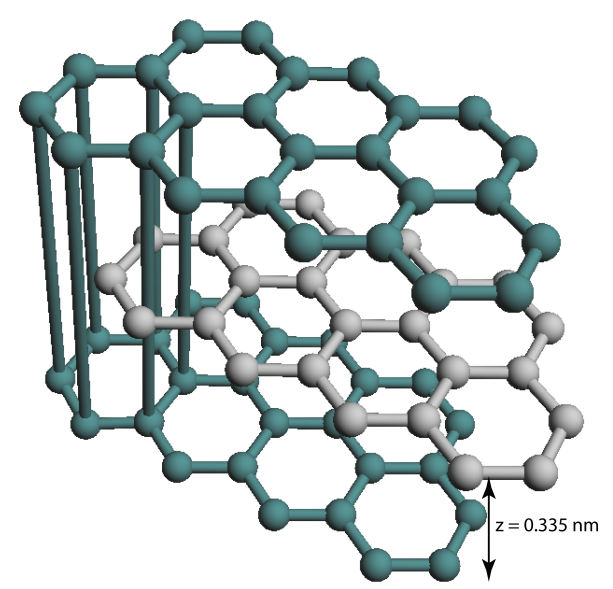

Figure 2.3 - Graphite is a $s p^{2}$-hybridized state of carbon. In-plane the hexagonal structure is retrieved due to a $120^{\circ}$ angle provided by the three $s p^{2}$-orbitals. Perpendicular to this plane the $p_{z}$-orbitals bond with each other, thus graphite is a stacking of graphene layers.

For graphene the neighboring carbon atoms distance is $1.42 \AA$ and the lattice parameter amounts to be $2.46 \AA$ [?]. This small atom-to-atom distance causes the $p_{z}$-orbitals of neighboring carbon atoms to overlap with each other and thereby form $\pi$-bonds. The latter is allowed for graphene since the $p_{z}$-orbital is originally not bonded and half-filled with only one electron. In an hexagonal structure the $\pi$-bonds smear out and the $p_{z}$-electrons become delocalized in graphene. Furthermore, as the $\pi$-bonding is dominant over $\sigma$-bondings in graphene, the coupling between the $p_{z}$-electrons and the nucleus becomes insignificant [?]. As a result, the $p_{z}$-electrons are considered to be independent electrons. Another type of bonding is found in the in-plane structure of graphene, where between 


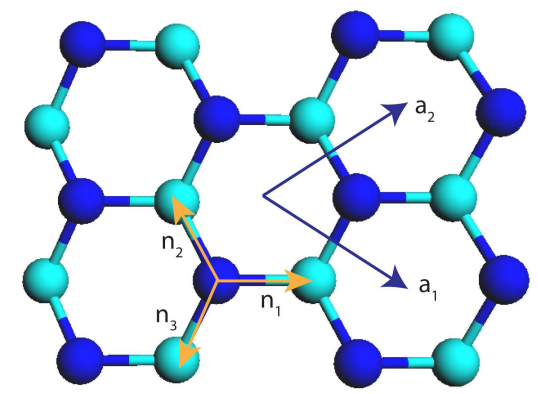

Top view

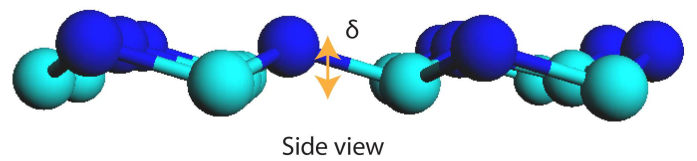

Figure 2.4 - The $s p^{2}$ and $s p^{2}$-like hybridized materials have two sub-lattices as indicated with two different colours. The purely $s p^{2}$-hybridized materials have no buckling and are entirely flat. Examples of these materials are graphene and hexagonal boron-nitride. Their counterparts are silicene and germanene and possess slight buckling.

two neighboring carbon atoms, there is a $\sigma$-bonding. Nuclei of the carbon atoms interact stronger with $\sigma$-electrons than they interact with $\pi$-electrons since the $\sigma$-electrons overlap more with the (Coulomb) potential generated by the nuclei than the $\pi$-electrons. Electrons in $\sigma$-bonds do not contribute to electronic transport since they are filled and hence it can be assumed that all electronic properties are derived from the $p_{z}$-orbitals. This can be seen from the band structures as retrieved from Density Functional Theory (DFT) calculations where the $\pi$-bonding only overlaps insignificantly at high energies (above $3 \mathrm{eV}$ ) with $\sigma$-bondings [?].

An approximation of the electronic band structure of graphene can be calculated by the tight-binding model [?]. The method consists of superposing wave functions for isolated atoms. Therefore, the location of the atomic sites is of utmost importance for the tight-binding model calculations. Let us then describe the crystal structure of graphene in both real space as in reciprocal space. Figure 2.4 shows the real space crystal structure of the materials of our interest. In case of graphene the buckling is zero and the structure is completely flat. The lattice base vectors $\overrightarrow{a_{1}}$ and $\overrightarrow{a_{2}}$ form the unit cell of graphene and are given by:

$$
\mathbf{a}_{\mathbf{1}}=a\left(\begin{array}{c}
\frac{1}{2} \sqrt{3} \\
-\frac{1}{2}
\end{array}\right)\left(\begin{array}{c}
\hat{\mathbf{x}} \\
\hat{\mathbf{y}}
\end{array}\right) \text { and } \mathbf{a}_{\mathbf{2}}=a\left(\begin{array}{c}
\frac{1}{2} \sqrt{3} \\
\frac{1}{2}
\end{array}\right)\left(\begin{array}{c}
\hat{\mathbf{x}} \\
\hat{\mathbf{y}}
\end{array}\right)
$$

For the sake of convenience the normal vectors are further skipped unless re- 
quired. The magnitude of both base vectors are equivalent ( $a=2.46 \AA$ ). The neighboring atom-atom distance is $1.42 \AA$. Each atom is surrounded by three neighbouring atoms as illustrated in Fig. 2.4. Their corresponding vectors are

$$
\mathbf{n}_{\mathbf{1}}=a\left(\frac{1}{\sqrt{3}}, 0\right) \text { and } \mathbf{n}_{2}=a\left(\frac{-1}{2 \sqrt{3}}, \frac{1}{2}\right) \text { and } \mathbf{n}_{3}=a\left(\frac{-1}{2 \sqrt{3}}, \frac{-1}{2}\right)
$$

By using the two-dimensional variant of the definition of the reciprocal lattice $\mathbf{b}_{\mathbf{i}} \cdot \mathbf{a}_{\mathbf{j}}=2 \pi \delta_{i j}$, where $\delta_{i j}$ is the Kronicker-delta function, a set of four equations with four variables is found.

$$
\begin{aligned}
& \text { i } b_{1 x} a_{1 x}+b_{1 y} a_{1 y}=\frac{1}{2} a \sqrt{3} b_{1 x}-\frac{1}{2} a b_{1 y}=2 \pi \\
& \text { ii } b_{2 x} a_{1 x}+b_{2 y} a_{1 y}=\frac{1}{2} a \sqrt{3} b_{2 x}-\frac{1}{2} a b_{2 y}=0 \\
& \text { iii } b_{1 x} a_{1 x}+b_{1 y} a_{1 y}=\frac{1}{2} a \sqrt{3} b_{2 x}+\frac{1}{2} a b_{2 y}=0 \\
& \text { iv } b_{2 x} a_{1 x}+b_{2 y} a_{1 y}=\frac{1}{2} a \sqrt{3} b_{2 x}+\frac{1}{2} a b_{2 y}=2 \pi
\end{aligned}
$$

The unit cell in reciprocal space is constructed by the solutions of the aforementioned set of equations. The solutions, i.e. the reciprocal lattice base vectors, are given in Eq. 2.1 and the reciprocal space is constructed accordingly as shown in Fig. 2.5.

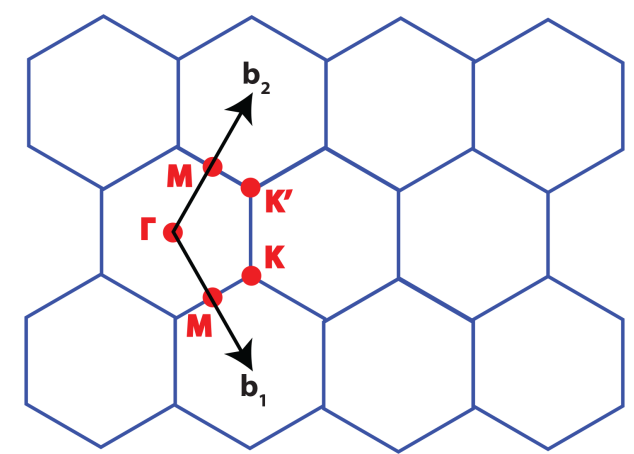

Figure 2.5 - The reciprocal space lattice of graphene provides a hexagonal lattice structure with four special points (i.e. $\Gamma, \mathrm{K}, \mathrm{K}^{\prime}$ and $\mathrm{M}$ )

$$
\mathbf{b}_{1}=\frac{2 \pi}{a}\left(\begin{array}{c}
\frac{1}{\sqrt{3}} \\
-1
\end{array}\right) \quad \text { and } \quad \mathbf{b}_{2}=\frac{2 \pi}{a}\left(\begin{array}{c}
\frac{1}{\sqrt{3}} \\
1
\end{array}\right)
$$

Four special locations are defined on the reciprocal lattice as shown in Fig. 2.5:

$$
\begin{gathered}
\Gamma=\left(\begin{array}{c}
0 \\
0
\end{array}\right)\left(\begin{array}{c}
\hat{\mathbf{x}} \\
\hat{\mathbf{y}}
\end{array}\right) \\
\mathbf{M}=\frac{2 \pi}{a}\left(\begin{array}{c}
\frac{1}{2 \sqrt{3}} \\
\frac{-1}{2}
\end{array}\right)\left(\begin{array}{c}
\hat{\mathbf{x}} \\
\hat{\mathbf{y}}
\end{array}\right) \\
\mathbf{K}=\frac{2 \pi}{a}\left(\begin{array}{c}
\frac{1}{\sqrt{3}} \\
\frac{-1}{3}
\end{array}\right)\left(\begin{array}{c}
\hat{\mathbf{x}} \\
\hat{\mathbf{y}}
\end{array}\right)
\end{gathered}
$$




$$
\mathbf{K}^{\prime}=\frac{2 \pi}{a}\left(\begin{array}{c}
\frac{1}{\sqrt{3}} \\
\frac{1}{3}
\end{array}\right)\left(\begin{array}{c}
\hat{\mathbf{x}} \\
\hat{\mathbf{y}}
\end{array}\right)
$$

All interesting phenomena in graphene and the other two-dimensional materials discussed in this thesis take place at the K-point. The tight-binding models in this chapter are used to elaborate on the properties of graphene.

\subsubsection{Crystal structure of silicene and germanene}

\begin{tabular}{|l|l|l|l|l|l|}
\hline & Sublattice 1 & Sublattice 2 & Lattice Parameter $a(\AA)$ & Buckling $\delta(\AA)$ & Hybridization \\
\hline Graphene & Carbon & Carbon & 2.46 & 0 & $s p^{2}$ \\
\hline Silicene & Silicon & Silicon & 3.95 & 0.44 & $s p^{2} / s p^{3}$ \\
\hline Germanene & Germanium & Germanium & 4.12 & 0.65 & $s p^{2} / s p^{3}$ \\
\hline$h$-BN & Boron & Nitrogen & 2.52 & 0 & $s p^{2}$ \\
\hline
\end{tabular}

The origin of the electron structure of graphene can be traced back to the electronic configuration of carbon atoms. In the periodic table of the elements silicon and germanium are located below carbon meaning that silicon and germanium possess similar electronic configurations to graphene, namely [Ne] $3 s^{2} 3 p^{2}$ and $[\mathrm{Ar}] 4 \mathrm{~s}^{2} 4 \mathrm{p}^{2}$ respectively. Intuitively, one may be inspired to think of twodimensional silicon and germanium layers exhibiting an hexagonal lattice. These materials are called silicene and germanene respectively. Theoretical calculations have shown that these two materials are truly alike and do not differ in essence from each other [?]. Therefore, silicene will be taken as an example to address the origin of the structure.

The structural and electronic properties were calculated thoroughly by Takeda et al. [7] and Cahangirov et al. [?]. In this paragraph a small summary on their conclusions is forwarded. While silicon has the electronic configuration similar to that of graphene, it is unable to form graphitic-like (and graphene-like) sheets of hexagonal silicon layers. Silicon atoms are larger than carbon atoms and larger interatomic distances are found. Consequently, the $p_{z}$-orbitals are separated further away from each other and hence the overlap between the $p_{z}$-orbitals reduces drastically. It is important to note that $\sigma$-bondings take much more space than $\pi$-bondings. In case of a fully planar configuration the overlap between $\sigma$-bondings is much stronger than $\pi$-bondings. DFT (Density Functional Theory) calculations reveal phonon modes for this entirely flat configuration. Thus one can conclude that a flat stucture for silicene is rather impossible. By slightly buckling the in-plane structure, i.e. vertically shifting a sub-lattice relatively to another sub-lattice as depicted in Fig. 2.4, the $\pi$-bondings are restored. The price that is paid is $(\sigma, \pi)$-mixing, which results in reduced dominance of the $p_{z}$-orbitals. Nevertheless, graphene-like electronic properties are conserved. Additional features are found for silicene and germanene. For example, not only is the spin-orbit coupling enhanced by larger atomic numbers of silicon and 
germanium compared to graphene, but also due to the $(\sigma, \pi)$-mixing a stronger spin-orbit coupling is predicted [?]. Furthermore, the possibility of opening a band gap is there for silicene and germanene [?].

The buckling of silicene is caused by the pseudo Jahn-Teller effect [?]. The conventional Jahn-Teller effect states that any non-linear molecular system in a degenerate electronic state will be unstable and will undergo distortion to form a system of lower symmetry and lower energy, thereby removing the degeneracy [?]. Forming a system of lower symmetry and lower energy is done by either compressing or elongating the crystal. An example is given in Fig. 2.6. In the center of the figure the ground state of a (random) material is given. Firstly, assume that the electronic configuration is degenerate. The degeneracy is removed by shifting (some) atomic orbitals in energy. In turn another (lower) symmetry is retrieved. For example, if the energy of the $d_{z^{2}}$ is lowered, then this orbital will be energetically more favourable and hence elongation will take place. The assumption of degenerate electronic configurations does not hold for the two-dimensional materials of our interest. Figure 2.1 illustrates clearly that the $s p^{2}$ and $s p^{3}$-hybridizations of carbon, silicon and germanium are nondegenerate. Applying the conventional Jahn-Teller effect is therefore not valid to explain the buckling of silicene and germanene. Since the electrons have interaction with the nuclei one needs to take into account vibrational states as well. The coupling between electronic and vibrational states is named vibronic coupling. These vibrational states are typically non-symmetrical and reduce therefore the symmetry of the electronic structure. Lower the symmetry by means of vibrational states is called the pseudo Jahn-Teller effect. Graphene has negligible $(\sigma, \pi)$-mixing, i.e. no vibronic coupling, while silicene and germanene exhibit significant mixing of these bondings. Vibronic coupling eventually leads to a lower symmetry by inducing puckering into silicene and germanene [?,?,?].

Following the same arguments as for silicene the structure of germanene is explained. The structure is shown in Fig. 2.4. For germanene the interatomic distance is even greater than that for silicene, thus stronger buckling is required in order to preserve the graphene-like electronic properties. The lattice parameters for silicene and germanene are found to be $2.25 \AA$ and $2.38 \AA$ respectively. The buckling heights of silicene and germanene are calculated to be $0.44 \AA$ and $0.64 \AA ̊$ respectively [?].

\subsubsection{Crystal structure of hexagonal boron-nitride}

Yet another $s p^{2}$-hybridized material is h-BN, or hexagonal boron-nitride. This material is, like graphene, entirely flat. The difference with graphene is that now the two sub-lattices of h-BN are composed of two different elements rather than one single element (e.g. carbon). As the name obviously suggests, h-BN consists 


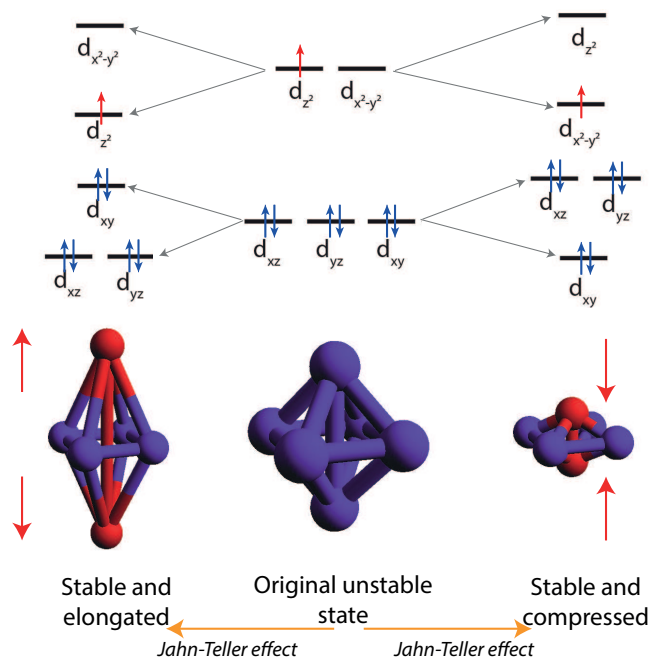

Figure 2.6 - The Jahn-Teller effect distorts degenerate systems and transforms them into non-degenerate systems by forming lower symmetry and therefore lower energy. The distortion leads to deformations in the crystal.

of boron and nitrogen atoms. In the periodic table of the elements boron is the left neighbor of carbon and therefore it contains one electron less in its electronic configuration. The same can be applied to nitrogen, but with the difference that it contains one electron more. The number of electrons does not bother boronnitride to form perfect $s p^{2}$-orbitals which form strong $\sigma$-bondings. However, the striking difference with graphene lies in the $p_{z}$-orbitals of $\mathrm{h}-\mathrm{BN}$ and will be discussed by means of the tight-binding method. One spoiler that can be given is that the electronic properties of h-BN are dramatically different than that of graphene. For example, h-BN is an insulator with a band gap of $5.9 \mathrm{eV}$ [?,?], whereas graphene is a zero band-gap semiconductor [?]. 


\subsection{Tight-binding method: first quantization}

Now define the real space lattice vector $\mathbf{R}$ as $\mathbf{R}=n_{1} \mathbf{a}_{1}+n_{2} \mathbf{a}_{2}$ and allow $\left(n_{1}, n_{2}\right) \in \mathbb{Z}$. This freshly defined vector hops from unit cell to unit cell depending on the values $n_{1}$ and $n_{2}$. Define an analogue to $\mathbf{R}$ in reciprocal space, i.e. $\mathbf{G}=m_{1} \mathbf{b}_{1}+$ $m_{2} \mathbf{b}_{2}$. Furthermore, in reciprocal space any vector is described by $\mathbf{k}=k_{1} \mathbf{b}_{1}+k_{2} \mathbf{b}_{2}$. Unlike $n_{1}, n_{2}, m_{1}$ and $m_{2}$, the factors $k_{1}$ and $k_{2}$ are allowed to take any real number. Again, by using the definition of the reciprocal space $\left(\mathbf{b}_{\mathbf{i}} \cdot \mathbf{a}_{\mathbf{j}}=2 \pi \delta_{i j}\right)$ one finds

$$
\mathbf{k} \cdot \mathbf{R}=2 \pi\left(n_{1} k_{1}+n_{2} k_{2}\right)
$$

and

$$
\mathbf{G} \cdot \mathbf{R}=2 \pi\left(n_{1} m_{1}+n_{2} m_{2}\right)
$$

With $\left(n_{1} m_{1}+n_{2} m_{2}\right)$ always being an integer, the dot product of $\mathbf{G}$ and $\mathbf{R}$ is a multiple of $2 \pi$. Now suppose a vector $\mathbf{k}^{\prime}$ lies outside of the first Brillouin zone. This vector can be described by

$$
\mathbf{k}^{\prime}=\mathbf{k}+\mathbf{G}
$$

It quickly follows that

$$
e^{i \mathbf{k}^{\prime} \cdot \mathbf{R}}=e^{i \mathbf{k} \cdot \mathbf{R}+i \mathbf{G} \cdot \mathbf{R}}=e^{i \mathbf{k} \cdot \mathbf{R}} e^{i \mathbf{G} \cdot \mathbf{R}}=e^{i \mathbf{k} \cdot \mathbf{R}}
$$

since $\mathbf{G} \cdot \mathbf{R}$ gives multiples of $2 \pi$. This means that the solutions acquired within the first Brillouin zone can be generalized throughout the whole lattice. Let us now touch upon the tight-binding method. The tight-binding method uses an approximate set of wave functions for atoms located on atomic sites. In this section only $p_{z}$-orbitals located on the a hexagonal lattice. Calculations beyond these approximations are not the scope of this PhD-thesis. Graphene and hexagonal boron-nitride are used to illustrate the tight-binding method.

The easiest way to calculate the characteristic linear dispersion relation of graphene is by calculating the nearest-neighbor tight-binding methods. Although graphene was actually isolated in 2004, already in the year 1947 the tight-binding description for graphite and graphene was developed by P.R Wallace [?]. In the tightbinding model the $p_{z}$-valence electron has a weak interaction with neighboring ions. In this section the tight-binding model for graphene is shown in detail in order to grab a feeling for other two-dimensional materials.

The tight binding method is a tool to solve the Schrödinger equation by using the Bloch wave theorem. The Schrödinger equation in this context is written as:

$$
\hat{H} \psi_{\mathbf{k}}(\mathbf{r})=E_{\mathbf{k}} \psi_{\mathbf{k}}(\mathbf{r})
$$

The Bloch wave theorem essentially expands a set of atomic orbitals throughout the whole crystal by $\sum_{\mathbf{R}} e^{i \mathbf{k} \cdot \mathbf{R}}$. The Bloch wave function is given as: 


$$
\psi_{\mathbf{k}}(\mathbf{r})=\sum_{\mathbf{R}} e^{i \mathbf{k} \cdot \mathbf{R}} \phi(\mathbf{r}-\mathbf{R}) \text { with the atomic orbitals } \phi(\mathbf{r})=\sum_{n} c_{n} \chi_{n}(\mathbf{r})
$$

Here, $\chi_{n}$ denotes a specific atomic orbital, e.g. $p_{z}$-orbital on sub-lattice $\mathrm{A}$, and $c_{n}$ is its corresponding probability coefficient. Or also written as:

$$
\psi_{\mathbf{k}}(\mathbf{r})=\sum_{\mathbf{R}} \sum_{n} c_{n} e^{i \mathbf{k} \cdot \mathbf{R}} \chi_{n}(\mathbf{r}-\mathbf{R})
$$

What is essentially desired is to find solutions to the Schrödinger equation, the k-dependence of the energy for each orbital are determined. Therefore the Schrödinger equations needs to be multiplied by the orbital wave function.

$$
\left\langle\chi_{m}|\hat{H}| \psi_{\mathbf{k}}\right\rangle=E_{\mathbf{k}}\left\langle\chi_{m} \mid \psi_{\mathbf{k}}\right\rangle
$$

For multiple orbital wave functions the problem leads to two matrices, namely the Hamiltonian and the overlap matrix via.

$$
\sum_{n} H_{m n, \mathbf{k}} c_{n}=E_{\mathbf{k}} \sum_{n} S_{m n, \mathbf{k}} c_{n}
$$

The elements of the Hamiltonian and overlap matrix are given by

$$
\begin{aligned}
H_{m n, \mathbf{k}} & =\sum_{\mathbf{R}} e^{i \mathbf{k} \cdot \mathbf{R}}\left\langle\chi_{m}(\mathbf{r})|\hat{H}| \chi_{n}(\mathbf{r}-\mathbf{R})\right\rangle \\
S_{m n, \mathbf{k}} & =\sum_{\mathbf{R}} e^{i \mathbf{k} \cdot \mathbf{R}}\left\langle\chi_{m}(\mathbf{r}) \mid \chi_{n}(\mathbf{r}-\mathbf{R})\right\rangle
\end{aligned}
$$

Assuming the atomic orbitals are orthogonal, then the elements of the overlap matrix are given by $S_{m n, \mathbf{k}}=\delta_{m n}$. Graphene and hexagonal boron-nitride are assumed to contribute with only its two $p_{z}$-orbitals in a single unit cell. The sublattices are labeled with the indices $A$ and $B$ respectively. The valence orbitals $\chi_{A}$ and $\chi_{B}$ are taken into account and this results in a $(2 \times 2)$-matrix with elements $H_{A A}, H_{A B}, H_{B A}$ and $H_{B B}$. For the element $H_{A A}, \mathbf{R}$ is set zero due to the nearest neighbour approximation. With respect to a specific A-atom the nearest A-atom is located at the next-nearest neighbour distance. Consequently, it is assumed that it does not lead to any contribution to the Hamiltonian within the nearest neighbour approximation. The same holds for $H_{B B}$. The elements $H_{A B}$ and $H_{B A}$ have non-zero contribution in the nearest neighbor approximation in which $\mathrm{R}$ is a set of the three atomic site vectors $\overrightarrow{n_{1}}, \overrightarrow{n_{2}}$ and $\overrightarrow{n_{3}}$. Since there is rotational symmetry in the $p_{z}$ orbitals and the distance of an atom to each of his neighbours is equivalent, the overlap integral can be regarded as a constant $-t$. $H_{B A}$ is just the complex conjugate of $H_{A B}$

$$
\begin{gathered}
H_{A A}=\left\langle\chi_{A}(\mathbf{r})|\hat{H}| \chi_{A}(\mathbf{r})\right\rangle=\epsilon_{A} \\
H_{A B}=\sum_{j=1}^{3} e^{i \mathbf{k} \cdot \mathbf{n}_{\mathbf{j}}}\left\langle\chi_{A}(\mathbf{r})|\hat{H}| \chi_{B}\left(\mathbf{r}-\mathbf{n}_{\mathbf{j}}\right)\right\rangle=-t\left(e^{i \mathbf{k} \cdot \mathbf{n}_{1}}+e^{i \mathbf{k} \cdot \mathbf{n}_{2}}+e^{i \mathbf{k} \cdot \mathbf{n}_{3}}\right)=-t_{k}
\end{gathered}
$$




$$
\begin{gathered}
H_{B A}=\sum_{j=1}^{3} e^{i \mathbf{k} \cdot \mathbf{n}_{\mathbf{j}}}\left\langle\chi_{B}(\mathbf{r})|\hat{H}| \chi_{A}\left(\mathbf{r}-\mathbf{n}_{\mathbf{j}}\right)\right\rangle=-t\left(e^{-i \mathbf{k} \cdot \mathbf{n}_{1}}+e^{-i \mathbf{k} \cdot \mathbf{n}_{2}}+e^{-i \mathbf{k} \cdot \mathbf{n}_{3}}\right)=-t_{k}^{*} \\
H_{B B}=\left\langle\chi_{B}(\mathbf{r})|\hat{H}| \chi_{B}(\mathbf{r})\right\rangle=\epsilon_{B}
\end{gathered}
$$

Here, $t$ is defined as $\left\langle\chi_{A}(\mathbf{r})|\hat{H}| \chi_{B}\left(\mathbf{r}-\mathbf{n}_{\mathbf{j}}\right)\right\rangle$ and is the hopping parameter from atom A to its nearest neighbour atom $B$. The matrix elements define the $(2 \times 2)$-matrix of which the eigenvalues need to be solved to retrieve the dispersion relation:

$$
\begin{gathered}
\left(\begin{array}{cc}
\epsilon_{A} & -t_{\mathbf{k}} \\
-t_{\mathbf{k}}^{*} & \epsilon_{B}
\end{array}\right)\left(\begin{array}{c}
c_{A} \\
c_{B}
\end{array}\right)=E_{\mathbf{k}}\left(\begin{array}{c}
c_{A} \\
c_{B}
\end{array}\right) \\
\left|\begin{array}{cc}
\epsilon_{A}-E_{\mathbf{k}} & -t_{\mathbf{k}} \\
-t_{\mathbf{k}}^{*} & \epsilon_{B}-E_{\mathbf{k}}
\end{array}\right|=0
\end{gathered}
$$

Rewrite as a quadratic function:

$$
E_{\mathbf{k}}^{2}-E_{\mathbf{k}}\left(\epsilon_{A}+\epsilon_{B}\right)+\left(\epsilon_{A} \epsilon_{B}-t_{\mathbf{k}}^{*} t_{\mathbf{k}}\right)=0
$$

Two solution are then found

$$
E_{\mathbf{k}}=\frac{\left(\epsilon_{A}+\epsilon_{B}\right) \pm \sqrt{\left(\epsilon_{A}+\epsilon_{B}\right)^{2}-4\left(\epsilon_{A} \epsilon_{B}-t_{\mathbf{k}}^{*} t_{\mathbf{k}}\right)}}{2}
$$

In case of graphene the sub-lattices comprise of two carbon atoms and requires that $\epsilon_{A}=\epsilon_{B}$. Define this valence orbital energy as $E_{p}$. The eigenvalues of the matrix are

$$
E_{\mathbf{k}}=E_{p} \pm \sqrt{t_{\mathbf{k}}^{*} t_{\mathbf{k}}}
$$

with the normalized eigenvectors

$$
\varphi_{1}=\frac{1}{\left|t_{\mathrm{k}}\right| \sqrt{2}}\left(\begin{array}{c}
t_{\mathrm{k}} \\
\left|t_{\mathrm{k}}\right|
\end{array}\right) \text { and } \varphi_{2}=\frac{1}{\left|t_{\mathrm{k}}\right| \sqrt{2}}\left(\begin{array}{c}
-t_{\mathrm{k}} \\
\left|t_{\mathrm{k}}\right|
\end{array}\right)
$$

Now the expansion of previously defined quantities can be started by writing $t_{\mathbf{k}}$ as $t\left(e^{i k \cdot n_{1}}+e^{i k \cdot n_{2}}+e^{i k \cdot n_{3}}\right)$. The exponents are also evaluated accordingly to the neighbouring vectors as given in Sec.2.2.1. Following this route step by step leads to Eq. 2.5.

$$
\begin{gathered}
E_{\mathbf{k}}=E_{p} \pm t \sqrt{\left(e^{i \mathbf{k} \cdot \mathbf{n}_{1}}+e^{i \mathbf{k} \cdot \mathbf{n}_{2}}+e^{i \mathbf{k} \cdot \mathbf{n}_{3}}\right)\left(e^{-i \mathbf{k} \cdot \mathbf{n}_{1}}+e^{-i \mathbf{k} \cdot \mathbf{n}_{2}}+e^{-i \mathbf{k} \cdot \mathbf{n}_{3}}\right)} \\
\Longrightarrow E_{\mathbf{k}}=E_{p} \pm t \sqrt{3+2 \cos \left(\frac{k_{x} a \sqrt{3}}{2}-\frac{k_{y} a}{2}\right)+2 \cos \left(k_{y} a\right)+2 \cos \left(\frac{k_{x} a \sqrt{3}}{2}+\frac{k_{y} a}{2}\right)}
\end{gathered}
$$

$\mathrm{E}_{p}$ is chosen to be $0 \mathrm{eV}$, the hopping parameter $\mathrm{t}$ is $3 \mathrm{eV}$ as derived from DFTcalculations [?] and the lattice parameter is $2.46 \AA$ [?]. With Eq. 2.5 it is now 
possible to plot the band diagram. In order to do so one should select the regions of interest in reciprocal space. For that purpose the four high symmetry locations in reciprocal space as given in Fig. 2.5 are used, which are given by the following eigenvalues and eigenvectors:

$$
\begin{gathered}
E_{\Gamma^{+}}=E_{p}+3 t ; \quad \Gamma^{+}=\frac{1}{\sqrt{2}}\left(\begin{array}{l}
1 \\
1
\end{array}\right) \quad \text { and } E_{\Gamma^{-}}=E_{p}-3 t ; \Gamma^{-}=\frac{1}{\sqrt{2}}\left(\begin{array}{c}
-1 \\
1
\end{array}\right) \\
E_{M^{+}}=E_{p}+t ; M^{+}=\frac{1}{\sqrt{2}}\left(\begin{array}{l}
1 \\
1
\end{array}\right) \text { and } E_{M^{-}}=E_{p}-t ; M^{-}=\frac{1}{\sqrt{2}}\left(\begin{array}{c}
-1 \\
1
\end{array}\right) \\
E_{K^{+}}=E_{p} ; K^{+}=\left(\begin{array}{l}
1 \\
0
\end{array}\right) \text { and } E_{K^{-}}=E_{p} ; K^{-}=\left(\begin{array}{c}
0 \\
1
\end{array}\right) \\
E_{K^{++}}=E_{p} ; K^{\prime+}=\left(\begin{array}{l}
1 \\
0
\end{array}\right) \text { and } E_{K^{\prime-}}=E_{p} ; K^{\prime-}=\left(\begin{array}{l}
0 \\
1
\end{array}\right)
\end{gathered}
$$

The $\mathrm{K}$ and $\mathrm{K}^{\prime}$-points are degenerate and with spin degeneracy the Fermi level should be in the middle of these two bands (i.e. half-filled bands of $K$ and $K^{\prime}$ ). Figure 2.7 shows the final result of the tight-binding method. The band structure diagram shows interesting features in the vicinity of the K-point. The bands do not cross the Fermi-level making graphene a non-metal material. Interestingly, the band gap as a non-metal material is zero in this calculation (excluding spinorbit coupling). Therefore, graphene is a zero band gap semiconductor (or what is also called a semimetal). The second notable feature is the linearity around the $\mathrm{K}$-point from which the famous linear dispersion relation is derived from. Due to its conical geometry around the K-point this feature is called the Dirac cone. Basically, the Dirac cone is just the result of graphene being a two-dimensional hexagonal material whose neighboring (half-filled) $p_{z}$-orbitals have the most dominant bonding (i.e. $\pi$-bonding). The linearity around the K-point is further investigated by using the long wavelength approximation (Sec. 2.4).

\subsection{The long wavelength limit approximation}

Figure 2.7 depicts the band diagram of graphene. Around the Fermi-level, i.e. E $=0$, only the K-point contributes significantly. In this section only the electron near the K-point is taken into consideration. The coordinates of the K-point (and the atomic neighbour vectors) were given in above. Electrons near the Fermi level are described by $\mathbf{q}$, where $|\mathbf{q}| \ll|\mathbf{K}|$ considers only the electrons around the Fermi level. One can analogously apply the same trick for the $\mathrm{K}^{\prime}$-point leading to the same result. Any state in the first Brillouin zone near the Fermi level can be written as

$$
\mathbf{k}=\mathbf{K}+\mathbf{q}
$$




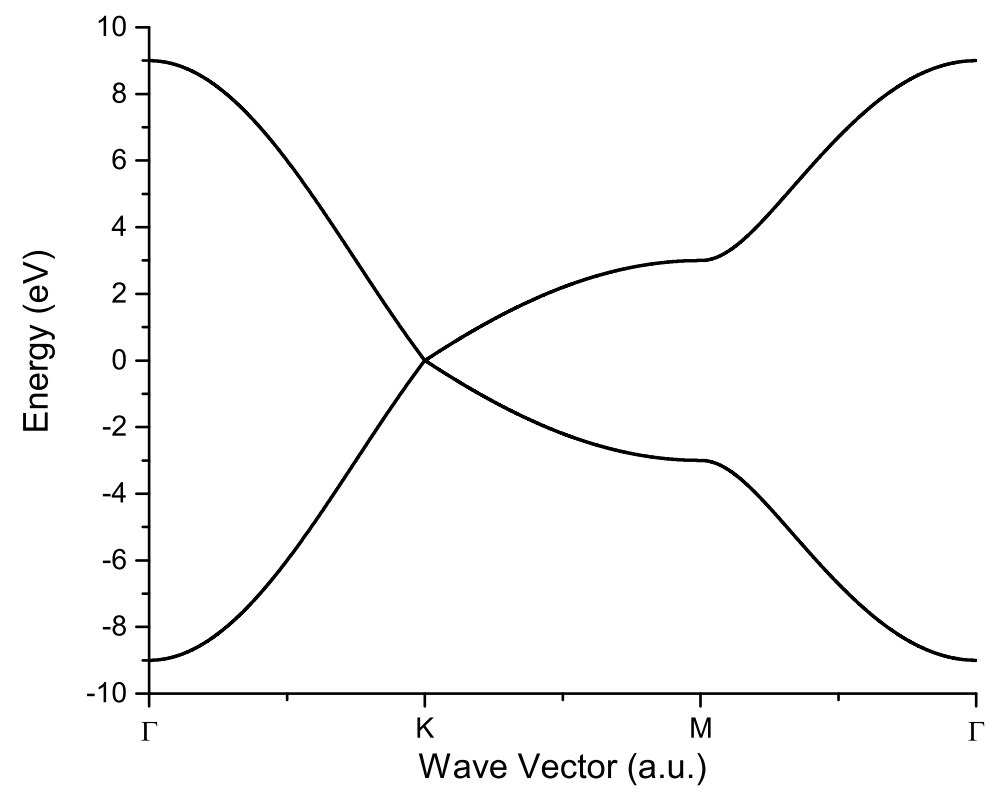

Figure 2.7 - Band structure of graphene via tight-binding. At the K-point the Dirac-cone is visible. The curve above the Fermi energy, that is at $0 \mathrm{eV}$, originates from the plus-sign in Eq. 2.5, whereas the lower curve originates from the minus-sign in Eq. 2.5

$$
\mathbf{q}=\left(\begin{array}{l}
q_{x} \\
q_{y}
\end{array}\right)\left(\begin{array}{c}
\hat{\mathbf{x}} \\
\hat{\mathbf{y}}
\end{array}\right)
$$

Near the Fermi level write $|\mathbf{K}| \backsim 1 / a$ with the condition $q a \ll 1$. With $q=2 \pi / \lambda$ and the previous condition one finds that $\lambda \gg a$. The origin of the name long wavelength limit approximation is found in this inequality and will be applied on the matrix element $-H_{12, \mathbf{k}}\left(\right.$ or $\left.t_{\mathbf{k}}\right)$ :

$$
t\left(e^{i \mathbf{k} \cdot \mathbf{n}_{1}}+e^{i \mathbf{k} \cdot \mathbf{n}_{2}}+e^{i \mathbf{k} \cdot \mathbf{n}_{3}}\right)
$$

where

$$
\begin{gathered}
e^{i \mathbf{k} \cdot \mathbf{n}_{1}}=e^{i \mathbf{K} \cdot \mathbf{n}_{1}} e^{i \mathbf{q} \cdot \mathbf{n}_{1}}=e^{i \frac{2 \pi}{3}} e^{i \mathbf{q} \cdot \mathbf{n}_{1}} \approx e^{i \frac{2 \pi}{3}}\left(1+i \mathbf{q} \cdot \mathbf{n}_{\mathbf{1}}\right) \\
e^{i \mathbf{k} \cdot \mathbf{n}_{2}}=e^{i \mathbf{K} \cdot \mathbf{n}_{2}} e^{i \mathbf{q} \cdot \mathbf{n}_{2}}=e^{-i \frac{2 \pi}{3}} e^{i \mathbf{q} \cdot \mathbf{n}_{2}} \approx e^{-i \frac{2 \pi}{3}}\left(1+i \mathbf{q} \cdot \mathbf{n}_{\mathbf{2}}\right) \\
e^{i \mathbf{k} \cdot \mathbf{n}_{3}}=e^{i \mathbf{K} \cdot \mathbf{n}_{3}} e^{i \mathbf{q} \cdot \mathbf{n}_{3}}=e^{-i 0} e^{i \mathbf{q} \cdot \mathbf{n}_{3}} \approx\left(1+i \mathbf{q} \cdot \mathbf{n}_{3}\right)
\end{gathered}
$$

Furthermore define $\vec{q}$ such that 


$$
\mathbf{q}=\left(\begin{array}{l}
q_{1} \\
q_{2}
\end{array}\right)
$$

Applying these approximations to the matrix elements $t_{\mathbf{k}}$ and $t_{\mathbf{k}}^{*}$ gives

$$
\begin{aligned}
& t_{\mathbf{k}}=t\left(e^{i \mathbf{k} \cdot \mathbf{n}_{1}}+e^{i \mathbf{k} \cdot \mathbf{n}_{2}}+e^{i \mathbf{k} \cdot \mathbf{n}_{3}}\right) \approx \frac{\sqrt{3}}{4} a t\left[\left(-\sqrt{3} q_{1}+q_{2}\right)-i\left(q_{1}+\sqrt{3} q_{2}\right)\right]=\frac{\sqrt{3}}{4} a t\left[q_{x}-i q_{y}\right] \\
& t_{\mathbf{k}}^{*}=t\left(e^{-i \mathbf{k} \cdot \mathbf{n}_{1}}+e^{-i \mathbf{k} \cdot \mathbf{n}_{2}}+e^{-i \mathbf{k} \cdot \mathbf{n}_{3}}\right) \approx \frac{\sqrt{3}}{4} a t\left[\left(-\sqrt{3} q_{1}+q_{2}\right)+i\left(q_{1}+\sqrt{3} q_{2}\right)\right]=\frac{\sqrt{3}}{4} a t\left[q_{x}+i q_{y}\right]
\end{aligned}
$$

In the equations above the following substitutions were performed to retrieve a simpler expression:

$$
\begin{aligned}
& q_{x}=-\sqrt{3} q_{1}+q_{2} \\
& q_{y}=q_{1}+\sqrt{3} q_{2}
\end{aligned}
$$

The matrix of Eq. 2.2 then becomes

$$
\left(\begin{array}{cc}
E_{p} & t_{\mathbf{k}} \\
t_{\mathbf{k}}^{*} & E_{p}
\end{array}\right) \approx-\frac{\sqrt{3}}{4} a t\left(\begin{array}{cc}
E_{p} & q_{x}-i q_{y} \\
q_{x}+i q_{y} & E_{p}
\end{array}\right)
$$

With $E_{p}$ set to zero and defining $-\frac{\sqrt{3}}{4} a t \equiv \hbar v_{F}$ one gets

$$
-\frac{\sqrt{3}}{4} a t\left(\begin{array}{cc}
E_{p} & q_{x}-i q_{y} \\
q_{x}+i q_{y} & E_{p}
\end{array}\right)=\hbar v_{F} q_{x}\left(\begin{array}{ll}
0 & 1 \\
1 & 0
\end{array}\right)+\hbar v_{F} q_{y}\left(\begin{array}{cc}
0 & -i \\
i & 0
\end{array}\right)
$$

The two matrices on the right-hand-side of Eq. 2.6 are defined as $\hat{\sigma}_{x}$ and $\hat{\sigma}_{y}$ respectively, which are two of the three Pauli matrices. Equation 2.6 is rewritten as

$$
\hbar v_{F} q_{x} \hat{\sigma}_{x}+\hbar v_{F} q_{y} \hat{\sigma}_{y}=\hbar v_{F} \mathbf{q} \cdot \hat{\boldsymbol{\sigma}} \equiv v_{F} \mathbf{p} \cdot \hat{\boldsymbol{\sigma}}
$$

The latter is retrieved by the substitution $\mathbf{p}=\hbar \mathbf{q}$. The Hamiltonian then reads

$$
\left(v_{F} \mathbf{p} \cdot \hat{\boldsymbol{\sigma}}\right) \psi_{p}=E_{p} \psi_{p} \text { with } \psi_{p}=\left(\begin{array}{c}
c_{1, \mathbf{p}} \\
c_{2, \mathbf{p}}
\end{array}\right) \text { and } \mathbf{p} \cdot \hat{\boldsymbol{\sigma}}=\left(\begin{array}{cc}
0 & p_{x}-i_{y} \\
p_{x}+i p_{y} & 0
\end{array}\right)
$$

The eigenvalues of the refurbished Hamiltonian are

$$
E_{\mathbf{p}}^{ \pm}= \pm v_{F} \sqrt{\left(p_{x}\right)^{2}+\left(p_{y}\right)^{2}}= \pm v_{F} p
$$

Thus a linear dispersion relation is retrieved in the vicinity of the K-point around the Fermi-level with $v_{F}$ in the order of $10^{6} \mathrm{~m} / \mathrm{s}$ [?]. Similarly, another (quasi)particle exists with a linear dispersion relation, which is the photon. Photons are massless too and exhibit a linear dispersion relation (with the speed of light). This fascinating similarity between graphene's $p_{z}$-electrons and photons inspired to consider the electrons in graphene as massless Dirac fermions. In Sec. 2.6 a detailed description of this feature is provided. 


\subsection{Density of states of graphene}

Many electronic structure related experiments and calculations are related to the density of states of the studied materials. In this subsection the density of states for graphene is derived. Figure 2.8 illustrates the electronic states in k-space for two-dimensional materials based upon the free electron model. The states that

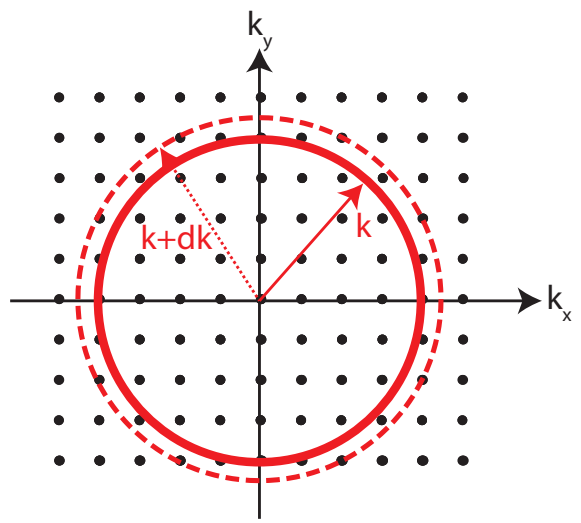

Figure 2.8 - An illustration of the states in k-space for two-dimensional materials. The density of states per unit area can be retrieved by counting the states in the area between the two circles, e.g. dashed $k+d k$ and solid $k$ lines.

have the largest contribution for conduction are located in the area between the circles of $k+d k$ and solid $k$. The size of $k$ is chosen such that it only approximates the Fermi-level, i.e. states around the K-point. The area of this shell is $\mathrm{A}_{2 D}$.

$$
A_{2 D}=\pi(k+d k)^{2}-\pi k^{2}=2 \pi k d k+\pi(d k)^{2} \approx 2 \pi k d k
$$

The term $\pi(d k)^{2}$ vanishes because $d k$ is small and $(d k)^{2}$ may be considered insignificant. The area of the circle with diameter $k$ is $\left(\frac{2 \pi}{L}\right)^{2}$, since $k_{x}$ and $k_{y}$ are $\left(\frac{2 \pi n_{x}}{L}\right)$ and $\left(\frac{2 \pi n_{y}}{L}\right)$ respectively. Here, $n$ represents the quantum number in both dimensions. The ratio between the area of the shell and the circle is proportional to the density of states per unit area as a function of energy, i.e.

$$
g(E) d E=\frac{1}{L^{2}} \frac{2 \pi k d k}{\left(\frac{2 \pi}{L}\right)^{2}}
$$

The first factor on the right-hand side is the normalization per unit area. This equation can be written as

$$
g(E)=\frac{k}{2 \pi} \frac{d k}{d E}
$$

In order to find the derivative one needs to have the dispersion relation that was previously calculated and given by Eq. 2.7. By using $\mathbf{p}=\hbar \mathbf{k}$ the dispersion 
relation transforms into

$$
E(k)=\hbar v_{F} k \quad \Longleftrightarrow \quad k=\frac{E}{\hbar v_{F}}
$$

The derivative of $\mathrm{k}$ with respect to $\mathrm{E}$ is then easily found

$$
\frac{d k}{d E}=\frac{1}{\hbar v_{F}}
$$

Substituting Eq. 2.9 and Eq. 2.10 into Eq. 2.8 gives

$$
g(E)=\frac{E}{2 \pi\left(\hbar v_{F}\right)^{2}}
$$

By taking into account spin and valley degeneracy (from $K$ and $K^{\prime}$ ) the density of states per unit area is multiplied by $2 \times 2=4$ and becomes

$$
g(E)=\frac{2 E}{\pi\left(\hbar v_{F}\right)^{2}}
$$

Thus the density of states is linearly dependent on the energy for a 2D-honeycomb lattice as graphene, which is contrary to other two-dimensional electron gas materials where the density of states is constant and independent of the energy. Finally, the density of states versus the energy is given in Fig. 2.9.

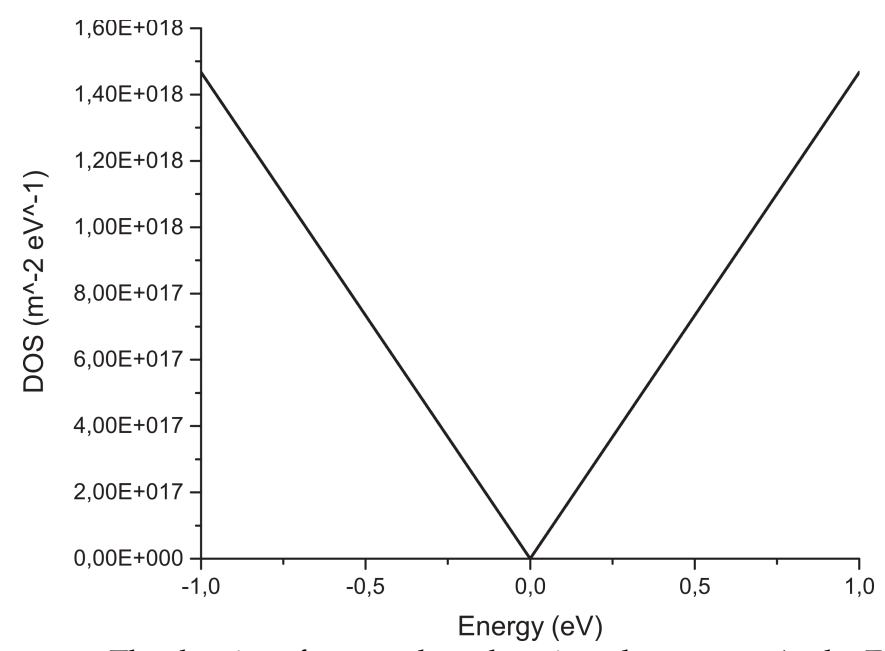

Figure 2.9 - The density of states plotted against the energy. At the Fermilevel, $\mathrm{E}=0$, no states are present. Away from the Fermi-level the curve is linear. Both properties are a direct signature of the Dirac cone. 


\subsection{Dirac equation}

The linear dispersion relation for electrons in graphene shows similarity with the dispersion relation for photons. Hence, it is said that the $p_{z}$-electrons in graphene are relativistic massless Dirac fermions. A serious consequence is that a quantum mechanical view solely would not suffice to understand the physics that the electrons undergo. Therefore the special theory of relativity must be taken into account. An attempt to tackle this problem is to make use of the Klein-Gordon equation, but this theory is only valid for particles with zero spin. Since a fermion like an electron does not exhibit zero spin, the Klein-Gordon approach is useless in this case. The way to go is by following the Dirac equation [?].

Firstly, the time-dependent Schrödinger equation is needed to describe the quantum mechanics:

$$
\left[\imath \hbar \frac{\partial}{\partial t}-\hat{H}\right] \psi(x, t)=0
$$

Here $\hbar$ denotes the Planck-constant, $\hat{H}$ the Hamiltonian operator and $\psi(x, t)$ the one dimensional time dependent wave function. The special theory of relativity is given by the following dispersion relation:

$$
E^{2}=p^{2} c^{2}+m^{2} c^{4}
$$

or

$$
E^{2}-p^{2} c^{2}-m^{2} c^{4}=0
$$

Here $E$ is the energy, whereas $p$ denotes the momentum, $m$ the mass and $c$ the speed of light. The next step is to factorize Eq. 2.13 into

$$
E^{2}-p^{2} c^{2}-m^{2} c^{4}=\left(E+\alpha p c+\beta m c^{2}\right)\left(E-\alpha p c-\beta m c^{2}\right)=0
$$

Clearly $E, \alpha p c$ and $\beta m c^{2}$ are positive and therefore the first factor after factorization cannot result in a zero outcome. Therefore, the second factor must be set zero.

$$
E-\alpha p c-\beta m c^{2}=0 \Longleftrightarrow E=\alpha p c+\beta m c^{2}
$$

Dirac's approach is to transforms the energy and momentum into operators.

$$
\begin{gathered}
E=\alpha p c+\beta m c^{2} \Longleftrightarrow \hat{H}=\alpha \hat{p} c+\beta m c^{2} \\
\text { with } \hat{p}=-i \hbar \frac{\partial}{\partial x}
\end{gathered}
$$


Upon substituting the new Hamiltonian operator into the time-dependent Schrödinger equation (e.g. Eq. 2.12) the first glimpse of the Dirac-equation is retrieved:

$$
\left[\imath \hbar \frac{\partial}{\partial t}-\left(\alpha c\left(-\imath \hbar \frac{\partial}{\partial x}\right)+\beta m c^{2}\right)\right] \psi(x, t)=0
$$

The parameters $\alpha$ and $\beta$ can be found by expanding Eq. 2.14

$$
\left(E+\alpha p c+\beta m c^{2}\right)\left(E-\alpha p c-\beta m c^{2}\right)=E^{2}-\alpha^{2} p^{2} c^{2}-\beta^{2} m^{2} c^{4}-(\alpha \beta+\beta \alpha) p m c^{3}
$$

and then by making the latter equal with left-hand side of Eq. 2.14. The following set of equations needs to be solved

$$
\begin{gathered}
\alpha^{2}=1 \\
\beta^{2}=1 \\
\alpha \beta+\beta \alpha=0
\end{gathered}
$$

To satisfy the equalities as given above $\alpha$ and $\beta$ need to be $2 \times 2$-matrices. The solutions correspond with two Pauli matrices.

$$
\alpha=\left(\begin{array}{ll}
0 & 1 \\
1 & 0
\end{array}\right)=\sigma_{x} \text { and } \beta=\left(\begin{array}{rr}
1 & 0 \\
0 & -1
\end{array}\right)=\sigma_{z}
$$

Next a spinor $\Psi(x, t)$ is introduced as

$$
\Psi(x, t)=\left(\begin{array}{l}
\psi_{1}(x, t) \\
\psi_{2}(x, t)
\end{array}\right)
$$

The Fermi-Dirac equation follows from the substitution of the Pauli matrices and the spinor into Eq. 2.15:

$$
\left[\imath \hbar \frac{\partial}{\partial t}-\left(c \sigma_{x}\left(-\imath \hbar \frac{\partial}{\partial x}\right)+m c^{2} \sigma_{z}\right)\right]\left(\begin{array}{c}
\psi_{1}(x, t) \\
\psi_{2}(x, t)
\end{array}\right)=0
$$

Equation 2.16 is only valid for one-dimensional systems, but graphene is twodimensional. The extension to two-dimensions is done fairly easily.

$$
\left[\imath \hbar \frac{\partial}{\partial t}+\imath \hbar c\left(\sigma_{x} \frac{\partial}{\partial x}+\sigma_{y} \frac{\partial}{\partial y}\right)-m c^{2} \sigma_{z}\right]\left(\begin{array}{l}
\psi_{1}(x, y, t) \\
\psi_{2}(x, y, t)
\end{array}\right)=0
$$

The third Pauli-matrix introduced here is defined as

$$
\sigma_{y}=\left(\begin{array}{cc}
0 & -i \\
i & 0
\end{array}\right)
$$

The solution to the Fermi-Dirac equation is given by

$$
\Psi(x, y, t)=e^{-\frac{l}{\hbar} E t} \boldsymbol{\Phi}(x, y)
$$


The stationary solution of the time-independent Schrödinger equation is described by $\boldsymbol{\Phi}(x, y)$. Now by using the time-dependent wave function as given above in Eq. 2.17 it becomes possible to derive the effective mass and Fermi velocity of graphene. For the sake of convenience the $\psi_{1}(x, y, t)$ and $\psi_{2}(x, y, t)$ are written as $\psi_{1}$ and $\psi_{2}$ respectively.

$$
\imath \hbar \frac{\partial}{\partial t} \boldsymbol{\Psi}(x, y, t)=m c^{2}\left(\begin{array}{c}
\psi_{1} \\
-\psi_{2}
\end{array}\right)-i \hbar c \sigma_{x} \frac{\partial}{\partial x}\left(\begin{array}{l}
\psi_{1} \\
\psi_{2}
\end{array}\right)-i \hbar c \sigma_{y} \frac{\partial}{\partial y}\left(\begin{array}{l}
\psi_{1} \\
\psi_{2}
\end{array}\right)
$$

It is evident that the left-hand side of Eq. A.1 will give us the eigenvalues, or in

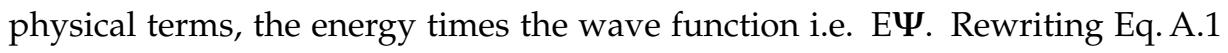
by filling in the Pauli matrices one gets

$$
E\left(\begin{array}{c}
\psi_{1} \\
\psi_{2}
\end{array}\right)=m c^{2} \sigma_{z}\left(\begin{array}{c}
\psi_{1} \\
\psi_{2}
\end{array}\right)+\hbar c\left(\begin{array}{c}
-\psi_{2 y}-i \psi_{2 x} \\
\psi_{1 y}-i \psi_{1 x}
\end{array}\right)
$$

Here the subscripts $x$ and $y$ are representing the derivative with respect to $x$ and y respectively. It is previously stated in Eq. 2.7 that the energy must be linearly dependent on the momentum, where the momentum is $\mathbf{p}=\hbar \mathbf{k}$. If Eq. A.2 is analyzed carefully and matched with the linear dispersion relation, then two conditions must be satisfied in order to validate the linear disperion relation, namely:

$$
m=0
$$

and

$$
\left(\begin{array}{c}
-\psi_{2 y}-i \psi_{2 x} \\
\psi_{1 y}-i \psi_{1 x}
\end{array}\right)=k\left(\begin{array}{c}
\psi_{1} \\
\psi_{2}
\end{array}\right)
$$

The wave functions can be solved as will be shown in the subsection below. If Eq. 2.20 and Eq. 2.21 are filled into Eq. A.2 one retrieves

$$
E\left(\begin{array}{l}
\psi_{1} \\
\psi_{2}
\end{array}\right)=\hbar c k\left(\begin{array}{l}
\psi_{1} \\
\psi_{2}
\end{array}\right)
$$

The wave functions cancel out and all that is left is

$$
E=\hbar c|\mathbf{k}|=c \hbar|\mathbf{k}|=c|\mathbf{p}| \Longleftrightarrow E=v_{F}|\mathbf{p}|
$$

This also shows that in this relativistic approach the Fermi-velocity is replaced by the speed of light. Combined with Eq. 2.20 this outcome shows that the electrons of graphene (silicene and germanene) exhibit massless Dirac fermion behavior:

$$
\begin{aligned}
& v_{F}=c \\
& m=0
\end{aligned}
$$




\section{Solving the Fermi-Dirac equation}

Equation 2.21 is comprised of two differential equations given by each row. The first row defines $\psi_{1}$ as a sum of derivatives of $\psi_{2}$ and can be substituted in the left-hand side of the second row. This leads to a partial differential equation with only one parameter, i.e. $\psi_{2}$. By finding $\psi_{2}$ it is possible to compute $\psi_{1}$. The partial differential equation reads:

$$
\psi_{2 y y}+\psi_{2 x x}=-k^{2} \psi_{2}
$$

A possible solution (trial solution) to Eq. 2.24 is

$$
\psi_{2}=A e^{\frac{i}{2} \sqrt{2} k(x+y)}+B e^{-\frac{i}{2} \sqrt{2} k(x+y)}
$$

The parameters A and B are constants and normalize the wave function. Now that $\psi_{2}$ is known, $\psi_{1}$ can be derived from the first row equation of Eq. 2.21:

$$
\psi_{1}=\left(\frac{1}{2} \sqrt{2}-\frac{i}{2} \sqrt{2}\right) A e^{\frac{i}{2} \sqrt{2} k(x+y)}-\left(\frac{1}{2} \sqrt{2}-\frac{i}{2} \sqrt{2}\right) B e^{-\frac{i}{2} \sqrt{2} k(x+y)}
$$

Or more conveniently

$$
\psi_{1}=C e^{\frac{i}{2} \sqrt{2} k(x+y)}-D e^{-\frac{i}{2} \sqrt{2} k(x+y)}
$$

with $\mathrm{C}$ and D normalizing the wave function.

So far in this section no potential was considered. A potential function is easily included in the Fermi-Dirac equation (see Eq. 2.17) resulting in

$$
\left[\imath \hbar \frac{\partial}{\partial t}+\imath \hbar c\left(\sigma_{x} \frac{\partial}{\partial x}+\sigma_{y} \frac{\partial}{\partial y}\right)-m c^{2} \sigma_{z}+e \phi(x, y, t)\right]\left(\begin{array}{l}
\psi_{1}(x, y, t) \\
\psi_{2}(x, y, t)
\end{array}\right)=0
$$

with $\phi(x, y, t)$ the potential function in two dimensions. Solving such an equation can be quite cumbersome and is not addressed in this thesis. 


\subsection{Tight-Binding Graphene-edges}

Graphene edges are theoretically terminated in two manners, namely zigzag and armchair [?,?] (excluding defects like Stone-Wales defects [?,?]). The properties that these edges exhibit differ substantially with respect to each other. In this Section the tight-binding method for both edges is calculated. The calculations are also extended to nanoribbons with different widths.

\subsubsection{Armchair Edges}

A schematic of the armchair edge is given in Fig. 2.10. Here, only the first three dimer-rows are indicated. Firstly, the tight-binding method is solved for the single-strip $(\mathrm{N}=1)$ situation. Next, the same is done for the double strip configuration. From here on, the total number of strips is extended and a general solution is given for any width.

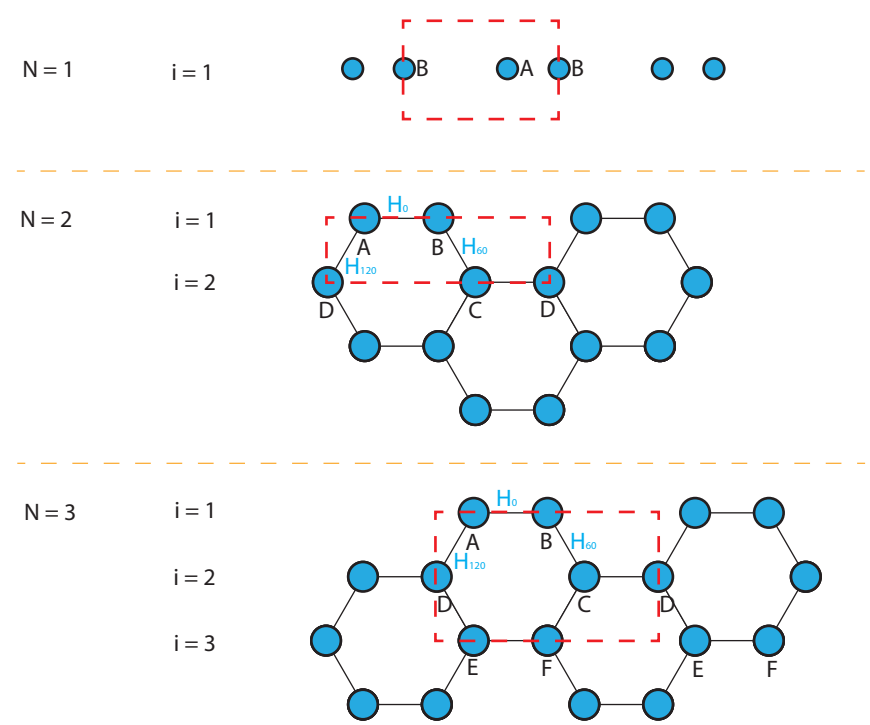

Figure 2.10 - The armchair edges are characterized by dimer rows that are shifted horizontally in each alternating row. The single strip configuration is given in the top panel. The unit cell consists of two atoms. In this example atom $\mathrm{A}$ is coupled to its nearest and next-nearest neighbour atom B. In the middle panel three rows are added to make the armchair more clear. The first two rows form the double strip problem. Three types of neighbours exist, namely those who are aligned parallel to the horizontal component $\left(H_{0}\right)$ and those who point to either lower left $\left(H_{120}\right)$ or lower right $\left(H_{60}\right)$. Four atoms exist in the unit cell for the double strip problem. In the lower panel the triple strip problem is shown. In this unit cell six atoms reside. 


\section{Single strip, $\mathbf{N}=1$}

The top panel of Fig. 2.10 shows a single strip of the armchair edge. Please note that the unit cell is one-dimensional and that the vertical components of the red-dashed rectangle is only used to indicate the unit cell. The unit cell comprises two atoms, namely $\mathrm{A}$ and $\mathrm{B}$. In this exemplary unit cell atom $\mathrm{A}$ is connected to two atoms $\mathrm{B}$ with separated by $d$ and $2 d$ respectively. Here, the length $d$ corresponds with the atom-to-atom distance $\frac{a}{\sqrt{3}}=1.42 \AA$ where $a=2.46 \AA$. The first strip is a one-dimensional chain of atoms with a unit cell width of $a \sqrt{3}$. The Hamiltonian matrix will therefore become a $(2 \times 2)$-matrix. Coupling between atom $\mathrm{A}$ and the next-nearest neighbour atom $\mathrm{B}$ must be taken into account in order to prevent an entirely flat band. Therefore, the first strip violates the nearest-neighbour approximation and becomes a next-nearestneighbour approximation. The matrix elements are given by:

$$
\begin{gathered}
H_{A A}=\left\langle\chi_{A}(\mathbf{r})|\hat{H}| \chi_{A}(\mathbf{r})\right\rangle=\epsilon_{p} \\
H_{A B}=\sum_{j=1}^{2} e^{i k \cdot n_{j}}\left\langle\chi_{A}(\mathbf{r})|\hat{H}| \chi_{B}\left(\mathbf{r}-\mathbf{n}_{\mathbf{j}}\right)\right\rangle=-t_{1} e^{i k d}-t_{2} e^{-2 i k d}=t_{k} \\
H_{B A}=\sum_{j=1}^{3} e^{i k \cdot n_{j}}\left\langle\chi_{B}(\mathbf{r})|\hat{H}| \chi_{A}\left(\mathbf{r}-\mathbf{n}_{\mathbf{j}}\right)\right\rangle=-t_{1} e^{-i k d}-t_{2} e^{2 i k d}=t_{k}^{*} \\
H_{B B}=\left\langle\chi_{B}(\mathbf{r})|\hat{H}| \chi_{B}(\mathbf{r})\right\rangle=\epsilon_{p}
\end{gathered}
$$

Note that both exponents in $H_{A B}$ and $H_{B A}$ have different directions and magnitudes. The overlap integral between nearest neighbours and next-nearest neighbours is different. The nearest neighbour overlap integral must be larger than the next-nearest neighbour overlap integral. This gives rise to two distinct hopping parameters $t_{1}$ and $t_{2}$. The former hopping parameter is equal to the hopping parameter that has been used for all previous carbon structures, i.e. $3 \mathrm{eV}$. It is required that $t_{2}$ should be smaller than $t_{1}$.

$$
\left(\begin{array}{cc}
E_{k}-\epsilon_{p} & -t_{k} \\
-t_{k}^{*} & E_{k}-\epsilon_{p}
\end{array}\right)\left(\begin{array}{l}
c_{1} \\
c_{2}
\end{array}\right)=0\left(\begin{array}{l}
c_{1} \\
c_{2}
\end{array}\right)
$$

The matrix is solved by computing the determinant and writing $E_{k}$ as a function of $k$. The analytical solution is not given here. The result is shown in Fig. 2.11(a). Not surprisingly, a harmonic function is retrieved for the one-dimensional chain of atoms. The hopping parameters $t_{1}$ and $t_{2}$ were chosen to be $-3.0 \mathrm{eV}$ and $0.75 \mathrm{eV}$ respectively. The single strip configuration has a band gap of $2.25 \mathrm{eV}$ and is therefore a semiconductor. 


\section{Double strips, $\mathrm{N}=2$}

Now a two-dimensional unit cell is defined with a vertical component as long as the distance between the first and second strip. In this unit cell four atoms are located and are called $\mathrm{A}, \mathrm{B}, \mathrm{C}$ and $\mathrm{D}$ respectively. The Hamiltonian-matrix becomes a $(4 \times 4)$-matrix.

$$
\left(\begin{array}{llll}
H_{A A} & H_{A B} & H_{A C} & H_{A D} \\
H_{B A} & H_{B B} & H_{B C} & H_{B D} \\
H_{C A} & H_{C B} & H_{C C} & H_{C D} \\
H_{D A} & H_{D B} & H_{D C} & H_{D D}
\end{array}\right)
$$

All matrix elements consisting of the same letters are defined as $\epsilon_{p}$. Within the nearest-neighbor approximation atoms $\mathrm{B}$ and $\mathrm{D}$ and atoms $\mathrm{A}$ and $\mathrm{C}$ do not overlap. Therefore, the matrix elements $H_{A C}$ and $H_{B D}$ are zero. Similarly, their complex conjugate, $H_{C A}$ and $H_{D B}$, are also zero. The matrix is now written as:

$$
\left(\begin{array}{cccc}
\epsilon_{p} & H_{A B} & 0 & H_{A D} \\
H_{B A} & \epsilon_{p} & H_{B C} & 0 \\
0 & H_{C B} & \epsilon_{p} & H_{C D} \\
H_{D A} & 0 & H_{D C} & \epsilon_{p}
\end{array}\right)
$$

For the remaining unknown matrix elements one can impose the complex conjugate rule $H_{i j}=H_{j i}^{*}$ to make the calculation easier. There are only three vectors that describe the neighbouring bonds. There is a horizontal line like from atom A to atom $B$ or from atom $C$ to atom $D$ as shown in Fig. 2.10. The matrix element for such neighbours is called $H_{0}$ as it has $0^{\circ}$ rotation with respect to the positive $\mathrm{x}$-direction. Then there are neighbours that are positioned from each other with a rotation of $240^{\circ}$, for example from atom A to atom D. A rotation of $240^{\circ}$ is equivalent to a rotation of $-120^{\circ}$. For sake of convenience this matrix element will be called $H_{120}$. The last neighbouring position has a rotation of $300^{\circ}$, or $-60^{\circ}$ and is called $H_{60}$, e.g. from atom $\mathrm{B}$ to atom $\mathrm{C}$. Below the matrix elements are given in full extend.

$$
\left\{\begin{array}{l}
H_{0}=e^{i k_{x} d}\left\langle\chi_{A}(\mathbf{r})|\hat{H}| \chi_{B}(\mathbf{r}-\mathbf{d})\right\rangle=-t e^{i k_{x} d} \\
H_{60}=e^{i k_{x} \frac{d}{2}-i k_{y} \frac{d \sqrt{3}}{2}}\left\langle\chi_{B}(\mathbf{r})|\hat{H}| \chi_{C}(\mathbf{r}-\mathbf{d})\right\rangle=-t e^{i k_{x} \frac{d}{2}-i k_{y} \frac{d \sqrt{3}}{2}} \\
H_{120}=e^{-i k_{x} \frac{d}{2}-i k_{y} \frac{d \sqrt{3}}{2}}\left\langle\chi_{A}(\mathbf{r})|\hat{H}| \chi_{D}(\mathbf{r}-\mathbf{d})\right\rangle=-t e^{-i k_{x} \frac{d}{2}-i k_{y} \frac{d \sqrt{3}}{2}}
\end{array}\right.
$$

With these freshly defined matrix elements one can construct the Hamiltonianmatrix for the two strips configuration of the armchair edge.

$$
\left(\begin{array}{cccc}
\epsilon_{p} & H_{0} & 0 & H_{120} \\
H_{0}^{*} & \epsilon_{p} & H_{60} & 0 \\
0 & H_{60}^{*} & \epsilon_{p} & H_{0} \\
H_{120}^{*} & 0 & H_{0}^{*} & \epsilon_{p}
\end{array}\right)
$$


The eigenvalues of the matrix give the solution to the Schrödinger equation and therefore the band structure of the armchair edge. The result is shown in Fig. 2.11(b). The double stripped armchair edge is metallic at the $\Gamma$-point. Its nature differs substantially from the single strip configuration where the nanoribbon is semiconducting.

\section{First three strips}

Analogously to the two-strips configuration the matrix elements $H_{0}, H_{60}$ and $H_{120}$ are used to develop the Hamiltonian matrix for the three strips configuration. Now there are six atoms in the unit cell and thus a $6 \times 6$-matrix is constructed. The first $4 \times 4$-compartment of the matrix is equal to that of the two-strips system. The matrix that needs to be solved is:

$$
\left(\begin{array}{cccccc}
\epsilon_{p} & H_{0} & 0 & H_{120} & H_{A E} & H_{A F} \\
H_{0}^{*} & \epsilon_{p} & H_{60} & 0 & H_{B E} & H_{B F} \\
0 & H_{60}^{*} & \epsilon_{p} & H_{0} & H_{C E} & H_{C F} \\
H_{120}^{*} & 0 & H_{0}^{*} & \epsilon_{p} & H_{D E} & H_{D F} \\
H_{E A} & H_{E B} & H_{E C} & H_{E D} & H_{E E} & H_{E F} \\
H_{F A} & H_{F B} & H_{F C} & H_{F D} & H_{F E} & H_{F F}
\end{array}\right)
$$

The matrix element $H_{C F}$ corresponds to $H_{120}$ as one can see from Fig. 2.10. Similarly, the new matrix elements $H_{D E}=H_{60}$ and $H_{E F}=H_{0}$ are found. By using $H_{i i}=\epsilon_{p}$ and that all next-nearest neighbours (and beyond) are zero this matrix simplifies to:

$$
\left(\begin{array}{cccccc}
\epsilon_{p} & H_{0} & 0 & H_{120} & 0 & 0 \\
H_{0}^{*} & \epsilon_{p} & H_{60} & 0 & 0 & 0 \\
0 & H_{60}^{*} & \epsilon_{p} & H_{0} & 0 & H_{120} \\
H_{120}^{*} & 0 & H_{0}^{*} & \epsilon_{p} & H_{60} & 0 \\
0 & 0 & 0 & H_{60}^{*} & \epsilon_{p} & H_{0} \\
0 & 0 & H_{120}^{*} & 0 & H_{0}^{*} & \epsilon_{p}
\end{array}\right)
$$

The eigenvalues of this matrix provide the band diagram of the three-stripped armchair edge as shown in Fig,2.11(c). In this configuration the armchair nanoribbon is clearly semiconducting. So far, the armchair nanoribbon has showed various characteristics. For $\mathrm{N}=1$ and $\mathrm{N}=3$ it is clearly semiconducting. In case of $\mathrm{N}=3$ there is a flat band present, which was not the case for $\mathrm{N}=1$. The second configuration, $\mathrm{N}=2$, is metallic. The width seems to influence the electronic properties enormously and will be further studied in a generalized way. 


\section{Armchair: a general solution for the $\mathbf{N}^{\text {th }}$-stripped nanoribbon}

Basically the known $(\mathrm{N}-1)^{t h}$-strip can be extended to the $\mathrm{N}^{\text {th }}$ strip by adding two atoms in the expanded unit cell. This means that the matrix gets two new rows and two new columns. For every expansion in the nearest neighbour approximation it holds that the new matrix elements are as follows:

$$
\begin{gathered}
\left\{\begin{array}{l}
H_{2 N-3,2 N}=H_{120} \\
H_{2 N, 2 N-3}=H_{120}^{*}
\end{array}\right. \\
\left\{\begin{array}{l}
H_{2 N-2,2 N-1}=H_{60} \\
H_{2 N-1,2 N-2}=H_{60}^{*}
\end{array}\right. \\
\left\{\begin{array}{l}
H_{2 N-1,2 N}=H_{0} \\
H_{2 N, 2 N-1}=H_{0}^{*}
\end{array}\right. \\
\left\{\begin{array}{l}
H_{2 N-1,2 N-1}=\epsilon_{p} \\
H_{2 N, 2 N}=\epsilon_{p}
\end{array}\right.
\end{gathered}
$$

Eventually the following matrix is acquired.

$$
\left(\begin{array}{cccccc}
\ddots & \ddots & \ddots & \ddots & 0 & 0 \\
\ddots & \ddots & \ddots & \ddots & 0 & 0 \\
\ddots & \ddots & \ddots & \ddots & 0 & H_{120} \\
\ddots & \ddots & \ddots & \ddots & H_{60} & 0 \\
0 & 0 & 0 & H_{60}^{*} & \epsilon_{p} & H_{0} \\
0 & 0 & H_{120}^{*} & 0 & H_{0}^{*} & \epsilon_{p}
\end{array}\right)
$$

Computing the N-strip armchair edge is performed by first constructing the two-strip matrix. From here, at each loop one strip is added to the matrix, i.e. the matrix is expanded with two rows and two columns, until the $\mathrm{N}^{\text {th }}$ strip is reached. The evaluation of the eigenvalues leads to the band diagram. Figure 2.11 shows the band diagrams from some widths. For $\mathrm{N}=5$ the armchair nanoribbon is again metallic, whilst for $\mathrm{N}=4$ and $\mathrm{N}=6$ the nanoribbon is semiconducting.

To illustrate the dependence of the electronic properties on the width of the nanoribbon the band gap was calculated for each width. The band gap is plotted against the width of the nanoribbon and is given in Fig. 2.12. The result is in strong agreement with other calculation studies [?, ?, ?] and the width dependence of the band gap (in case of semiconducting nanoribbons) is dominated by quantum confinement. Whenever the width of the nanoribbon corresponds with $3 n+2$ with $n$ a positive integer the nanoribbon is metallic. In all other 

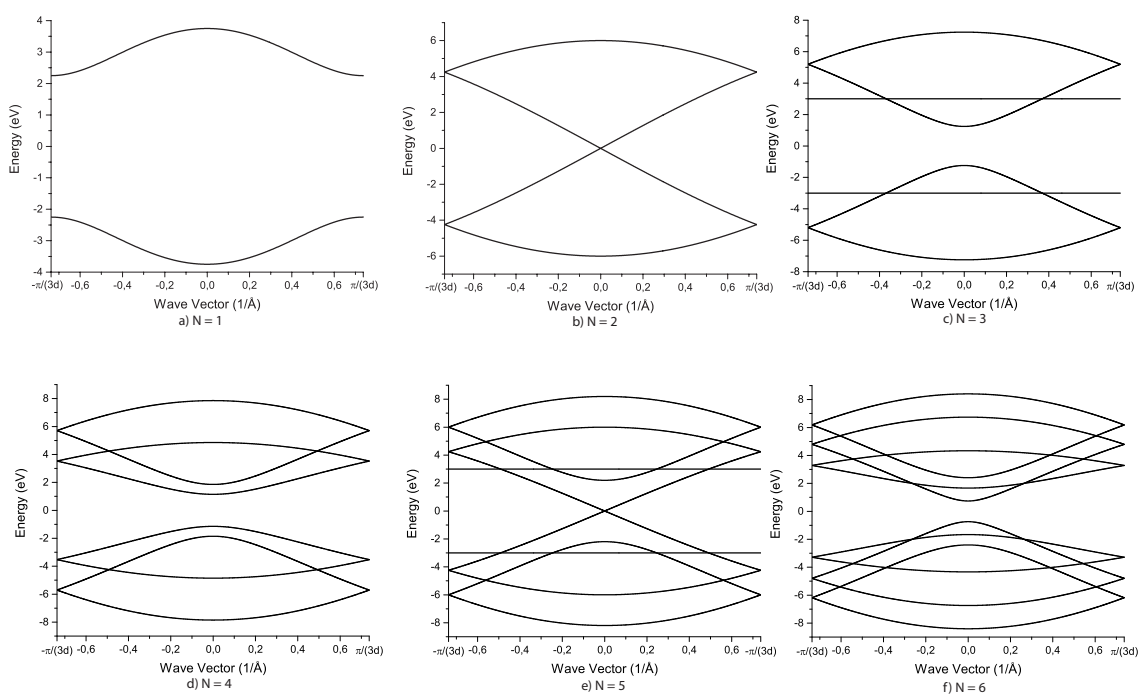

Figure 2.11 - The band structures of the armchair graphene nanoribbon with various width, i.e. 1 (a), 2 (b), 3 (c), 4 (d), 5 (e) and 6 (f). Depending on the width of the nanoribbon the armchair nanoribbon is either metallic or semiconducting (with different band gap for each width that is semiconducting).

cases the armchair nanoribbon is semiconducting. These results are only true when the tight-binding method is applied. On the contrary, first-principle calculations show that the $3 n+2$ nanoribbons, i.e. the metallic nanoribbon from tight-binding, have a non-zero band gap [?]. Also, the tight-binding method offers a pattern in the size of the band gap of the semiconducting nanoribbons $\left(\Delta_{3 n}>\Delta_{3 n+1}\right)$. Again, first-principle calculations provide an opposite conclusion of which the difference is explained by inducing hydrogen passivation at the edges and decrements in the interatomic distances at the edges [?]. A Scanning Tunneling Microscopy study gives evidence that the results from first-principle calculations fit better than the tight-binding method [?]. 


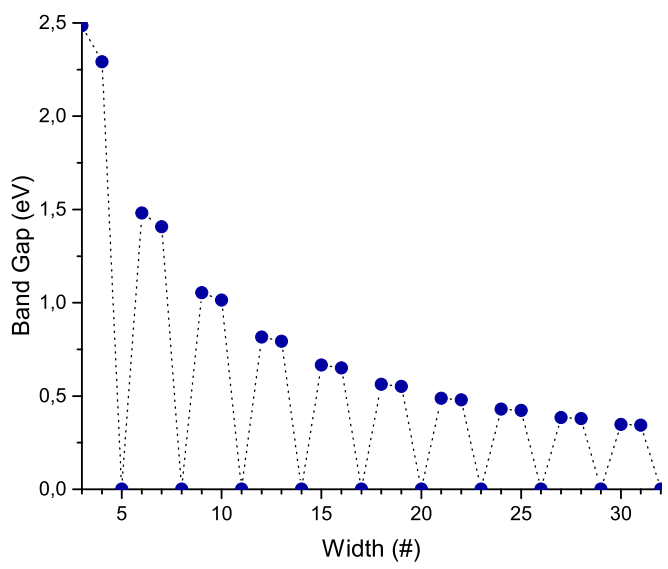

Figure 2.12 - The band gap for each width was calculated and plotted versus the width. The metallic states occur every three strips starting off at two strips. One strip less than the metallic strips typically have lower band gaps than two strips before. The difference in band gap between each consecutive semiconducting strip seems to get smaller as the nanoribbons widen.

\subsubsection{Zigzag Edges}

The zigzag edges are calculated in the same fashion as what was done for the armchair edges. The width-dependence of the electronic structure has been investigated for the zigzag edges. Analogous to the armchair edges, this subsection involves the expansion of the Hamiltonian matrix towards solutions of N-strips configurations.

\section{Two strips, $\mathbf{N}=2$}

The two strips problem can be approached in two ways. One way to solve it is to combine two individual strips rather than to make use of a unit cell that covers atoms from both the first strip and the second strip. Firstly, the combination of two individual strips is calculated

The total Hamiltonian for the i'th strip (as in Fig.2.13) is given by the sum of three Hamiltonians:

$$
\hat{H}_{t o t}=\hat{H}_{n, i-1}+\hat{H}_{m, i}+\hat{H}_{n, i+1}
$$

The Hamiltonians $\hat{H}_{n, i-1}$ and $\hat{H}_{n, i+1}$ are (2x2)-matrices that couple the neighboring strips to the $i^{\prime}$ th strip. The Hamiltonian $\hat{H}_{m, i}$ is also a $2 \times 2$-matrix, but one that 
(a) (b) $\quad$ (c)

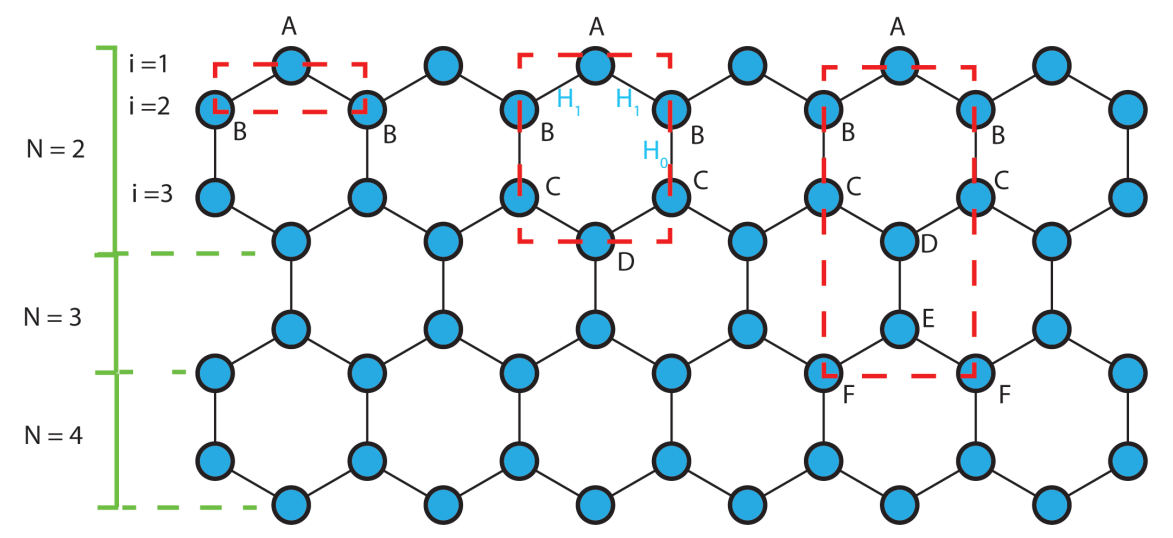

Figure 2.13 - The zigzag edges are characterized by dimer rows. The single strip configuration is given in the top panel. The unit cell consists of two atoms. In this example atom $\mathrm{A}$ is coupled to its nearest neighbour atom $\mathrm{B}$. For part (b) the unit cell for $\mathrm{N}=2$ is depicted. Two forms of neighbouring is present here. One that is connected to only one other atom in a vertical direction, $\mathrm{B}$ to $\mathrm{C}$. This is called $H_{0}$. The other one is a connection of an atom to two other atoms from another row, e.g. $\mathrm{C}$ to $\mathrm{D}$ or $\mathrm{A}$ to $\mathrm{B}$. The corresponding matrix element is $H_{1}$. Part (c) illustrates the $\mathrm{N}=3$ problem comprising six atoms.

involves a one-dimensional array of carbon atoms located $a=2.46 \AA$ away from each other. The labels $\mathrm{m}$ and $\mathrm{n}$ represent the main strip and the neighboring strips respectively. The coupling Hamiltonians are given by:

$$
\hat{H}_{n, i-1}=\left(\begin{array}{cc}
0 & 2 t \\
0 & 0
\end{array}\right) \quad \text { and } \quad \hat{H}_{n, i+1}=\hat{H}_{n, i-1}^{\dagger}=\left(\begin{array}{cc}
0 & 0 \\
-2 t & 0
\end{array}\right)
$$

The main Hamiltonian is less straightforward, since it is a $(4 \times 4)$-matrix with four coefficients originating from the two strips (that are contributing two coefficients each). Firstly, the Hamiltonian matrix for the one-dimensional carbon chain needs to be acquired. Its matrix elements are

$$
\begin{gathered}
H_{A_{1} A_{1}}=\left\langle\chi_{A_{1}}(\mathbf{r})|\hat{H}| \chi_{A_{1}}(\mathbf{r})\right\rangle=\epsilon_{p} \\
H_{A_{1} A_{2}}=\sum_{j=1}^{2} e^{i k \cdot n_{j}}\left\langle\chi_{A_{1}}(\mathbf{r})|\hat{H}| \chi_{A_{2}}\left(\mathbf{r}-\mathbf{n}_{\mathbf{j}}\right)\right\rangle=-t\left(e^{-i k a}+e^{i k a}\right)=t_{k} \\
H_{A_{2} A_{1}}=\sum_{j=1}^{2} e^{i k \cdot n_{j}}\left\langle\chi_{A_{2}}(\mathbf{r})|\hat{H}| \chi_{A_{1}}\left(\mathbf{r}-\mathbf{n}_{\mathbf{j}}\right)\right\rangle=-t\left(e^{i k a}+e^{-i k a}\right)=t_{k}^{*} \\
H_{A_{2} A_{2}}=\left\langle\chi_{A_{2}}(\mathbf{r})|\hat{H}| \chi_{A_{2}}(\mathbf{r})\right\rangle=\epsilon_{p}
\end{gathered}
$$


Here only the first strip is considered and the indices 1 and 2 indicate an atom and its neighbour. The equation that needs to be solved for the $i^{\prime}$ th strip is then written as:

$$
\hat{H}_{n, i-1} \vec{c}_{i-1}+\hat{H}_{m, i} \vec{c}_{i}+\hat{H}_{n, i+1} \vec{c}_{i+1}=E_{k} \vec{c}_{i}
$$

For the zigzag-edge two equations need to be solved, i.e. one for the terminating edge (first strip) and the non-terminating edge (second strip).

$$
\begin{aligned}
& \hat{H}_{m, 1} \vec{c}_{1}+\hat{H}_{n, 2} \vec{c}_{2}=E_{k} \vec{c}_{1} \Longleftrightarrow\left[E_{k} I_{2}-\hat{H}_{m, 1}\right] \vec{c}_{1}-\hat{H}_{n, 2} \vec{c}_{2}=0 \\
& \hat{H}_{n, 1} \vec{c}_{1}+\hat{H}_{m, 2} \vec{c}_{2}=E_{k} \vec{c}_{2} \Longleftrightarrow\left[E_{k} I_{2}-\hat{H}_{m, 2}\right] \vec{c}_{2}-\hat{H}_{n, 1} \vec{c}_{1}=0
\end{aligned}
$$

Note that any contributions coming from the third strip, i.e. $\vec{c}_{3}=0$, has been neglected in Eq. 2.36b. The two equations can be rewritten as:

$$
\begin{aligned}
\left(\begin{array}{cc}
E_{k}-\epsilon_{p} & -t_{k} \\
-t_{k}^{*} & E_{k}-\epsilon_{p}
\end{array}\right)\left(\begin{array}{l}
c_{1 x} \\
c_{1 y}
\end{array}\right)-\left(\begin{array}{cc}
0 & 0 \\
-2 t & 0
\end{array}\right)\left(\begin{array}{l}
c_{2 x} \\
c_{2 y}
\end{array}\right) & =0 \\
\left(\begin{array}{cc}
E_{k}-\epsilon_{p} & -t_{k} \\
-t_{k}^{*} & E_{k}-\epsilon_{p}
\end{array}\right)\left(\begin{array}{l}
c_{2 x} \\
c_{2 y}
\end{array}\right)-\left(\begin{array}{cc}
0 & 2 t \\
0 & 0
\end{array}\right)\left(\begin{array}{l}
c_{1 x} \\
c_{1 y}
\end{array}\right) & =0
\end{aligned}
$$

This leads to four equations that must be solved and are rewritten as follows:

$$
\left(\begin{array}{cccc}
E_{k}-\epsilon_{p} & -t_{k} & 0 & 0 \\
-t_{k}^{*} & E_{k}-\epsilon_{p} & 2 t & 0 \\
0 & -2 t & E_{k}-\epsilon_{p} & -t_{k} \\
0 & 0 & -t_{k}^{*} & E_{k}-\epsilon_{p}
\end{array}\right)\left(\begin{array}{l}
c_{1 x} \\
c_{1 y} \\
c_{2 x} \\
c_{2 y}
\end{array}\right)=0\left(\begin{array}{c}
c_{1 x} \\
c_{1 y} \\
c_{2 x} \\
c_{2 y}
\end{array}\right) \Longrightarrow\left|\begin{array}{cccc}
E_{k}-\epsilon_{p} & -t_{k} & 0 & 0 \\
-t_{k}^{*} & E_{k}-\epsilon_{p} & 2 t & 0 \\
0 & -2 t & E_{k}-\epsilon_{p} & -t_{k} \\
0 & 0 & -t_{k}^{*} & E_{k}-\epsilon_{p}
\end{array}\right|=0
$$

The result is given in Fig 2.14(a). Before the results are discussed the second approach is calculated below. The unit cell now comprises of two atoms, namely atom $\mathrm{A}$ and atom $\mathrm{B}$ as given in Fig. 2.13. The matrix elements are given by:

$$
\begin{gathered}
H_{A A}=\left\langle\chi_{A}(\mathbf{r})|\hat{H}| \chi_{A}(\mathbf{r})\right\rangle=\epsilon_{p} \\
H_{A B}=\sum_{j=1}^{2} e^{i k \cdot n_{j}}\left\langle\chi_{A}(\mathbf{r})|\hat{H}| \chi_{B}\left(\mathbf{r}-\mathbf{n}_{\mathbf{j}}\right)\right\rangle=-t\left(e^{i k_{x} \frac{a}{2}-i k_{y} \frac{1}{2} \sqrt{3} a}+e^{-i k_{x} \frac{a}{2}-i k_{y} \frac{1}{2} \sqrt{3} a}\right)=t_{k} \\
H_{B A}=H_{A B}^{*}=t_{k}^{*} \\
H_{B B}=\left\langle\chi_{B}(\mathbf{r})|\hat{H}| \chi_{B}(\mathbf{r})\right\rangle=\epsilon_{p}
\end{gathered}
$$

This results in the following matrix:

$$
\left(\begin{array}{cc}
\epsilon_{p} & t_{k} \\
t_{k}^{*} & \epsilon_{p}
\end{array}\right)
$$

Its eigenvalues lead to the band diagram and is given in Fig. 2.14(b). The difference between both approaches is very substantial. The first approach has flat 


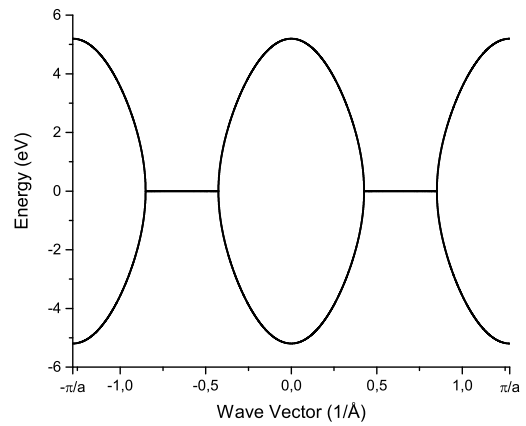

(a)

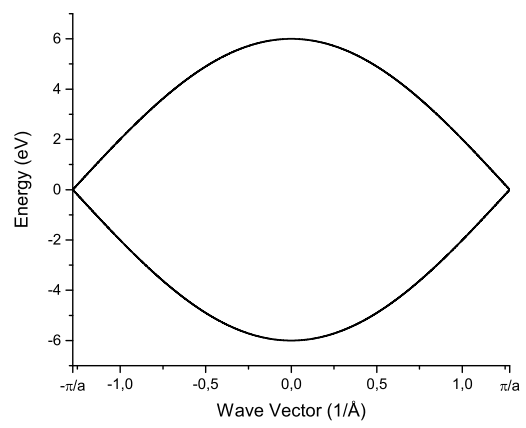

(b)

Figure 2.14 - In panel (a) the band diagram obtained from coupling two individual strips is depicted. The unit cell is twice as large. The band diagram shows flat bands at the Fermi-level. Panel (b) shows the band diagram as acquired from the method in which a unit cell is chosen such that all atoms from the first and second strip are residing in the cell.

bands that will give rise to a huge peak in the density of states at the Fermi-level. This feature is missing in the second approach. The presence of flat bands in the first approach gives more reliability to the first approach. However, the unit cell in the band diagram for the first approach seems to have been cut in half. This is not a surprise, since the unit cell in real space is enlarged twice in order to calculate the first single strip Hamiltonian. In this sense the second approach seems to be more reliable.

\section{Extending zigzag-edge ribbons}

Following the same procedure as for the armchair edges the zigzag nanoribbon tight-binding model is calculated. The illustration of the problem is shown in Fig. 2.13(b,c). Each new strip supplies two atoms in a unit cell. Consequently, the matrix expands with two columns and two rows. If one takes a random atom from the unit cell with the exception of the very edge, then this atom is located either exactly below or above a single atom or coupled to two nearest neighbour atoms with an angle of $120^{\circ}$ in between. Therefore, the coupling matrix elements consist of two kinds. The single atom matrix element is called $H_{0}$ and is defined as $-t\left(e^{-i k_{y} d}\right)$ for an atom coupling to its vertically lower neighbour. The other coupling matrix element (working on two atoms) is labelled as $H_{1}$ and is given by $-t\left(e^{-i k_{x} \frac{d \sqrt{3}}{2}-i k_{y} \frac{d}{2}}+e^{i k_{x} \frac{d \sqrt{3}}{2}-i k_{y} \frac{d}{2}}\right)$. This matrix element is defined such that the two atoms are located below the centred atom.

The smallest width, $\mathrm{N}=2$, contains four atoms as shown in Fig. 2.13(b) and leads 
to the following matrix.

$$
\hat{H}=\left(\begin{array}{llll}
H_{A A} & H_{A B} & H_{A C} & H_{A D} \\
H_{B A} & H_{B B} & H_{B C} & H_{B D} \\
H_{C A} & H_{C B} & H_{C C} & H_{C D} \\
H_{D A} & H_{D B} & H_{D C} & H_{D D}
\end{array}\right)=\left(\begin{array}{cccc}
\epsilon_{p} & H_{1} & 0 & 0 \\
H_{1}^{*} & \epsilon_{p} & H_{0} & 0 \\
0 & H_{0}^{*} & \epsilon_{p} & H_{1} \\
0 & 0 & H_{1}^{*} & \epsilon_{p}
\end{array}\right)
$$

The eigenvalues give the band structure as shown in Fig. 2.15(a). The second smallest strip contains six atoms in the unit cell and is given in Fig. 2.15(b). Its Hamiltonian is a $(6 \times 6)$-matrix:

$$
\hat{H}=\left(\begin{array}{cccccc}
\epsilon_{p} & H_{1} & 0 & 0 & 0 & 0 \\
H_{1}^{*} & \epsilon_{p} & H_{0} & 0 & 0 & 0 \\
0 & H_{0}^{*} & \epsilon_{p} & H_{1} & 0 & 0 \\
0 & 0 & H_{1}^{*} & \epsilon_{p} & H_{0} & 0 \\
0 & 0 & 0 & H_{0}^{*} & \epsilon_{p} & H_{1} \\
0 & 0 & 0 & 0 & H_{1}^{*} & \epsilon_{p}
\end{array}\right)
$$

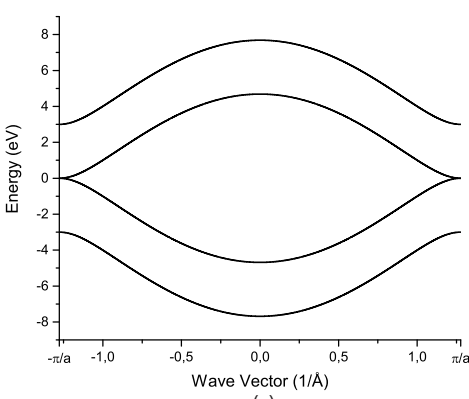

(a)

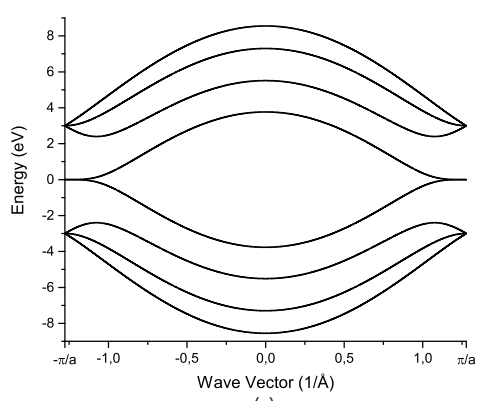

(c)

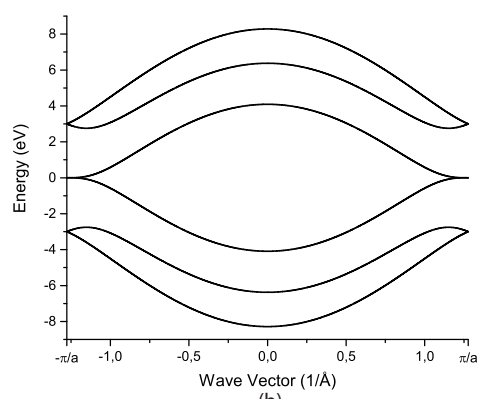

(b)

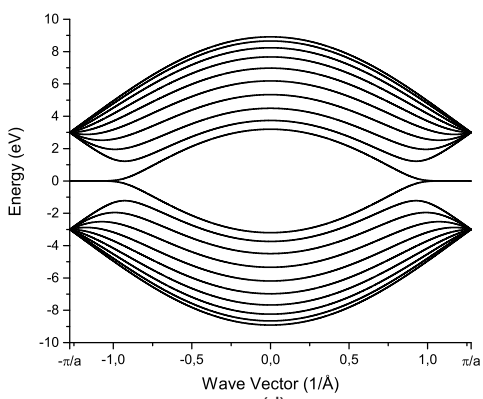

(d)

Figure 2.15 - The band structures of the zigzag graphene nanoribbon with various width, i.e. 2 (a), 3 (b), 4 (c) and 10 (d). Depending on the width of the nanoribbon the flatness of the bands around the Fermi-level increases.

All bands are metallic. 
Each new strip is followed firstly by a coupling via $H_{0}$ and subsequently by $H_{1}$. If the matrix of the $(N-1)^{\text {th }}$-strip is known, the additional matrix elements for the $N^{\text {th }}$-strip problem are acquired as follows

$$
\begin{gathered}
\left\{\begin{array}{l}
H_{2 N-2,2 N-1}=H_{0} \\
H_{2 N-1,2 N-2}=H_{0}^{*}
\end{array}\right. \\
\left\{\begin{array}{l}
H_{2 N-1,2 N}=H_{1} \\
H_{2 N, 2 N-1}=H_{1}^{*}
\end{array}\right. \\
\left\{\begin{array}{l}
H_{2 N-1,2 N-1}=\epsilon_{p} \\
H_{2 N, 2 N}=\epsilon_{p}
\end{array}\right.
\end{gathered}
$$

Eventually the following matrix is acquired.

$$
\left(\begin{array}{cccccc}
\ddots & \ddots & \ddots & \ddots & 0 & 0 \\
\ddots & \ddots & \ddots & \ddots & 0 & 0 \\
\ddots & \ddots & \ddots & \ddots & 0 & 0 \\
\ddots & \ddots & \ddots & \ddots & H_{0} & 0 \\
0 & 0 & 0 & H_{0}^{*} & \epsilon_{p} & H_{1} \\
0 & 0 & 0 & 0 & H_{1}^{*} & \epsilon_{p}
\end{array}\right)
$$

The band structures for $\mathrm{N}=4$ and $\mathrm{N}=10$ as retrieved from this method are shown in Fig. 2.15(c,d). The flatness at the Fermi-level seems to broaden as the width of the nanoribbon increases.

\section{Zigzag edges analysis at Fermi level}

The states around the Fermi level are located around $k= \pm \frac{\pi}{a}$. For $\mathrm{N}=2, \mathrm{~N}=4$ and $\mathrm{N}=8$ in Fig. 2.16 the bands nearby the Fermi level and at the edge of the Brillouin zone are given. It is interesting to see that the 'flatness' of the center bands increases with the width of the nanoribbon. The inset of Fig. 2.16(b) shows the density of states versus energy. At the Fermi level an immense peak in the density of states is present as a result of the flat band that is characteristic for the zigzag nanoribbon. When the flatness is taken into account of all the bands, then there seems to be a maximum of flatness at $N=7$ [?]. With increasing width the edge states become more graphene-like, i.e. the density of states at the Fermi-level decreases.

The highest band of the valence band and the lowest band of the conduction band are degenerate around $k= \pm \frac{\pi}{a}$. If any band structure projections from a true 2Dgraphite was present, the degeneracy would be expected to appear at $k= \pm \frac{2 \pi}{3 a}$ [?]. 
This is clearly not the case, meaning that the electronic states are localized at the edges. The edge states decay towards the middle of the nanoribbon. Such a phenomenon makes the zigzag armchair nanoribbon magnetic. However, upon including spin degrees of freedom and using first-principle calculations a magnetic insulating ground state is found that eventually leads to a band gap, and consequently renders the zigzag nanoribbon to be a semiconductor [?]. Experimental results are needed to confirm which theory is more favorable.

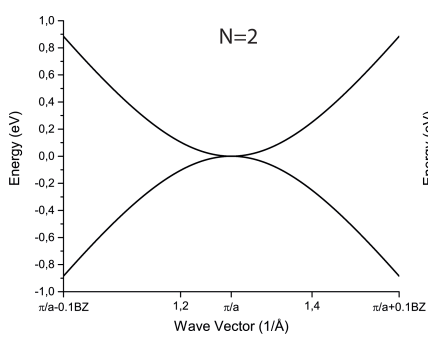

(a)

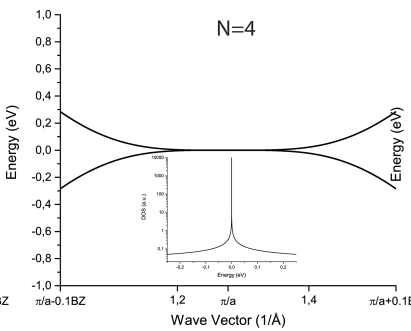

(b)

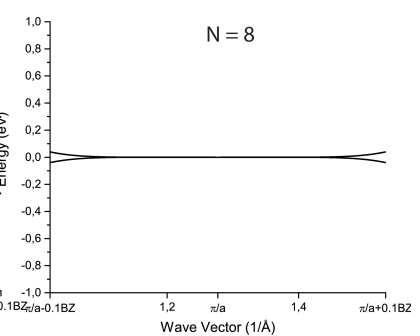

(c)

Figure 2.16 - The band structures in the proximity of the edges of the Brillouin zone with various width, i.e. 2 (a), 4 (b) and 8 (c). The flatness indeed increases by increasing widths. In the inset of (b) the density of states versus energy is given for states around the Fermi-level. A huge spike is observed at the Fermi-level due to the flat bands. 


\subsection{Tight-binding model for hexagonal boron-nitride}

The tight-binding model up to Eq. 2.3 is constructed such that it is applicable for both $\mathrm{h}-\mathrm{BN}$ and graphene. The equation is once again given below, but this time with adapted parameters for the h-BN calculation:

$$
E_{\mathbf{k}}=\frac{\left(\epsilon_{B}+\epsilon_{N}\right) \pm \sqrt{\left(\epsilon_{B}+\epsilon_{N}\right)^{2}-4\left(\epsilon_{B} \epsilon_{N}-t_{\mathbf{k}}^{*} t_{\mathbf{k}}\right)}}{2}
$$

The difference between graphene and $\mathrm{h}-\mathrm{BN}$ lies in the hopping parameter $\mathrm{t}$, the values for $\epsilon_{a}$ and $\epsilon_{b}$ and the lattice-parameter. The latter is in case of h-BN equal to $2.52 \AA$. The special points in reciprocal space are similar to that of graphene. All that is needed is to find the values for these parameters and compute the energy dispersion relation as was done for graphene. The hopping parameter $t$ is $-2.16 \mathrm{eV}$, whereas the valence orbital energies $\epsilon_{B}$ and $\epsilon_{N}$ are $2.46 \mathrm{eV}$ and $-2.55 \mathrm{eV}$ for boron and nitrogen respectively [?]. Figure 2.17 shows the band diagram of hexagonal boron nitride. In this calculation the direct band gap is $5.01 \mathrm{eV}$, which is much smaller than the energy of $5.9 \mathrm{eV}$ found in DFT-calculations [?]. A recent study has shown that the band gap is indeed $5.9 \mathrm{eV}$, but that it is also an indirect band gap [?]. The nearest neighbor tight-binding method is the simplest approximation to get the band diagram. In this calculation only the $p_{z^{-}}$ orbitals were included. No (pseudo)-potentials were used and mixing between molecular bonds was excluded. Nonetheless, the key message in this calculation is the fact that by solely replacing the carbon atoms by boron and nitrogen atoms in a graphene-like structure completely different electronic structure are obtained. On one hand there is graphene with its Dirac-cone and quantumrelativistic behavior, on the other hand there is h-BN being a wide band gap material that is classified as an insulator due to its band gap of $5.01 \mathrm{eV}$ at the K-point.

\subsection{Appendix: Tight-binding method; second quan- tization}

\subsubsection{Introduction}

Another approach to solve the tight-binding problem comes from the second quantization formalism. A general and easy approach will first be given and later on applied to graphene 


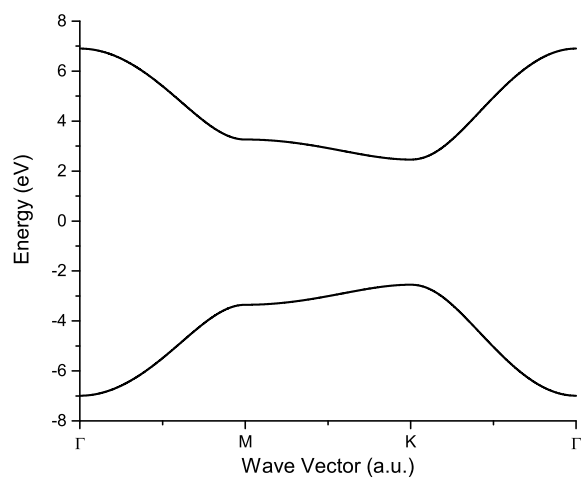

Figure 2.17 - Band structure of h-BN obtained via the tight-binding method. At the K-point the Dirac-cone there is direct band gap of $5.01 \mathrm{eV}$.

$$
\hat{H}=-\sum_{\vec{j}, \vec{l}} t_{\vec{j}, \vec{l}} \hat{c}_{\vec{l}}^{\dagger} \hat{c}_{\vec{j}}
$$

The $t_{j, l}$ is called the hopping parameter. In case of the nearest neighbor tightbinding method the hopping distance from one atom to the nearest atom is constant. Atoms beyond the nearest neighbor approximation will give zero value for $t_{\vec{j}, \vec{l}}$. The operator $\hat{c}_{\vec{l}}^{+}$creates an electron at site $\vec{l}$, while $\hat{c}_{\vec{j}}$ annihilates an electron at site $\vec{j}$. Defining $\vec{j}=\vec{l}+\vec{\tau}$ with $\vec{\tau}$ the nearest neighboring atom(s) to $l$

$$
\hat{H}=-t \sum_{\vec{\tau}, \vec{l}} \hat{c}_{\vec{l}}^{\dagger} \hat{c}_{\vec{l}+\vec{\tau}}
$$

The operators $\hat{c}_{\vec{l}}^{\dagger}$ and $\hat{c}_{\vec{l}+\vec{\tau}}$ have discrete dependence and can be translated to kspace (and thereby continuous dependence) by using the inverse Fourier transform as follows:

$$
\begin{gathered}
\hat{c}_{\vec{l}}^{\dagger}=\frac{1}{\sqrt{N}} \sum_{\vec{q}} e^{-i \vec{q} \cdot r_{l}} \hat{c}_{\vec{q}}^{\dagger} \\
\hat{c}_{\vec{l}+\vec{\tau}}=\frac{1}{\sqrt{N}} \sum_{\vec{k}} e^{i \vec{k} \cdot r_{l}} e^{i \vec{k} \cdot \vec{\tau}} \hat{c}_{\vec{k}}
\end{gathered}
$$

Substituting these new operators in the Hamiltonian gives:

$$
\hat{H}=\frac{-t}{N} \sum_{\vec{\tau}, \vec{l}} \sum_{\vec{k}, \vec{q}} \hat{c}_{\vec{q}}^{\dagger} \hat{c}_{\vec{k}} e^{i r_{l} \cdot(k-\vec{q})} e^{i \vec{k} \cdot \vec{\tau}}
$$


In the nearest neighbor approximation only the term acting on $\vec{l}$ is considered. All other $\vec{l}$ are disregarded. The $\vec{l}$-dependent part of the Hamiltonian can be rewritten as:

$$
\frac{1}{N} \sum_{l} e^{i r_{l} \cdot(\vec{k}-\vec{q})}=\delta_{k q}
$$

Here $\delta_{k q}$ is the Kronicker delta. After applying the Kronicker delta (and with that $\vec{k}=\vec{q}$ ) the Hamiltonian then becomes:

$$
\hat{H}=-t \sum_{\vec{\tau}} \sum_{\vec{k}} \hat{c}_{\vec{k}}^{\dagger} \hat{\vec{c}}_{\vec{k}} e^{i \vec{k} \cdot \vec{\tau}}=\sum_{\vec{k}} E_{\vec{k}} \hat{c}_{\vec{k}}^{\dagger} \hat{c}_{\vec{k}}
$$

From this equation the energy dispersion is given by:

$$
E_{\vec{k}}=-t \sum_{\vec{\tau}} e^{i \vec{k} \cdot \vec{\tau}}
$$

\subsubsection{Applying the second quantization tight-binding method to graphene}

For the sake of convenience the vector-sign is dropped. The Hamiltonian of graphene is given by:

$$
\hat{H}=-t \sum_{R_{i}} \sum_{j=1}^{3}\left(\hat{c}_{A, R_{i}}^{\dagger} \hat{c}_{B, R_{i}+n_{j}}+\hat{c}_{A, R_{i}} \hat{c}_{B, R_{i}+n_{j}}^{\dagger}\right)
$$

The sum $\sum_{j=1}^{3}$ and $n_{j}$ correspond to the nearest neighbor atoms in graphene. The indices A and B are representing the two sublattices. The first term in the summation is annihilating the electron at sublattice B which is located only a neighboring distance away from $R_{i}$ and therefore sublattice A. On the latter sublattice an electron is created. The second term is the Hermitian conjugate and processes the reverse. The operators are like-wise inverse Fourier transformed to k-space:

$$
\left\{\begin{array}{l}
\hat{c}_{A, R_{i}}^{\dagger}=\frac{1}{\sqrt{N}} \sum_{q} e^{-i q \cdot R_{i}} \hat{c}_{A, q}^{\dagger} \\
\hat{c}_{A, R_{i}+n_{j}}=\frac{1}{\sqrt{N}} \sum_{q^{\prime}} e^{i q^{\prime} \cdot R_{i}} e^{i q^{\prime} \cdot n_{j}} \hat{c}_{A, q^{\prime}} \\
\hat{c}_{B, R_{i}}^{\dagger}=\frac{1}{\sqrt{N}} \sum_{k} e^{-i k \cdot R_{i}} \hat{c}_{B, k}^{\dagger} \\
\hat{c}_{B, R_{i}+n_{j}}=\frac{1}{\sqrt{N}} \sum_{k^{\prime}} e^{i k^{\prime} \cdot R_{i}} e^{i k^{\prime} \cdot n_{j}} \hat{c}_{B, k^{\prime}}
\end{array}\right.
$$


The substitution of this set of operators into Eq. 2.44 gives:

$$
\hat{H}=\frac{-t}{N} \sum_{k^{\prime}, q} \sum_{k, q^{\prime}} \sum_{R_{i}} \sum_{j=1}^{3}\left(\hat{c}_{A, q}^{\dagger} \hat{c}_{B, k^{\prime}} e^{i R_{i} \cdot\left(q-k^{\prime}\right)} e^{i k^{\prime} \cdot n_{j}}+\hat{c}_{B, c^{\prime}}^{\dagger} \hat{c}_{A, q^{\prime}} e^{-i R_{i} \cdot\left(q^{\prime}-k\right)} e^{i q^{\prime} \cdot n_{j}}\right)
$$

In analogy with Eq. 2.42 and the fact that the nearest neighbor approximation is involved the following needs to be implemented in Eq. 2.45:

$$
\left\{\begin{array}{l}
\frac{1}{N} \sum_{R_{i}} e^{i R_{i} \cdot\left(q-k^{\prime}\right)}=\delta_{k^{\prime} q} \\
\frac{1}{N} \sum_{R_{i}} e^{-i R_{i} \cdot\left(q^{\prime}-k\right)}=\delta_{k q^{\prime}}
\end{array}\right.
$$

Consequently $k=k^{\prime}=q=q^{\prime}$ and the Hamiltonian becomes:

$$
\hat{H}=-t \sum_{k} \sum_{j=1}^{3}\left(e^{i k \cdot n_{j}} \hat{c}_{A, k}^{\dagger} \hat{c}_{B, k}+e^{-i k \cdot n_{j}} \hat{c}_{B, k}^{\dagger} \hat{c}_{A, k}\right)
$$

Now define $t_{k}$ such that the summation over $j$ disappears and the Hamiltonian becomes easier to interpret:

$$
t_{k}=-t \sum_{j=1}^{3} e^{i k \cdot n_{j}}
$$

Again, this is nothing more than the energies found for hopping from a carbonatom to its three neighboring atoms. The Hamiltonian then reads:

$$
\hat{H}=\sum_{k}\left(t_{k} \hat{c}_{A, k}^{\dagger} \hat{c}_{B, k}+t_{k}^{*} \hat{c}_{B, k}^{\dagger} \hat{c}_{A, k}\right)=\sum_{k} E_{k}
$$

Solving Eq. 2.47 for $E_{k}$ will give the dispersion relation. This is done by introducing the following spinor(s):

$$
\Psi=\left(\begin{array}{l}
\hat{c}_{A, k} \\
\hat{c}_{B, k}
\end{array}\right)
$$

and

$$
\Psi^{*}=\left(\begin{array}{ll}
\hat{c}_{A, k}^{\dagger} & \hat{c}_{B, k}^{\dagger}
\end{array}\right)
$$

One property of the creation and annihilation operators is $\hat{c}_{A, k}^{\dagger} \hat{c}_{A, k}^{\dagger}=1$. Consequently, $\Psi^{*} \Psi=1$. Since $E_{k}$ is a scalar one may rewrite it as: 


$$
E_{k}=\Psi^{*} E_{k} \hat{I}^{22} \Psi
$$

Here $\hat{I}^{22}$ is $2 \times 2$-identity matrix. By using the spinor the argument of the sum on the left-hand side can be transformed to matrices and vectors as well:

$$
\hat{H}=\sum_{k} \Psi^{*}\left(\begin{array}{cc}
0 & t_{k} \\
t_{k}^{*} & 0
\end{array}\right) \Psi=\sum_{k} \Psi^{*}\left(\begin{array}{cc}
E_{k} & 0 \\
0 & E_{k}
\end{array}\right) \Psi
$$

The dispersion relation can be attained by solving the Hamiltonian as given above by computing the determinant:

$$
\left|\begin{array}{cc}
E_{k} & -t_{k} \\
-t_{k}^{*} & E_{k}
\end{array}\right|=0
$$

This equation is familiar and has been solved for the first quantization. It is unnecessary to solve it again here, since it will give the exact same outcome. The hopping parameters $t_{k}$ and $t_{k}^{*}$ are also equal to the ones found in the first quantization method.

$$
E_{k}= \pm \sqrt{t_{k}^{*} t_{k}}= \pm t \sqrt{3+2 \cos \left(\frac{k_{x} a \sqrt{3}}{2}-\frac{k_{y} a}{2}\right)+2 \cos \left(k_{y} a\right)+2 \cos \left(\frac{k_{x} a \sqrt{3}}{2}+\frac{k_{y} a}{2}\right)}
$$




\section{Bibliography}

[1] P.R. Wallace. Phys. Rev., 71(622), 1947.

[2] A.H. Castro Neto, F. Guinea, N.M.R. Peres, K.S. Novoselov, and A.K. Geim. Rev. Mod. Phys., 81:109-154, 2009.

[3] Y. Gui, Z. Yufeng, and Y. Xunwang. Journal of Semiconductors, 34:8, 2013.

[4] J.C. Slater and G.F. Koster. Phys. Rev., 94:6, 1954.

[5] S. Cahangirov, M. Topsakal, E. Akturk, H. Sahin, and S. Ciraci. Phys. Rev. Lett., 102(236804), 2009.

[6] K. Takeda and K. Shiraishi. Phys. Rev. B., 50:14916, 1994.

[7] C-C Liu, W. Feng, and Y. Yao. Phys. Rev. Lett., 107(076802), 2011.

[8] M. Ezawa. New Journal of Physics, 14(033003), 2012.

[9] D. Jose and A. Datta. Journal of Phys. Chem. C., 116:24639-24648, 2012.

[10] H.A. Jahn and E. Teller. Proceddings of the Royal Society A, 161(905):220-265, 1937.

[11] Y. Kubota, K. Watanabe, O. Tsuda, and T. Taniguchi. Science, 317(5840):932934, 2007.

[12] G. Cassabois, P. Valvin, and B. Gil. Nature Photonics, 10:262-266, 2016.

[13] P.A.M. Dirac. Proceedings of the Royal Society A, 117:778, 1928.

[14] K. Nakada, M. Fujita, G. Dresselhaus, and M.S. Dresselhaus. Phys. Rev. B., 54:24, 1996.

[15] L. Brey and H.A. Fertig. Phys. Rev. B., 73:195408, 2006.

[16] A. Cortijo and M.A.H. Vozmediano. Nucl. Phys. B., 763:293, 2007.

[17] M. Ezawa. Phys. Rev. B., 73:045432, 2006.

[18] Y.-W. Son, M.L. Cohen, and S.G. Louie. Phys. Rev. Lett., 97:216803, 2006. 
[19] W.-X. Wang, M. Zhou, X. Li, S.-Y. Li, X. Wu, W. Duan, and L. He. Phys. Rev. B., 93:241403(R), 2016.

[20] Paul Giraud. Study of the electronic structure of hexagonal boron nitride on metals substrates. Master's thesis, Universidad del Pais Vasco, 2012. 


\section{Chapter 3}

\section{The instability of Si on Ag(111)}

\subsection{Introduction}

The first 2D-Dirac material (graphene) was awarded with the Nobel Prize for Physics 2010 and inspired intensively the search for and exploration of other 2D-Dirac materials [?,?,?]. Graphene exhibits a honeycomb structure comprised of $\mathrm{sp}^{2}$-hybridized carbon atoms. Exciting physical properties as Klein tunneling, quantum (spin) Hall effect, anomalous Hall effect and high electron mobilities have been predicted [?,?,?]. In the periodic table of the elements silicon is the lower neighbor of carbon. Silicon comprises a similar electronic configuration $\left([\mathrm{Ne}] 3 \mathrm{~s}^{2} 3 \mathrm{p}^{2}\right)$ as carbon $\left([\mathrm{He}] 2 \mathrm{~s}^{2} 2 \mathrm{p}^{2}\right)$. It is still an open question whether the silicon analogue of graphene, silicene, exhibits similar structural and electronic properties [?]. Computational studies on the structural properties of silicene address that silicene is indeed thermodynamically stable, but is buckled rather than planar like graphene is [?]. In graphene it is well known that the $\pi-$ $\pi^{*}$-mixing originating from the overlap between the out-of-plane $\mathrm{p}_{z}$-orbitals dominates the fascinating electronic properties of graphene. Silicene has a larger $\mathrm{Si}-\mathrm{Si}$ atom distance accounting for less overlap between the out-of-plane $\mathrm{p}_{z^{-}}$ orbitals [?]. This results in relatively weaker $\pi-\pi^{*}$-mixing and is leading to unstable planar silicene structures. By mixing of $\pi$-bonds and (in-plane) $\sigma$-bonds the $\mathrm{sp}^{2} / \mathrm{sp}^{3}$-hybridized silicon structure gets slightly buckled. Fortunately, the buckling of $0.44 \AA$ is calculated to preserve the Dirac cone at the K-point retaining silicene as a 2D-Dirac material like graphene [?,?,?]. Another welcome advantage of silicene, in contrast to graphene, is that the buckling makes it possible to open a band gap by applying a vertical electric field [?]. From an industrial point of 
view exploiting silicene might be profitable since the semiconductor industry is readily and heavily involved in silicon.

In 2012 the first synthesis of silicene was established by depositing silicon on a $\mathrm{Ag}(111)$-substrate [?]. The $\mathrm{Ag}(111)$-substrate was chosen as a substrate due to the suppressed tendency to form a Ag-Si alloy. Moreover, the silicon honeycomb lattice is almost perfectly commensurate with $\operatorname{Ag}(111)$ [?]. Other substrates that have been studied are $\mathrm{ZrB}_{2}(0001)$ [?], $\operatorname{Ir}(111)$ [?], $\mathrm{MoS}_{2}$ [?, ?], highly oriented pyrolitic graphite [?], $\mathrm{Ru}(0001)$ [?], WSe 2 [?] and $h-\mathrm{MoSi}_{2}$ [?]. The most commonly used substrate to grow silicene is $\mathrm{Ag}(111)$. A heteroepitaxial single layer of $\mathrm{Si}$ atoms arranged in a honeycomb lattice, is commonly referred to as "silicene". In this chapter the term silicene is adapted, although the properties of silicene are generally influenced by the interaction with the substrate. The exact nature of reported Dirac like cones in the $\mathrm{Si} / \mathrm{Ag}(111)$ system is still under debate [?,?, ?,?,?,?,?]. A wealth of superstructures of silicene was found for $\mathrm{Si}$ on $\mathrm{Ag}(111)$ as revealed by LEED (Low Energy Electron Diffraction) and STM (Scanning Tunneling Microscopy) [?,?,?,?,?,?] as $(4 \times 4)$ [?,?,?,?,?], $(\sqrt{13} \times \sqrt{13}) R 13.9^{\circ}[$ [?,?,?], $(2 \sqrt{3} \times 2 \sqrt{3}) R 30^{\circ}[?, ?, ?, ?, ?]$ and a rather rare and yet unresolved structure called the T-phase [?]. From STM and LEED measurements the existence of "multilayers silicene", with an overlayer structure $\left(\frac{4}{3} \sqrt{3} \times \frac{4}{3} \sqrt{3}\right) R 30^{\circ}$, has been claimed, and expected exotic properties (e.g. superconductivity) were reported [?, ?, ?, ?, ?]. Please note that all overlayer structures in this chapter are mentioned with respect to the $\mathrm{Ag}(111)$-crystal and thus not with respect to $(1 \times 1)$-silicene.

Silicon growth on $\mathrm{Ag}(111)$ results in lower symmetry and thus leads to vast complexity in the understanding of silicon on $\operatorname{Ag}(111)$ [?]. Several techniques have been used so far, but real time imaging hand in hand with real space and reciprocal space imaging is pretty rare. Here, a Low Energy Electron Microscopy (LEEM) study is presented together with selected area LEED ( $\mu$ LEED) to provide insight in the stability of silicene, the growth and the variety of silicon structures on $\operatorname{Ag}(111)$. 


\subsection{Materials and Methods}

An Elmitec LEEM III microscope [?] operated at a base pressure of $1 \cdot 10^{-10} \mathrm{mbar}$ was used for the experiments. The samples, $\mathrm{Ag}(111)$ single crystals (Surface Preparation Laboratory), were cleaned by sputtering with argon and annealing at $730 \mathrm{~K}$. The deposition of silicon was performed by heating a silicon wafer to approximately $1350 \mathrm{~K}$. The deposition rate was derived from the 1,022 s required to obtain a maximum fractional coverage of $0.96 \mathrm{ML}$ (see Sec. A.1). Assuming a single layer of silicene, we arrive at a rate of $1.65 \cdot 10^{-2}$ Si-atoms per $\mathrm{nm}^{2}$ per s. In view of the potential bilayer character of silicene that is mentioned elsewhere, [?] we emphasize that although a double layer of silicene would double this rate, it will not affect the basic conclusions of this chapter. The standard bright field imaging mode for LEEM was used with various electron energies, field of views and temperatures. The corresponding parameters are given in the captions of each figure, when appropriate. In all LEEM images dark features represent silicon, whereas bright regions are assigned to the clean $\mathrm{Ag}(111)$-substrate. Diffraction experiments were performed by selecting only a fraction of the beam, $1.4 \mu \mathrm{m}$ in diameter, to obtain local diffraction patterns. A summation of patterns as recorded at different energies was performed and is referred to as cumulative $\mu$ LEED pattern. Cumulative $\mu$ LEED patterns have the advantage of displaying as many as possible spots, but it comes with the disadvantage that in accumulated patterns the intensity of the spots cannot be used for analysis. Spot profile analysis was not performed anyway.

\subsection{Si deposition at constant temperature}

The growth of silicene was carried out in LEEM on a clean $\operatorname{Ag}(111)$ substate kept at $542 \mathrm{~K}$. Silicon is deposited by means of direct current heating of a silicon wafer. Initially, the LEEM images only show a flat surface with steps, but no other structural features, see Fig. 3.1(a). The only measurable change that occurs in this early stage of the deposition is a slow, but gradual decrease in the reflected intensity from the Ag surface, hinting at the formation of a dilute background density of silicon adatoms that act as diffuse scatterers. This phenomenon is discussed in more detail in the appendix given in Sec. A.1. After $70 \mathrm{~s}$ an initial layer of Si starts to form. We presume this layer to be one of the various forms of silicene that was observed in other experiments [?,?]. Islands nucleate randomly on the surface, both on terraces and at steps. Over time the islands expand and coalesce, as is shown in panels (c)-(e). Just before reaching a full layer of silicene bright areas increase in size as shown in Fig. 3.1(f-h). The maximum coverage that is reached is $96 \%$. In the subsequent images Fig. 3.1(f-h) the coverage decreases to $34 \%$. This observation hints that a new phase of $\mathrm{Si}$ on $\operatorname{Ag}(111)$ develops, 
which is of a three-dimensional nature since the material that is deposited to form the initial layer is now confined to a much smaller projected area of the surface. Field distortions that occur around these features and that can be made visible when the current of the objective lens of the instrument is varied also hint that the second phase that forms has three-dimensional features, with a lower projected area.
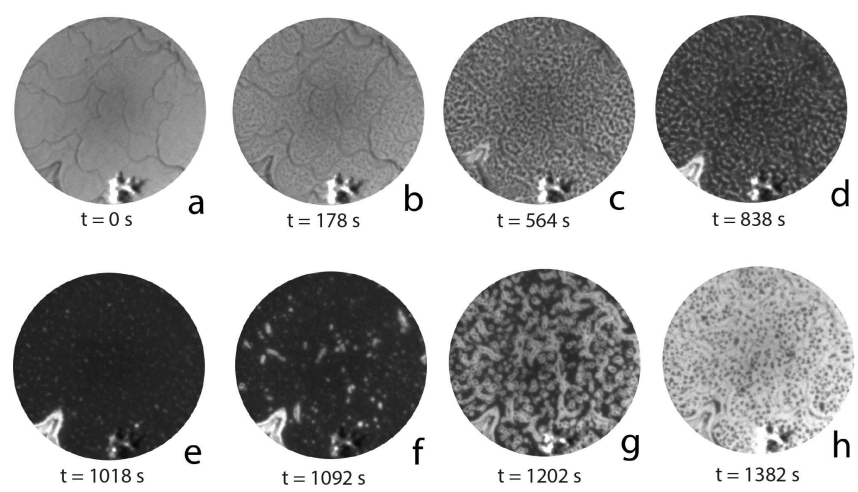

Figure 3.1 - Snapshots of silicon deposition on $\mathrm{Ag}(111)$ recorded with an energy of $18.3 \mathrm{eV}$. FOV $=10 \mu \mathrm{m}$. A characteristic surface feature is observed in the lower left corner. The deposition starts off with a clean $\operatorname{Ag}(111)$ substrate (a). Subsequently, silicene islands nucleate (b) and grow (c-e) till they reach a maximum at $96 \pm 1.5 \%$ (e). The decrease in surface coverage continues (f-g) during deposition until the initial layer is disappeared and only the converted layer is present on the surface $(\mathrm{h})$.

In another experiment with the same conditions the transition to three-dimensional objects was reproduced, while imaged with a larger magnification (Field of view of $2 \mu \mathrm{m})$, see Fig. 3.2. 

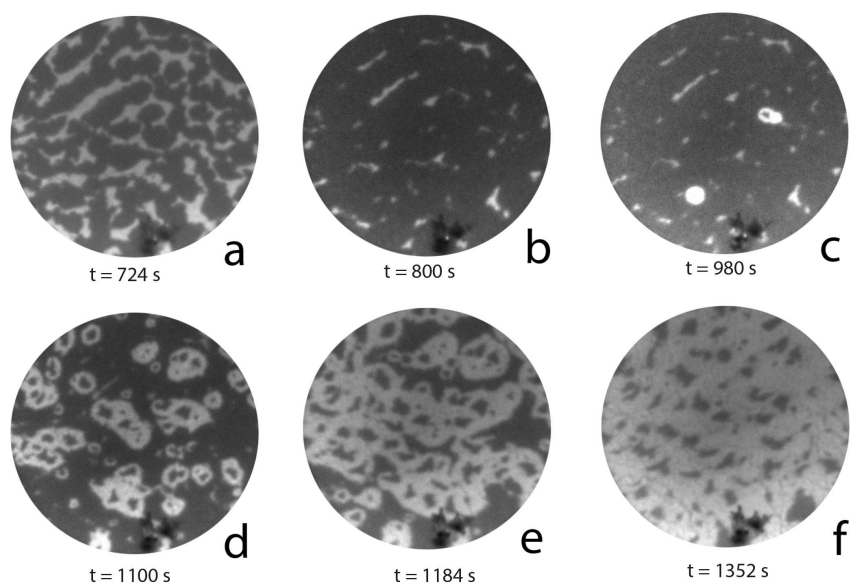

Figure 3.2 - Snapshots of silicon deposition on $\operatorname{Ag}(111)$. FOV $=2 \mu \mathrm{m}$. The temperature was set $539 \mathrm{~K}$ and increased only marginally during the experiment to $544 \mathrm{~K}$. For a detailed description of the observation, see the caption of Fig. 3.1. The area percentages in panels $\mathrm{c}$ and $\mathrm{f}$ are $96.2 \%$ and $12.7 \%$ respectively.

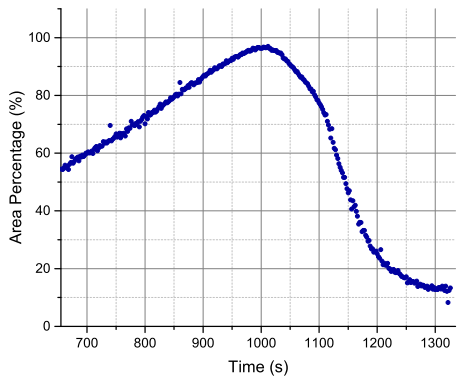

(a)

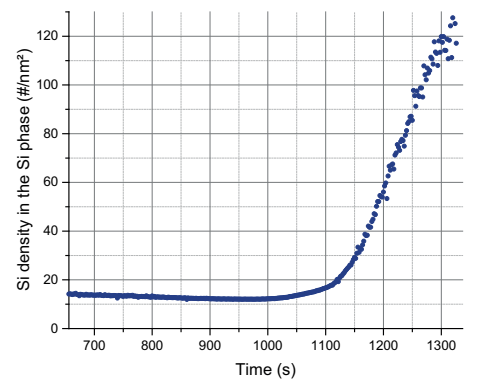

(b)

Figure 3.3 - (a) Area fraction versus time as deduced from the series of Fig. 3.2. The area percentage of silicene does not reach $100 \%$ which demonstrates it is not possible to entirely cover a surface with silicene. Linear increase in area coverage is observed until the maximum of $96.2 \%$ is reached. (b) The Si atom density in the Si phase is fairly constant until the phase transition and suggests a single layer of silicon. After the phase transition the curve increases linearly, which indicates the formation of three-dimensional objects.

The LEEM images shown in Fig. 3.2 were analyzed and the area fraction covered by the Si phases was measured, using a threshold value for the darkness of the Si islands. The area fraction occupied by Si phases is shown in Fig.3.3(a) as a function of time (values before $650 \mathrm{~s}$ are not shown, because experiments there were performed with different FOVs and frequently interrupted for LEED measurements). The area covered by the Si-phases increases linearly in time for 
about $1000 \mathrm{~s}$ as the domains of the initial layer nucleate and grow out. However, full coverage is never reached. The graph peaks at a value of $96 \%$ and continues with a rapid decline, corresponding to the conversion of the initial layer into three-dimensional structures. In Fig. 3.3(b) the Si atom density in the Si phase is shown as function of time (for the extraction of the deposition rate, see Sec A.1).

At times below $\sim 1000 \mathrm{~s}$ the Si atom density in the growing Si phase is fairly constant, indicative of a fixed number of layers (that we assume to be a single layer, as discussed in the next paragraph). After the transition the curve steeply increases, which is indicative of a continuation of the growth in vertical direction, i.e. three-dimensional objects are being formed.

Cumulative $\mu$ LEED-patterns were recorded and are shown in Fig. 3.4. The growth of the initial phase and the corresponding LEED patterns are presented in Fig. $3.4(a, b, c)$. The converted layer is shown in real and reciprocal space in Fig. 3.4(d). The LEED pattern observed for the different coverages of the flat $\mathrm{Si}$ phase in Fig. 3.4 (a-c) are identical and represent a $(2 \sqrt{3} \times 2 \sqrt{3})$ R3 $30^{\circ}$ overlayer structure, see the calculated LEED pattern in Fig. 3.5(a) and the real space model in Fig. 3.5(c). This superstructure is already assigned to a single layer buckled silicon, or silicene, in a number of studies [?,?,?,?,?]. In the experimental data some spots appear double. The reason for this splitting is not settled yet. For different rotational domains the splitting would occur in azimuthal directions and the spacing between the splitted spots would increase with increasing distance from the specular spot. These features are not present. Wang et al. [?] explained the splitting by the formation of a moiré-like pattern, for which they assumed different rotations for 'silicene' spots and ' $2 \sqrt{3}$ ' spots, which is rather unphysical. In addition, a moiré-like pattern around the first order 'silicene' spots is yet not observed, which would be the first position to expect a moiré pattern.

Fig. 3.4(d) shows the converted layer and its diffraction pattern. The following superstructures were found for the converted layer: $\left(\frac{4}{3} \sqrt{3} \times \frac{4}{3} \sqrt{3}\right)$ phase with rotations of $22^{\circ}$ and $38^{\circ}$, see the calculated LEED pattern in Fig. 3.5(b). This overlayer structure is found in other studies as well [?,?,?,?,?]. The nature of this converted layer is still under debate. There are claims that the converted layer is actually bilayer or multilayer silicene [?,?,?]. Shirai et al. reported the formation of a thin film of bulk-like silicon with a terminating $\operatorname{Si}(111)(\sqrt{3} \times \sqrt{3}) R 30^{\circ}-\mathrm{Ag}$ surface [?]. Chen et al. peeled off the surface layer and the $\operatorname{Si}(111)(\sqrt{3} \times \sqrt{3}) R 30^{\circ}$ phase persisted to occur and assigned this phase to a bulk-like Si(111) film [?]. The LEED pattern observed in Fig. 3.4 could very well correspond with a bulklike three dimensional Si-phase. Fig. 3.5(d) displays a possible real space picture for the structure of the converted layer. From the schematic indication of the superstructure in Fig. 3.5(d) it is immediately clear that the structure should be three-dimensional in nature: the left and right $\mathrm{Si}$ atoms in the unit cell are located 
on Ag positions in different layers (a three-fold hollow side in layer $n$, is a on-top side in layer $n+1$ ), indicating that the grown Si structure is most likely threedimensional in nature. The top and bottom Si atoms are very close Ag-positions (in a projected hollow side for three subsequent Ag layers). In the remaining part of this chapter the label $\frac{4}{3} \sqrt{3}$-silicon is used to refer to the converted layer after depositing Si beyond 0.97 ML coverages.

Figure 3.5 (c) reveals that the $(2 \sqrt{3} \times 2 \sqrt{3}) \mathrm{R} 30^{\circ}$ phase contains 14 atoms in the unit cell. The area of the unit cell is 12 times larger than the area of the unit cell of $\operatorname{Ag}(111)$, yielding $\frac{14}{12} \approx 1.16 \mathrm{Si}$ atoms per Ag atom. Each bilayer in Fig. 3.5(d) of silicon accounts for 6 atoms in the unit cell, yielding 12 atoms in the unit cell of which the area is $\frac{16}{3}$ times larger than the $\operatorname{Ag}(111)$ unit cell. For the $\left(\frac{4}{3} \sqrt{3} \times \frac{4}{3} \sqrt{3}\right)$ phase the number of $\mathrm{Si}$ atoms per $\mathrm{Ag}$ atom is thus $\frac{9}{4}=2.25$. The deposition rate of $\mathrm{Si}$ is calibrated using the slope on the left-hand side of Fig.3.3(a). With this calibration value we find that in Fig. 3.2(f) the averaged height of the Si structures would correspond to about 11 bilayers of $\mathrm{Si}$.

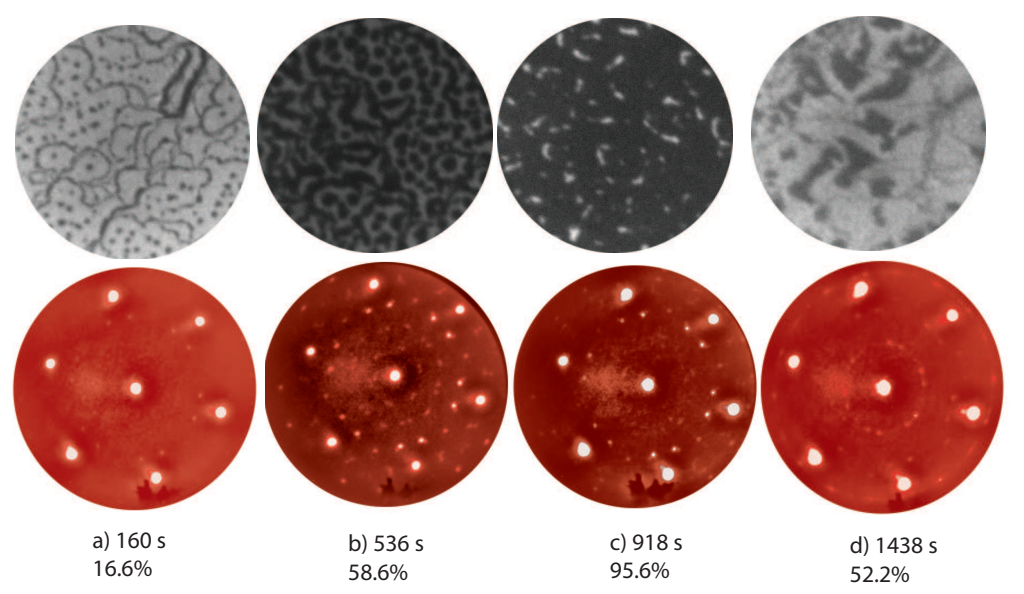

Figure 3.4 - Cumulative LEED patterns were recorded at given coverages during growth. The LEEM images have a field of view of $2.4 \mu \mathrm{m}$. The coverages shown here are $16.6 \%$ (a), 58.6\% (b), 95.6\% (c) and $52.2 \%(\mathrm{~d})$. $(2 \sqrt{3} \times 2 \sqrt{3}) \mathrm{R} 30^{\circ}$ is observed in (a-c). Panel (d) shows different rotations of $\left(\frac{4}{3} \sqrt{3} \times \frac{4}{3} \sqrt{3}\right)$.

\subsubsection{Lower growth temperature: different silicene phase}

In literature different superstructures were found for the silicene layer, namely a mixed phase of $(4 \times 4)$ and the $(\sqrt{13} \times \sqrt{13}) R \pm 13.9^{\circ}[?, ?, ?, ?, ?]$. It was reported that this mixed phase occurred at a lower growth temperature with respect to the $(2 \sqrt{3} \times 2 \sqrt{3}) \mathrm{R} 30^{\circ}$ silicene phase. The phase transition that is addressed in Sec. 3.3 inspired us to deposit silicon on $\mathrm{Ag}(111)$ at a relatively low temperature 


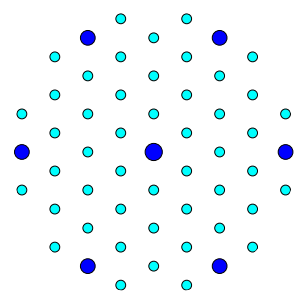

(a)

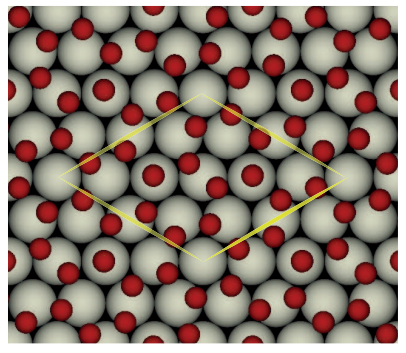

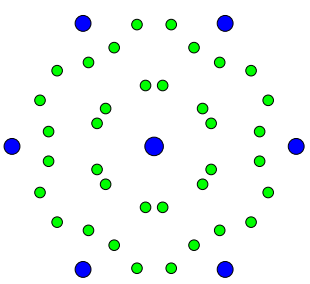

(b)

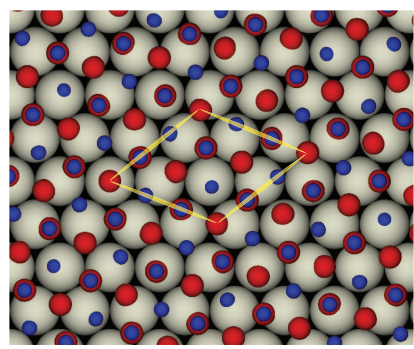

(d)

Figure 3.5 - Simulated LEED patterns of $(2 \sqrt{3} \times 2 \sqrt{3})$ R30 $0^{\circ}$ silicene phase (a) and the $\left(\frac{4}{3} \sqrt{3} \times \frac{4}{3} \sqrt{3}\right) R[22,38]^{\circ}$ bulk-like silicon phase (b). The $\operatorname{Ag}(111)$ spots are depicted as the dark blue spots. The real space model of the silicene phase is given in panel (c) and only the real space model of the $22^{\circ}$-rotational domain of the bulk-like silicon phase is shown in panel (d). The first and the second bilayer of the bulk-Si(111) film are given in red and blue respectively.

in order to verify whether a similar phase transition holds for the mixed phase as well. The growth was conducted at a temperature of $475 \mathrm{~K}$. The image series of the growth is given in Fig. 3.6.

During the growth a cumulative LEED pattern was recorded on both the initial and the converted layer as is shown in Fig. 3.7. The initial phase of silicene is now a mixed phase consisting of $(4 \times 4)$ and $(\sqrt{13} \times \sqrt{13})$ R13.9 $9^{\circ}$ overlayer structures. However, the converted layer still corresponds with the $\left(\frac{4}{3} \sqrt{3} \times \frac{4}{3} \sqrt{3}\right)$-structures as was previously found at $542 \mathrm{~K}$. 


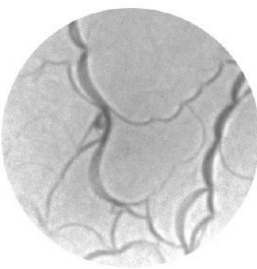

(a) $0 \mathrm{~s}$

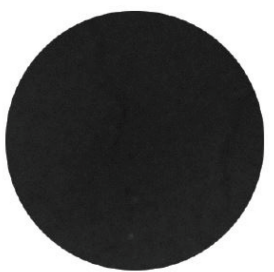

(e) $1063 \mathrm{~s}$

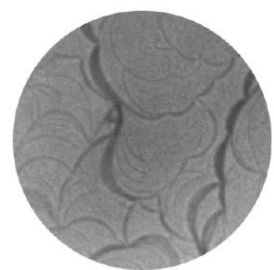

(b) $272 \mathrm{~s}$

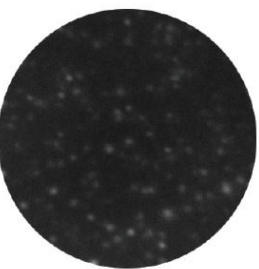

(f) $1441 \mathrm{~s}$

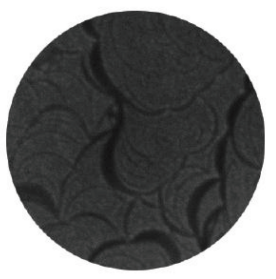

(c) $660 \mathrm{~s}$

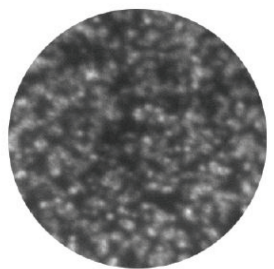

(g) $1815 \mathrm{~s}$

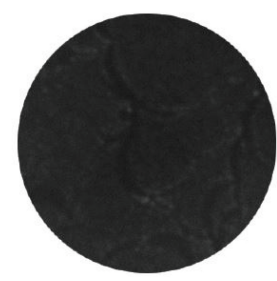

(d) $900 \mathrm{~s}$

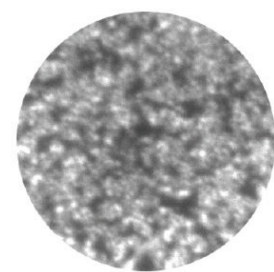

(h) $2153 \mathrm{~s}$

Figure 3.6 - FOV $=6 \mu \mathrm{m}$. The deposition of silicon was conducted at $475 \pm 5 \mathrm{~K}$ and a start voltage of $17.9 \mathrm{eV}$. The size of silicene islands are too small to be resolved by LEEM. Panels (a-e) show the silicene phase, whereas panels $(\mathrm{f}, \mathrm{h})$ contain the converted layer phase of silicon.
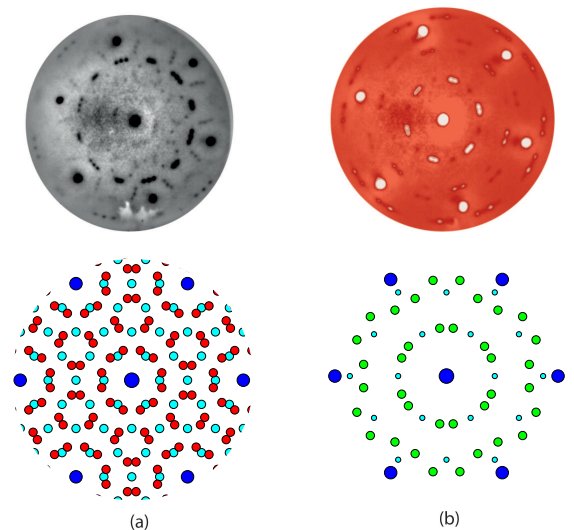

(b)

Figure 3.7 - Cumulative $\mu$ LEED pattern on silicene before the phase transition. The corresponding overlayer structure is a mix of $(4 \times 4)$ (cyan) and $(\sqrt{13} \times \sqrt{13})$ R13.9 $\left(\right.$ red) $\left(\right.$ a). After the phase transition the $\left(\frac{4}{3} \sqrt{3} \times \frac{4}{3} \sqrt{3}\right)$ domains are found by cumulative $\mu$ LEED (b). The large green spots are more dominant and are the rotational domains of this phase. The smaller cyan spot depicts the $\left(\frac{4}{3} \sqrt{3} \times \frac{4}{3} \sqrt{3}\right)$ without rotation.

\subsection{Annealing silicene: new overlayer structure}

The $(2 \sqrt{3} \times 2 \sqrt{3}) \mathrm{R} 30^{\circ}$ silicene phase was grown at a temperature of $553 \mathrm{~K}$ on a freshly cleaned sample until a coverage of $0.5 \mathrm{ML}$ was reached. At this coverage 
the sample was annealed to $629 \mathrm{~K}$ and kept stable at this temperature. The experimental observation is shown in Fig. 3.8. An object appears that consumes all the surrounding silicene islands until no more silicene is present. Around this new nucleus the silicene domains disappear rapidly, whilst the new nucleus expands only marginally. In Fig. 3.8(f) the surface is free of silicene domains, and only the nuclei that formed during the conversion process are left. This process seems very similar to the conversion process described in Sec. 3.3, namely a phase transition from silicene to a three dimensional silicon structure. A $\mu$ LEED pattern of a nucleus similar to the one that is seen in Fig. 3.8(f) is given in Fig. 3.9(a).

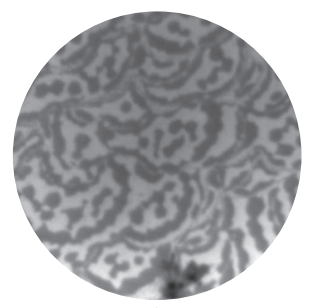

a) $350.6^{\circ} \mathrm{C}-0 \mathrm{~s}$

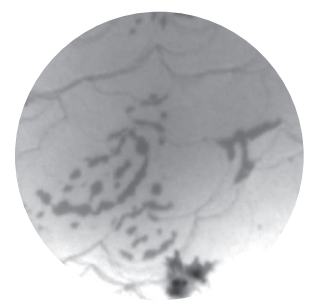

d) $359.0^{\circ} \mathrm{C}-72 \mathrm{~s}$

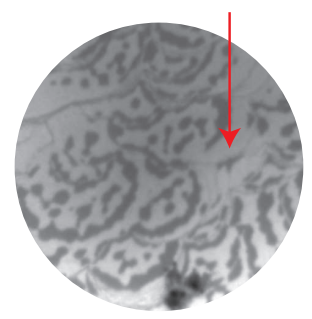

b) $355.3^{\circ} \mathrm{C}-32 \mathrm{~s}$

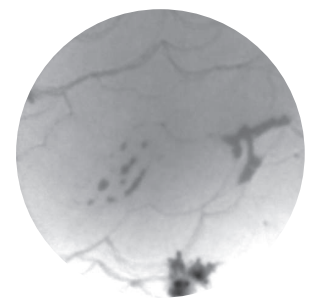

e) $360.7^{\circ} \mathrm{C}-90 \mathrm{~s}$

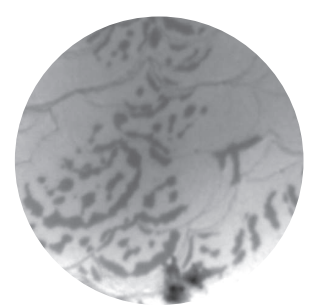

c) $357.5^{\circ} \mathrm{C}-52 \mathrm{~s}$

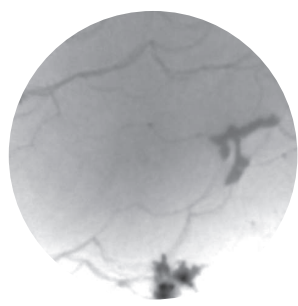

f) $362.2^{\circ} \mathrm{C}-102 \mathrm{~s}$

Figure 3.8 - Conversion of the $(2 \sqrt{3} \times 2 \sqrt{3}) \mathrm{R} 30^{\circ}$ silicene phase. FOV $=4 \mu \mathrm{m}$ and $\mathrm{E}=18.3 \mathrm{eV}$. Darker domains in panel (a) are assigned to silicene exhibiting the $(2 \sqrt{3} \times 2 \sqrt{3}) \mathrm{R} 30^{\circ}$ phase. The silicene layer converts into another phase, which emergence is indicated by the red arrow in panel (b). A similar region develops on the left side of the image, with the nucleus being just outside the field of view.

To perform the LEED measurement the temperature of the sample was reduced to room temperature, in order to prevent that the three dimensional silicon structure would evaporate and/or intercalate. Two rotational domains of $\left(\frac{1}{2} \sqrt{21} \times \frac{1}{2} \sqrt{21}\right)$ with a rotational splitting of $10.9^{\circ}$ are revealed in the LEED pattern. Double spots in the pattern are now due to a rotation of the silicon overlayer structure relative to the substrate. The rotation angle is approximately $10.9^{\circ}$. A $\left(\frac{1}{2} \sqrt{21} \times\right.$ $\left.\frac{1}{2} \sqrt{21}\right) R 10.9^{\circ}$ was also confirmed by simulations as given in Fig. 3.9(b). In matrix notation the structure is given by

$$
\left(\begin{array}{cc}
2 & \frac{1}{2} \\
-\frac{1}{2} & \frac{5}{2}
\end{array}\right)
$$


From the determinant of this superstructure matrix the area of the superstructure unit cell is found to be 5.25 times the area of a $\operatorname{Ag}(111)$ surface unit cell. With 3 $\mathrm{Si}$ atoms in the superstructure unit cell (see Fig. 3.9(c)) a single Si atom occupies $1.75 \mathrm{Ag}(111)$ surface unit cells. This value is very close to

$$
\left(\frac{a_{S i}}{a_{A g}}\right)^{2}=\left(\frac{5.431}{4.090}\right)^{2}=1.763
$$

where $a_{S i}$ and $a_{A g}$ are the bulk lattice parameters of silicon and silver. The deviation is only $0.74 \%$ from 1.763 which we take as evidence for the formation of a bulk silicon structure that is $s p^{3}$ hybridized on top of silver. Again, the area fraction of $\mathrm{Si}$ decreases as a phase transition occurs. This hints on the formation of three-dimensional structures.

Note that within a unit cell of Fig. 3.9(c) one Si-atom is located in a hollow site while three Si-atoms are located on near-bridge sites. This arrangement of the atoms gives strong coupling with the substrate, which is needed to saturate the otherwise dangling bonds of the silicon structure. In conclusion, the three dimensional structure that has been found corresponds to $s p^{3}$ hybridized silicon and thus a transition from $s p^{2} / s p^{3}$ hybridized silicon (silicene) to $s p^{3}$ hybridized silicon was observed.

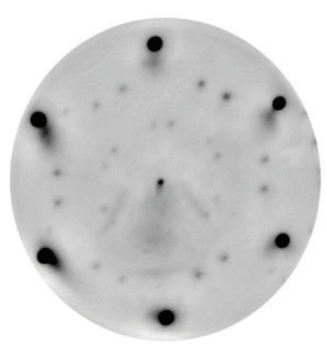

(a)

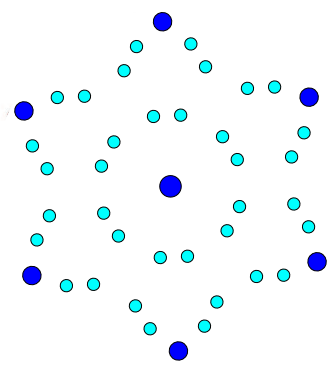

(b)

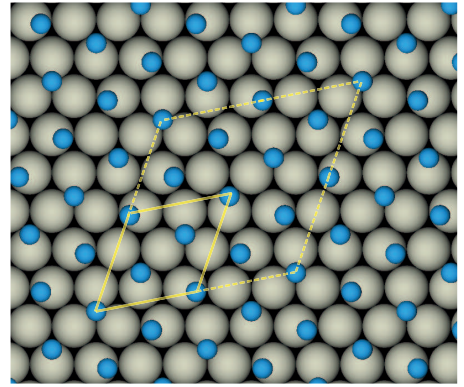

(c)

Figure 3.9 - The measured $\left(\frac{1}{2} \sqrt{21} \times \frac{1}{2} \sqrt{21}\right) R 10.9^{\circ}$ bulk-like silicon phase (a) and its simulated pattern (b) where the cyan spots are the $\left(\frac{1}{2} \sqrt{21} \times\right.$ $\left.\frac{1}{2} \sqrt{21}\right) R 10.9^{\circ}$ spots. A possible real space model of the structure is given in (c). 
Figure 3.8 shows that the conversion from partly $s p^{2}$ to $s p^{3}$ hybridized silicon on $\mathrm{Ag}(111)$ occurs in a very peculiar fashion. Once the conversion temperature has been reached, silicene islands shrink in area at the expense of a three dimensional silicon object as can be seen in the sequence in Fig. 3.10(a-c) for a larger field. The growth in area of a denuded zone around the $s p^{3}$ hybridized silicon was measured by drawing manually a circumference around the zone using ImageJ resulting in an area versus time plot given in Fig. 3.10(d). t Figure 3.10(d) shows

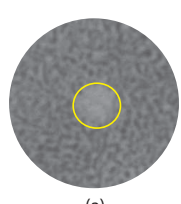

(a)

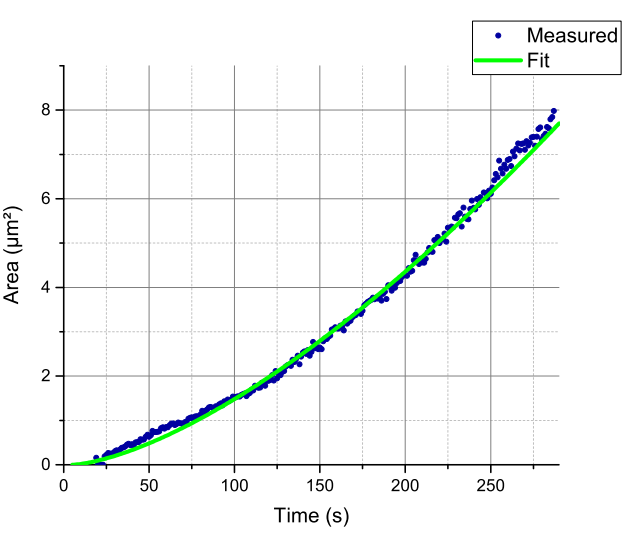

(d)

Figure $3.10-\mathrm{T}=638 \pm 5 \mathrm{~K}, \mathrm{E}=17.9 \mathrm{eV}$ and $\mathrm{FOV}=4.7 \mu \mathrm{m}$. Upon annealing silicene on $\operatorname{Ag}(111)$ a diamond-silicon object grows at the expense of silicene islands $(a-c)$. Time after reaching the desired temperature for panels $(a),(b)$ and (c) is $47 \mathrm{~s}, 211 \mathrm{~s}$ and $284 \mathrm{~s}$ respectively. The area versus time diagram of the denuded zone is given in panel $(\mathrm{d})$ showing non-linear characteristic. Fitting was done by using the coefficients $c_{1}=0.0016$ and $c_{2}=5$ for

$$
A=c_{1}\left(t-c_{2}\right)^{3 / 2} \text {. }
$$

that the area of the denuded zone, $A$, does not increase linearly in time, but exhibits a $A \propto t^{3 / 2}$ time dependence. This means that the area of the silicene phase decreases with $A \propto t^{2 / 3}$. Such behavior is typically the case for diffusion limited kinetics [?]. More evidence is provided when a line profile is drawn as shown in Fig. 3.11. It is clear that the intensity increases towards to the center of the denuded zone implying that there is a larger concentration of diffuse scatterers at the edge of the denuded zone relatively to the more inward areas. The difference in the concentration of diffuse scatterers indicates a concentration gradient, in line with diffusion limited kinetics. 


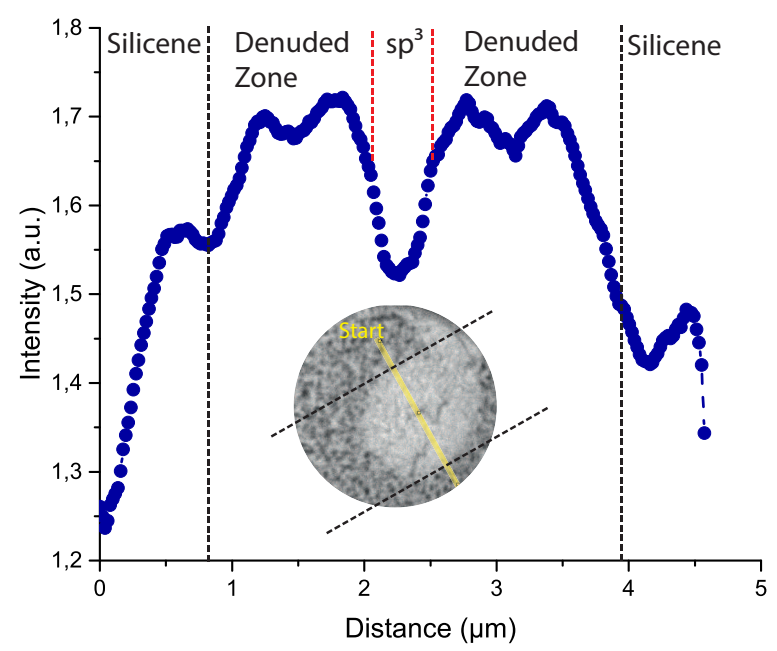

Figure $3.11-\mathrm{T}=638 \mathrm{~K}, \mathrm{E}=17.9 \mathrm{eV}$ and $\mathrm{FOV}=5 \mu \mathrm{m}$. A line profile was drawn on a intensity-inhomogeneity corrected image giving evidence to the presence of a radial gradient in intensity in the denuded zone. Time of measurement was $333 \mathrm{~s}$ after the first glimpse of the denuded zone appeared during the images.

\subsection{X-ray Photoelectron Spectroscopy}

Two different $\operatorname{Ag}(111)$-crystals were used for the growth of silicene. After the silicene growth experiments, the second sample was found to be contaminated with tellurium as X-ray Photoelectron Spectroscopy (XPS) revealed after the silicene growth experiments. The spectrum is given in Fig.3.12 and shows a relatively high dose of contamination of Te with respect to the amount of silicon on the silver substrate. Oxygen and carbon levels were expected to be high since the sample was transferred from the LEEM to the XPS in ambient conditions and no further treatment was performed on the sample. The origin of the contamination remains unclear. An attempt to grow $\mathrm{Bi}_{2} \mathrm{Te}_{3}$ in the setup might have contaminated the sample. Another possible contamination might be related to present tellurium in the crystal upon purchase as was privately discussed with the supplier SPL. In this chapter experiments mentioned in Sections 3.3.1 has been performed on the contaminated sample. 


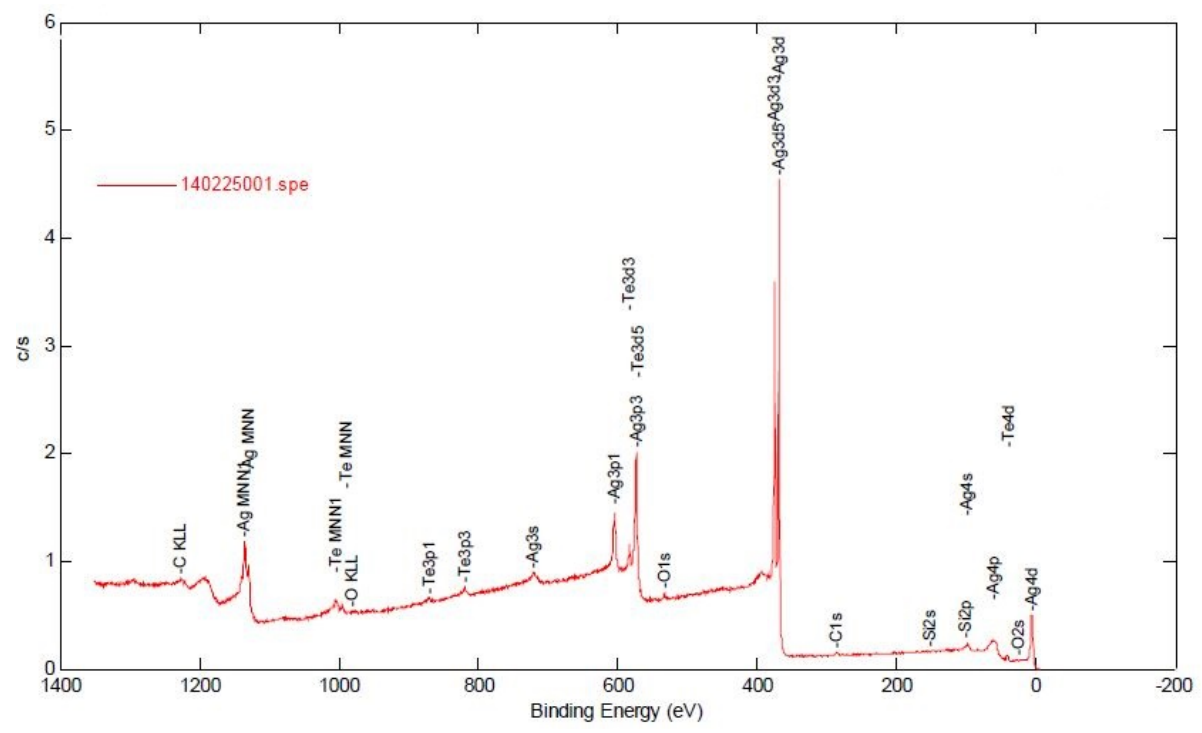

Figure 3.12 - An XPS-study of the sample that was provided to the XPSservice group. The presence of a considerable amount of Te relatively to $\mathrm{Si}$ is evidence of a contaminated sample.

\subsection{Conclusion}

In summary, the growth of silicene on $\operatorname{Ag}(111)$ was studied in-situ with LEEM and $\mu$ LEED. While silicene islands nucleate and grow during the initial stages of the deposition of silicon, a phase transition occurs from partly $s p^{2}$ to "sp $s p^{3}$ like" hybridized silicon before the surface can be fully covered by silicene. The same phase transition can be induced by increasing the substrate temperature, thereby raising the equilibrium density of silicon adatoms on top of the silicene layers. The occurrence of this change in hybridization makes the controlled fabrication of single layer, closed silicene sheets problematic, at least when using Ag substrates, which are commonly considered ideal due to their minute misfit. Either the deposition process itself or the adatoms originating from the silicene domains themselves induce the irreversible structural transition and cause the silicene to self-destruct. Our results further demonstrate that, whereas theoretical considerations predict that the pure form of silicene is energetically stable, its actual, practical stability can only be appropriately judged by considering the environment, e.g. temperature, coverage and substrate, that the silicene is in. This implies that coupling of the silicene layer to the substrate, and its interaction with adatoms may crucially influence the delicate stability of the silicene epitaxial layer, much more so than for graphene, and need to be taken into account. Other, more complex multilayer silicene structures, which were not observed in the present study, are anticipated to be even more susceptible to the observed 

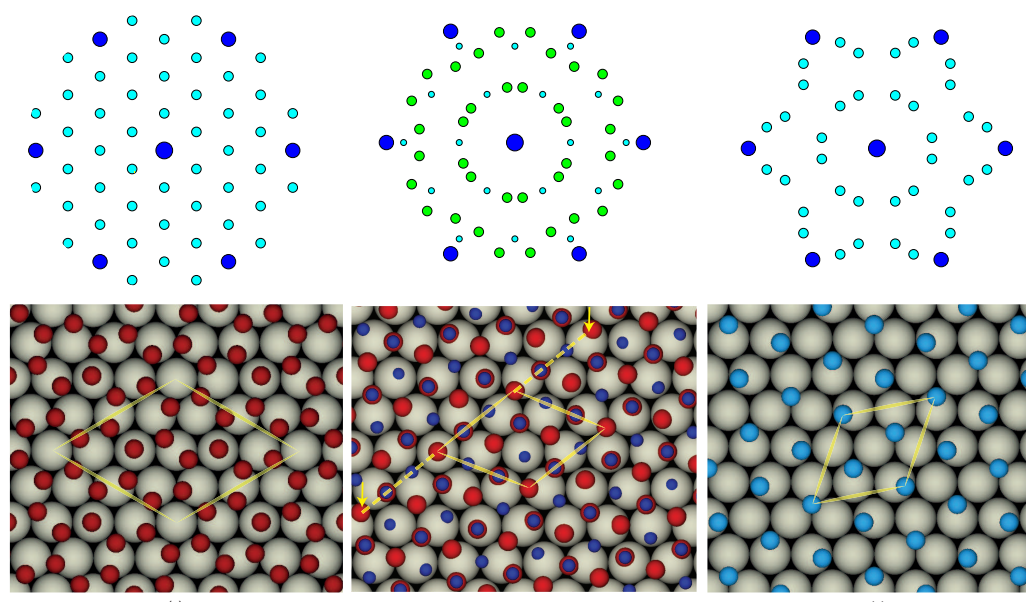

(b)

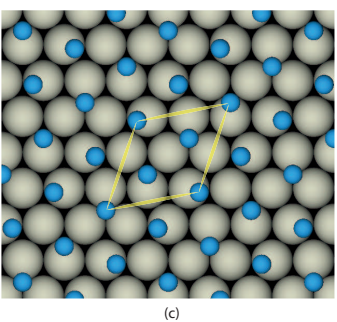

Figure 3.13 - A recap of the structures that were found for silicene. In panel (a) the $(2 \sqrt{3} \times 2 \sqrt{3}) \mathrm{R} 30^{\circ}$ phase is given and corresponds with single layer silicene. Panel (b) depicts the $\left(\frac{4}{3} \sqrt{3} \times \frac{4}{3} \sqrt{3}\right) R 22^{\circ}$ phase, which is one of the two bulk-like Si structures that were found. One direction of the lattice base vectors is extended to illustrate that every three lattice base vector lengths the relative position of a Si-atom projected on the $\mathrm{Ag}(111)$ substrate is repeated. The second bulk-like $\mathrm{Si}$ structure is given in panel (c) and is the $\left(\frac{1}{2} \sqrt{21} \times \frac{1}{2} \sqrt{21}\right) R 10.9^{\circ}$ phase.

instability. 



\section{Appendix A}

\section{A.1 Supersaturation potential retrieval}

From Fig. 3.1 the area fraction versus time plot (Fig. A.1) was acquired.

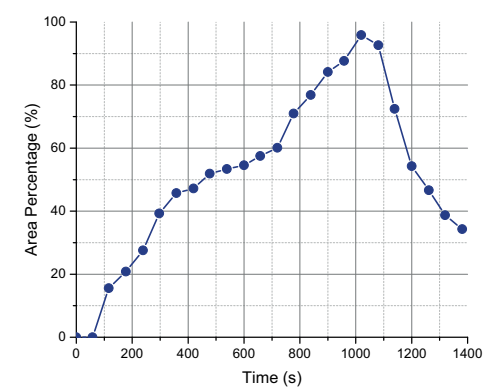

(a)

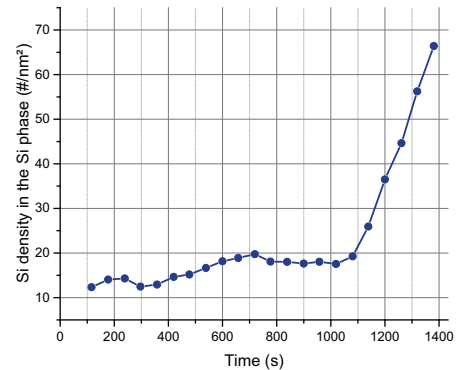

(b)

Figure A.1 - (a) Area fraction versus time as deduced from the series of Fig. 3.1. The area percentage of silicene does not reach $100 \%$ which demonstrates it is not possible to entirely cover a surface with silicene. Silicene islands start to appear after approximately 70 seconds. Afterwards a linear increase of $1.025 \cdot 10^{-3} \mathrm{~s}^{-1}$ is observed until the maximum of $95.7 \%$ is reached. (b) The number of Si-atoms per unit area is fairly constant until the phase transition occurs suggesting a single layer of silicon. After the phase transition the curve increases, indicating the formation of threedimensional objects.

During the first $70 \mathrm{~s}$ of silicon exposure not much seems to happen. This is due to an increase in the amount of diffuse scattering from the substrate prior to the nucleation of any islands. [?,?,?] This indicates the formation of a lattice gas of mobile silicon monomers (or multimers). When the lattice gas density becomes supersaturated, silicon islands nucleate and grow. A quantitative evaluation of the growth process requires knowledge of the amount of silicon that is present on the surface at any moment in time. The deposition rate of silicon was determined 
by finding the superstructure symmetry and coverage using $\mu$ LEED, and using this information to express the area percentage increase over time in a Si coverage. The area fraction increases $1.025 \cdot 10^{-3} \mathrm{~s}^{-1}$. This value was found by least-squares fitting a line to the data shown in Fig. A.1. The superstructure phase exhibiting on top of the substrate was $(2 \sqrt{3} \times 2 \sqrt{3}) \mathrm{R} 30^{\circ}$. The overlayer structure is given by

$$
\left(\begin{array}{ll}
4 & 2 \\
2 & 4
\end{array}\right)
$$

The deposition rate is then calculated by

$$
d=\frac{N \tau}{\Pi A_{A g}}=\frac{14 \cdot 1.025 \cdot 10^{-3}\left(\mathrm{~s}^{-1}\right)}{12 \cdot 0.0724\left(\mathrm{~nm}^{2}\right)}=1.65 \cdot 10^{-2} \mathrm{~nm}^{-2} \mathrm{~s}^{-1}
$$

Here, $d$ is the deposition rate (number of adatoms $/ \mathrm{nm}^{2} / \mathrm{s}$ ), $\tau$ is the increase in area percentage in time obtained from Fig. A.1. $N$ is the number of atoms in one unit cell of silicene, which is 14 . The determinant of the overlayer structure matrix, which is 12 , is given by $\Pi$. $A_{A g}$ is the area of a $\mathrm{Ag}(111)$ unit cell, which is $\frac{1}{4} \sqrt{3} a^{2}$ with $a$ the lattice parameter of silver being $4.090 \AA$.

During the first 70 seconds the intensity of the $\operatorname{Ag}(111)$ substrate changes due to diffuse scattering. The intensity versus time measurement is depicted in Fig. A.2(a). By multiplying Eq. A.1 with time, the intensity is plotted versus the density of silicon adatoms per square nanometer as shown in Fig. A.2(b). The intensity is then calibrated with respect to the density of adatoms by a simple linear fit as done in Fig. A.2(b). Combining this calibration with data from Fig. A.2(a) enables plotting density versus time as in Fig. A.2(c). From this plot the surplus chemical potential beyond equilibrium at the moment that nucleation sets in can be evaluated by using Eq. A.2.

$$
b \Delta \mu=k T \ln \frac{\Theta_{\max }}{\Theta_{e q}}
$$

Here $k$ is the Boltzmann constant which is $8.617 \cdot 10^{-2} \mathrm{meV} \mathrm{K}^{-1} . T$ is the temperature $(546 \mathrm{~K})$. The quantities $\Theta_{\max }\left(0.89 \mathrm{~nm}^{-2}\right)$ and $\Theta_{e q}\left(0.71 \mathrm{~nm}^{-2}\right)$ correspond with the maximum density $\Theta_{\max }$ and the saturation/equilibrium density $\Theta_{e q}$ found in Fig. A.2(c). Equation A.2 is applicable if the dilute phase is assumed to be an ideal two dimensional monatomic lattice gas. Using these values in Eq. A.2 leads to a supersaturation potential $\Delta \mu$ of $9.6 \mathrm{meV}$. To compare, in another experiment performed with the same LEEM used in our experiment, 4, 4'-biphenyldicarboxylic acid (BDA) molecules were deposited on $\mathrm{Cu}(001)$ and a supersaturation of $6 \mathrm{meV}$ was found for nucleation [?]. The supersaturation that is needed to achieve nucleation roughly provides a measure for the energetic stability of the structure that forms. The higher the supersaturation, the lower the energetic stability of 
the phase that forms. Silicene is a relatively unfavorable configuration of silicon. Therefore, because of its lower coordination in the $s p^{2} / s p^{3}$ hybridized states, the fact that the required supersaturation is 1.5 times as high need not be surprising. Repeating the experiment at different temperatures would open the doors to a rich field of thermodynamical analysis. According to Eq. A.2 decreasing the temperature results in a linearly decreasing supersaturation potential difference and thus, in principle, reduces the time needed to form silicene. An interesting analysis would then be to compute supersaturation levels versus the superstructure symmetries in a phase diagram. 
Chapter A.

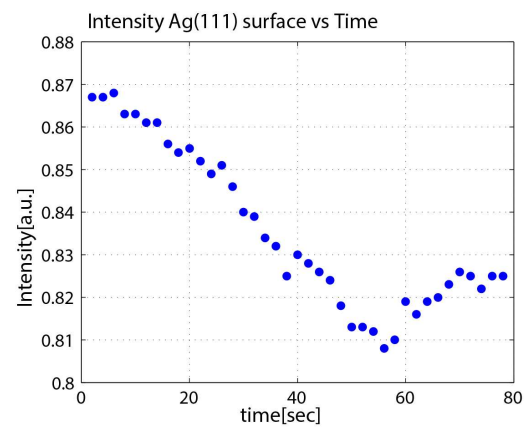

(a)
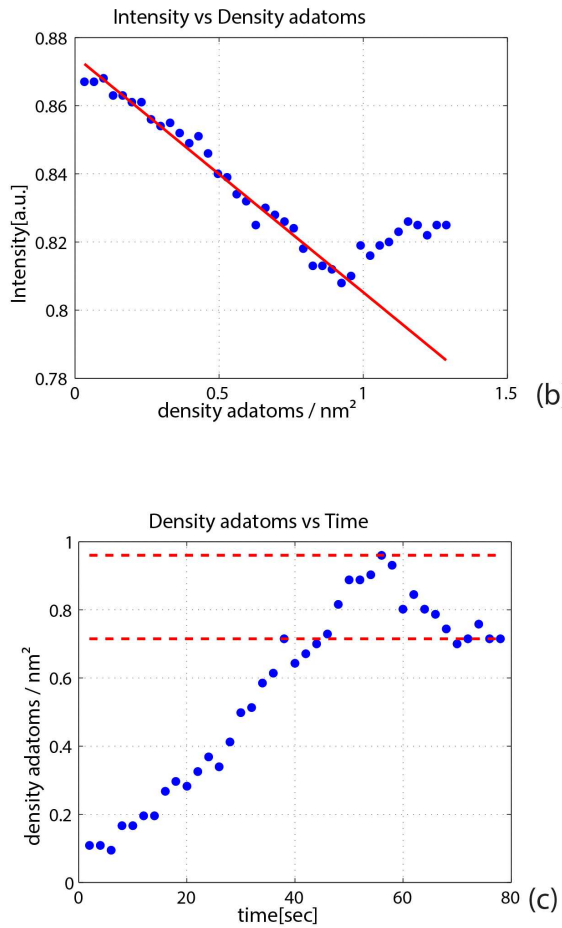

Figure A.2 - From $t=0 s$ to $46 \mathrm{~s}$ the system is as a lattice gas. In between $\mathrm{t}=46 \mathrm{~s}$ and $70 \mathrm{~s}$ supersaturation and nucleation take place. From $\mathrm{t}=70 \mathrm{~s}$ and on the system is in equilibrium $(\mathrm{a}, \mathrm{c})$. From the calibration of intensity with the density of adatoms (b) a density of adatoms vs time graph was plotted containing the $\Theta_{\max }\left(0.89 \mathrm{~nm}^{-2}\right)$ and $\Theta_{\ell q}\left(0.71 \mathrm{~nm}^{-2}\right)$ (c). 


\section{Bibliography}

[1] K.S. Novoselov, A.K. Geim, S.V. Morozov, D. Jiang, Y. Zhang, S.V. Dubonov, I.V. Grigorieva, and R.E. Smalley. Science, 306:666-669, 2004.

[2] M. Xu, T. Liang, S. Minmin, and H. Chen. Chem. Rev., 113:3766-3798, 2013.

[3] T.O. Wehling, A.M. Black-Schaffer, and A.V. Balatsky. Advances in Physics, 63(1):1-76, 2014.

[4] C.L. Kane and E.J. Mele. Phys. Rev. Lett., 95:146802, 2005.

[5] Y. Zhang, Y.-W. Tan, H.L. Stormer, and P. Kim. Nature, 438:201-204, 2005.

[6] A.H. Castro Neto, F. Guinea, N.M.R. Peres, K.S. Novoselov, and A.K. Geim. Rev. Mod. Phys., 81:1, 2009.

[7] K. Takeda and K. Shiraishi. Phys. Rev. B., 50:14916, 1994.

[8] S. Cahangirov, M. Topsakal, E. Aktürk, H. Şahin, and S. Ciraci. Phys. Rev. Lett., 102:236804, 2009.

[9] E.F. Sheka. Int. J. Quantum Chem., 113:612-618, 2013.

[10] C.-C. Liu, W. Feng, and Y. Yao. Phys. Rev. Lett., 107:076802, 2011.

[11] M. Ezawa. New Journal of Physics, 14:033003, 2012.

[12] P. Vogt, P. De Padova, C. Quaresima, E. Frantzeskakis, M.C. Asensio, A. Resta, B. Ealet, and G. Le Lay. Phys. Rev. Lett., 108:155501, 2012.

[13] J. Gao and J. Zhao. Sci. Rep., 2:861, 2012.

[14] A. Fleurence, R. Friedleiner, T. Ozaki, H. Kawai, Y. Wang, and Y. YamadaTakamura. Phys. Rev. Lett., 108:245501, 2012.

[15] L. Meng, Y. Wang, L. Zhang, S. Du, R. Wu, L. Li, Y. Zhang, G. Li, H. Zhou, W.A. Hofer, and H.-J. Gao. Nano Lett., 13:685-690, 2013.

[16] D. Chiappe, E. Scalise, E. Cinquanta, C. Grazianetti, B. van der Broek, M. Fanciulli, M. Houssa, and A. Molle. Adv. Mater., 26:2096-2101, 2014. 
[17] A. Molle, A. Lamperti, D. Rotta, Fanciulli M., E. Cinquanta, and C. Grazianetti. Adv. Mater., 26:2096-2101, 2014.

[18] M. De Crescenzi, I. Berbezier, M. Scarselli, P. Castrucci, M. Abbarchi, A. Ronda, F. Jardali, J. Park, and H. Vach. ACS Nano, 10:11163-11171, 2016.

[19] C. Volders, E. Monazami, G. Ramalingam, and P. Reinke. Nano Lett., 17:1161-1166, 2017.

[20] Q. Yao, R. van Bremen, and H.J.W. Zandvliet. Appl. Phys. Lett., 109:243105, 2016.

[21] C. Volders, E. Monazami, G. Ramalingam, and P. Reinke. Nano Lett., 17:299307, 2017.

[22] Y. Feng, D. Liu, B. Feng, X. Liu, L. Zhao, Z. Xie, Y. Liu, A. Liang, C. Hu, Y. Hu, S. He, G. Liu, J. Zhang, C. Chen, Z. Xu, L. Chen, K. Wu, Y.-T. Liu, H. Lin, Z.-Q. Huang, C.-H. Hsu, F.-C. Chuang, A. Bansil, and X.J. Zhou. PNAS, 113:51, 2016.

[23] S.K. Mahatha, P. Moras, V. Bellini, P.M. Sheverdyaeva, C. Struzzi, L. Petaccia, and C. Carbone. Phys. Rev. B., 89(201416(R)), 2014.

[24] P. De Padova, P. Vogt, A. Resta, J. Avila, I. Razado-Colambo, C. Quaresima, C. Ottaviani, B. Olivieri, T. Bruhn, T. Hirahara, T. Shirai, S. Hasegawa, M.C. Asensio, and G. Le Lay. Appl. Phys. Lett., 102:163106, 2013.

[25] D. Tsoutsou, E. Xenogiannopoulou, E. Golias, P. Tsipas, and A. Dimoulas. Appl. Phys. Lett., 103:231604, 2013.

[26] R. Quhe, Y. Yuan, J. Zheng, Y. Wang, Z. Ni, J. Shi, D. Yu, J. Yang, and J. Lu. Sci. Rep., 4:5476, 2014.

[27] P.M. Sheverdyaeva, S.K. Mahatha, P. Moras, L. Petaccia, G. Fratesi, G. Onida, and C. Carbone. ACS Nano, 11:975-982, 2017.

[28] B. Feng, Z. Ding, S. Meng, Y. Yao, X. He, P. Cheng, L. Chen, and K. Wu. Nano Lett., 12:3507-3511, 2012.

[29] C.-L. Lin, R. Arafune, K. Kawahara, N. Tsukahara, E. Minamitani, Y. Kim, N. Takagi, and M. Kawai. Appl. Phys. Express, 5:045802, 2012.

[30] H. Jamgotchian, Y. Colignon, N. Hamzaoui, B. Ealet, J.-Y. Hoarau, B. Aufray, and J.P. Bibérian. J. Phys. Condens. Matter, 24:172001, 2012.

[31] D. Chiappe, C. Grazianetti, G. Tallarida, M. Fanciulli, and A. Molle. Adv. Mater., 24:5088-5093, 2012.

[32] W. Wang, W. Olovsson, and R.I.G. Uhrberg. Phys. Rev. B., 92:205427, 2015. 
[33] R. Arafune, C.-L. Lin, K. Kawahara, N. Tsukahara, E. Minamitani, Y. Kim, N. Takagi, and M. Kawai. Surf. Sci., 608:297-300, 2013.

[34] H. Enriquez, S. Vizzini, A. Kara, B. Lalmi, and H. Oughaddou. J. Phys. Condens. Matter, 24:314211, 2012.

[35] J. Zhao, H. Liu, Z. Yu, R. Quhe, S. Zhou, Y. Wang, C.C. Liu, H. Zhong, N. Han, J. Lu, Y. Yao, and K. Wu. Progres in Materials Science, 83:24-151, 2016.

[36] F. Liu, C.-C. Liu, K. Wu, F. Yang, and Y. Yao. Phys. Rev. Lett., 111:066804, 2013.

[37] P. De Padova, J. Avila, A. Resta, I. Razado-Colambo, C. Quaresima, C. Ottaviani, B. Olivieri, T. Bruhn, P. Vogt, and M.C. Asensio. J. Phys. Condens. Matter, 25:382202, 2013.

[38] J. Chen, Y. Du, Z. Li, W. Li, B. Feng, J. Qiu, P. Cheng, S.H. Dou, L. Chen, and K. Wu. Sci. Rep., 5:13590, 2015.

[39] T. Shirai, T. Shirasawa, T. Hirahara, N. Fukui, T. Takahashi, and S. Hasegawa. Phys. Rev. B., 89:241403(R), 2014.

[40] E. Bauer. Reg. Prog. Pys., 57:895-906, 1994.

[41] P. Vogt, P. Capiod, M. Berthe, A. Resta, P. De Padova, T. Bruhn, G. Le Lay, and B. Grandidier. Appl. Phys. Lett., 104:021602, 2014.

[42] B. Feng, H. Li, C.-C. Liu, T.-N. Shao, P. Cheng, Y. Yugui, S. Meng, L. Chen, and K. Wu. ACS Nano, 7, 2013.

[43] M. Giessen. Progress in Surface Science, 68:1-153, 2001.

[44] J. de la Figuera, N.C. Bartelt, and K.F. McCarty. Surf. Sci., 600(4062), 2006.

[45] D. Schwarz, R. van Gastel, H.J.W. Zandvliet, and B. Poelsema. Phys. Rev. Lett., 109(016101), 2012.

[46] D. Schwarz, R. van Gastel, H.J.W. Zandvliet, and B. Poelsema. Phys. Rev. B., 85(235419), 2012. 



\section{Chapter 4}

\section{Germanene: a germanium analogue of graphene}

\subsection{Introduction}

The discovery of graphene, the first two-dimensional material, has led to a cornucopia of new and exciting physics [?]. Graphene consists of $\mathrm{sp}^{2}$ hybridized carbon atoms that are arranged in a planar configuration. The observation that a single free-standing sheet of atoms is stable was already quite a surprise, since the Mermin and Wagner theorem [?] states that a two-dimensional crystal cannot exhibit long-range order at any finite temperature. In 1987 Nelson and Peliti [?] performed a theoretical study on the intricate interplay between crystalline order and thermal fluctuations in crystalline membranes. They showed that the anharmonic coupling between in-plane and out-of-plane (flexural) lattice vibrations is of crucial importance for the stability of a membrane, without this anharmonic coupling the membrane would be fully crumpled. As a result of this anharmonic coupling, the membrane becomes overall more or less flat, but the membrane displays strong intrinsic corrugations (ripples) that are characterized by a power-law behaviour of the atomic-displacement correlations functions. The system remains approximately two-dimensional (with typical out-of-plane displacements that are much smaller than the sample size) and approximately crystalline (with a crystalline order which is preserved at finite, but very large distances) [?,?,?]. At least for rigid systems, such as graphene, this means that one can safely use the term "two-dimensional crystal" for any practical purpose [?]. Experimental studies have revealed that freely suspended graphene is indeed rippled [?].

The impressive rise of graphene has spurred many scientists to look for alter- 
native two-dimensional materials. The exploration of this new realm of twodimensional materials has barely begun, its promises have not yet fully materialized, and the extent of its potential for new physics and devices remains largely unexploited. The most obvious alternatives for graphene are the group-IV elements, i.e. silicon and germanium. The electron configurations of germanium, silicon and carbon are very similar since all three elements have four electrons in their outermost $\mathrm{s}$ and $\mathrm{p}$ shells. The energetically most favourable crystal structure of silicon and germanium is the diamond structure [?]. The diamond lattice consists of two interpenetrating fcc sub-lattices and each atom of these fcc sub-lattices is surrounded by four neighbours. The covalent bonds between the atoms are all equivalent and have a hybridized $\mathrm{s}, \mathrm{p}_{x}, \mathrm{p}_{y}, \mathrm{p}_{z}$ character $\left(\mathrm{sp}^{3}\right)$. For carbon, another allotrope is found in nature that consists of a stack of sheets with a honeycomb structure (graphene). This carbon allotrope is named graphite and is under normal conditions, i.e. room temperature and atmospheric pressure, thermodynamically more stable than the carbon allotrope that has the diamond structure [?]. The three in-plane covalent bonds of graphene make angles of $120^{\circ}$ with each other and have a hybridized $2 \mathrm{~s}, 2 \mathrm{p}_{x}$ and $2 \mathrm{p}_{y}$ character $\left(\mathrm{sp}^{2}\right)$, whereas the remaining orbital has a pure $2 \mathrm{p}_{z}$ character and points in a direction normal to the graphene sheet, and thus responsible for the metallic character of the system. The $2 \mathrm{p}_{z}$ electrons are itinerant and distributed throughout the whole carbon sheet. These $2 \mathrm{p}_{z}$ orbitals give rise to the formation of the $\pi$-bonding and $\pi^{*}$-antibonding orbitals, which are largely responsible for the van der Waals interaction between the graphene sheets in graphite. For silicon and germanium such graphite-like allotropes have not been found in nature and therefore the silicon and germanium 'graphite' allotropes, hereafter referred as silicene and germanene respectively, are appealing candidates for synthesis.

Germanene, silicene and graphene share several very peculiar and interesting electronic properties. The electrons near the $\mathrm{K}$ and $\mathrm{K}^{\prime}$ points of the Brillouin zone behave as relativistic massless particles. The electronic states of graphene near the Dirac points are described by a linear dispersion relation with a Fermi velocity of about $10^{6} \mathrm{~m} / \mathrm{s}$. Already in the first experimental studies charge carrier mobilities as high as $15.000 \mathrm{~cm}^{2} / \mathrm{Vs}$ have been obtained [?]. Another hallmark of these two-dimensional Dirac materials is that they display an anomalous ('halfinteger') quantum Hall effect, which we will briefly touch upon in Sec. 4.4. There are, however, also a few differences between germanene and silicence on the one hand and graphene on the other hand. Firstly, the honeycomb lattice of graphene is fully planar, whereas the honeycomb lattices of germanene and silicene are predicted to be buckled [?,?, ?] (see Fig. 4.1). Secondly, due to the larger atomic number of germanium and silicon as compared to carbon, these materials have a much stronger spin-orbit coupling. It should be noted here that the already very weak spin-orbit coupling in graphene is even further suppressed by its flatness. A small buckling will increase the spin-orbit coupling by orders of magnitude [?]. 
The spin-orbit coupling results in the opening of a small band gap at the Dirac points in the interior of the material, topological protected gapless helical modes at the edges of the two-dimensional material and a quantum spin Hall effect which is characterized by spin current transport via the edges modes [?,?]. The spin-orbit gap in graphene, silicene and germanene are $<0.05 \mathrm{meV}, 1.55 \mathrm{meV}$ and $23.9 \mathrm{meV}$, respectively [?,?,?,?,?]. This means that the quantum spin Hall state is only experimentally accessible for silicene and germanene [?,?].

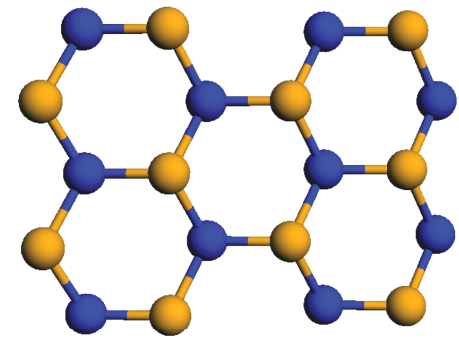

(a)

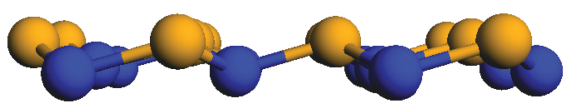

(b)

Figure 4.1 - Ball and stick model of germanene. The honeycomb lattice is composed of two triangular sub-lattices. Left panel: top view. Right panel: side view.

The first reports on the synthesis of silicene date back to 2010 [?, ?, ?, ?, ?, ?], followed by germanene in 2014 [?,?,?,?]. Meanwhile silicene has been grown on several substrates (Ag(110), $\mathrm{Ag}(111), \mathrm{ZrB}_{2}(0001)$ and $\left.\operatorname{Ir}(111)\right)$ and characterized by a variety of surface science techniques $[?, ?, ?, ?, ?, ?, ?]$. This chapter focuses on the properties of germanene.

It is important to point out here that there are, besides graphene, silicene and germanene, a few more two-dimensional Dirac materials, such as stanene (Sn), $d$-wave superconductors (YBCO, LSCO), and transition metal-oxides as $\left(\mathrm{VO}_{2}\right)_{n} /\left(\mathrm{TiO}_{2}\right)$ [?]. In the last few years we have seen a flood of articles on other types of two-dimensional materials such as phosphorene, arsenene, transition metal(di)chalcogenides (i.e. $\mathrm{MoS}_{2}$ ), organic crystals and artificial twodimensional lattices [?].

The chapter starts with an update on the current status of germanene. We will start with a brief theoretical section where also density functional theory calculations of free-standing single-layer and bilayer germanene are presented. In Sec. 4.3 the various methods to synthesize germanene will be presented and discussed. Subsequently we will present a section that discusses the peculiar electronic properties of germanene. We will elaborate on how the quantum spin Hall effect can be measured and how a substantial band gap can be opened in germanene. The chapter ends with a short outlook. 


\subsection{Theoretical calculations}

The first quantum mechanical ab initio calculations on 'graphite-like' silicon and germanium sheets were performed by Takeda and Shiraishi [?]. They found that the configuration with the lowest energy is buckled, i.e. the two sub-lattices of the honeycomb lattice are slightly displaced with respect to each other in a direction normal to the sheet. The $a b$ initio calculations of Takeda and Shiraishi also revealed that silicene and germanene are semi-metals. Although these authors did not explicitly discuss the k-dependence of the electronic states that are responsible for the semi metallic character, it is clear from their energy band structure calculations that the dispersion relations are linear in $\mathrm{k}$. In a later tight binding calculation Guzmán-Verri and Lew Yan Voon [?] pointed out that silicene has Dirac cones at the $\mathrm{K}$ and $\mathrm{K}^{\prime}$ points of the Brillouin zone. These Dirac cones are robust against the buckling of the silicene lattice and therefore free-standing silicene is a two-dimensional Dirac fermion system. A few years later Cahangirov et al. [?] arrived at a similar conclusion for germanene and also demonstrated its structural stability with respect to atomic vibrations.

Since the theoretical prediction of stable free-standing germanene [?], its electronic and structural properties have been extensively studied by means of density functional theory (DFT) calculations [?,?,?,?,?,?,?,?]. The calculations that are presented here are performed using the projected augmented wave (PAW) method [?] within the generalized gradient approximation (GGA) [?] as implemented in the VASP package [?,?]. In comparison to graphene, the $\pi$-bonding in germanene is significantly weaker [?]. Apart from the increased interatomic distance, this results in a smaller energy splitting between the bonding and antibonding orbitals having significant consequences for the structure of germanene. Specifically, as can be deduced from the band structure of planar germanene (Fig. 4.2(a)), the low lying antibonding bands are partially occupied in the vicinity of the $\Gamma$-point, resulting in a finite density of states at the Fermi level. Being unfavourable from an energetic point of view, such states tend to be unoccupied, which is achieved in free-standing germanene by forming a buckled structure (Fig. 4.2(c)) at the expense of lowering the point group symmetry from $D_{6 h}$ to $D_{3 d}$ [?]. The buckling $\Delta$, that is the vertical separation between the two sub-lattices, is ultimately determined by a balance between the electronic and elastic energies. For free-standing germanene, DFT studies report $\Delta$ in the range of 0.64 to $0.74 \AA$, depending on the exact computational scheme [?]. Although configurations with a considerably larger buckling $(>2 \AA)$ also appear at the total energy landscape [?,?], they do not seem to be realistic in view of the presence of imaginary modes in the phonon spectrum, implying dynamical instability of the corresponding structures. The buckling of germanene plays a crucial role in the formation of its intrinsic electronic properties. Particularly, the non-planar 
geometry along with a strong spin-orbit interaction in germanene facilitates the opening of a considerable band gap at the Dirac point of the order of the thermal energy at room temperature $(\approx 24 \mathrm{meV})[?, ?]$.

The structural and electronic properties of germanene can be significantly modified by interactions with the underlying substrate [?,?]. One mechanism proven to be relevant for the magnitude of the buckling is lateral strain [?,?], appearing for deposited structures due to improper matching between the lattice constants of the substrate and adsorbed layer. Similar to graphene, sub-lattice symmetry in supported germanene is generally not preserved, resulting in the opening of a band gap [?,?].
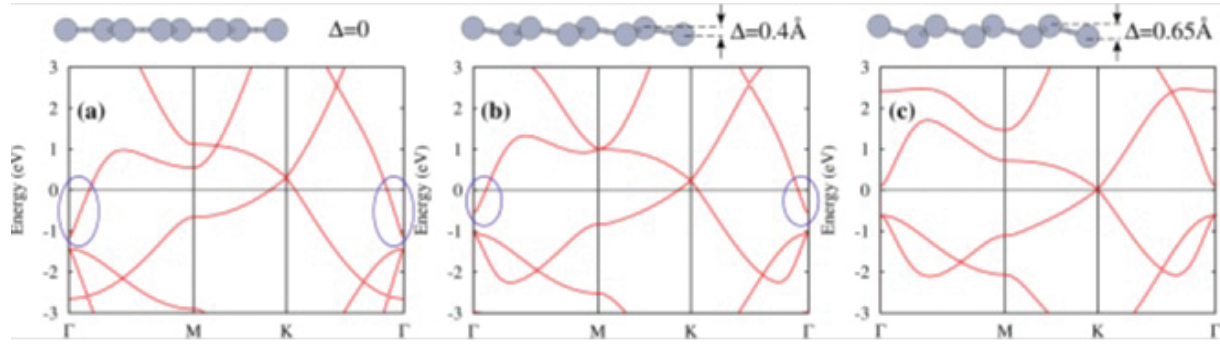

Figure 4.2 - Electronic band structure of germanene calculated within the DFT for different values of the buckling $\Delta$. Zero energy corresponds to the Fermi energy. Blue circles denote the antibonding band crossing the Fermi energy at low buckling values.

Group-IV element analogues of few-layer graphene, bilayer graphene in particular, have been studied theoretically and experimentally. Specifically bilayer silicene has been explored in quite some detail. DFT calculations of free-standing bilayer silicene predict several (meta)stable structures, where the two silicene layers are either in an $\mathrm{AA}$ or $\mathrm{AB}$ stacking [?,?,?]. Calculations of free-standing bilayer germanene give two similar, locally stable, optimized structures, with an AA-stacked structure (see Fig. 4.3) that is $\approx 23 \mathrm{meV} /$ atom more stable than an AB-stacked structure (see Fig. 4.4). The two Ge layers in the AA structure are planar, whereas in the $A B$ structure they are buckled, reflecting an $\mathrm{sp}^{2}$ - and $\mathrm{sp}^{3}$ type bonding in the $\mathrm{AA}$ and $\mathrm{AB}$ structures, respectively. The $\mathrm{sp}^{2}$ bonding in the AA structure does not give rise to a strong intra-layer $\pi$-bonding like in bilayer graphene, however. In bilayer graphene the intra-layer bonding is strong, and the interlayer bonding originates from a weak, van der Waals interaction. In contrast, in AA bilayer germanene the intra- and interlayer interactions are of comparable strength. This is reflected in the structure, where both the intra- and the interlayer Ge-Ge bonds have a bond length of $2.56 \AA$. In the $A B$ structure the intra- and interlayer Ge-Ge bonds have bond lengths of $2.49 \AA$, respectively 2.68 $\AA$, which indicates that also in this structure intra- and interlayer interactions are comparable. 

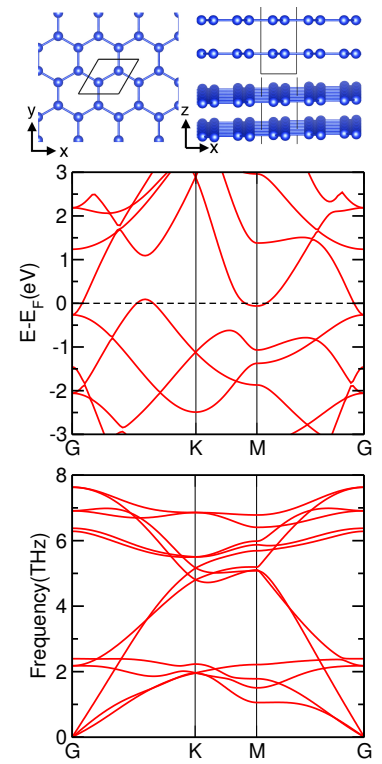

Figure 4.3 - Top: top and side view of optimized AA-stacked structure of bilayer germanene (DFT calculation using the PBE/GGA functional); the optimized in-plane lattice constant is $4.43 \AA$. Middle: the electronic bands along specific high symmetry directions in the two-dimensional Brillouin zone; the zero of energy is at the Fermi level. Bottom: the phonon dispersions along the same directions.
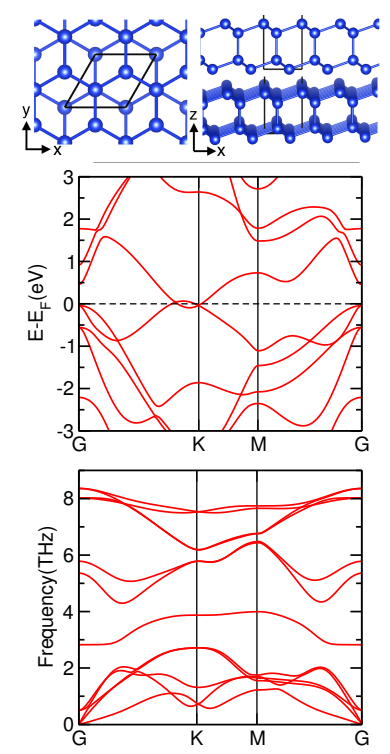

Figure 4.4 - As Fig. 4.3 for the optimized AB-stacked structure of bilayer germanene; the optimized in-plane lattice constant is $4.08 \AA$.

The strong interlayer bonding in bilayer germanene yields an electronic structure that is very different from that of bilayer graphene. In the bilayer germanene 
AA structure band minima at $\Gamma$ and at $\mathrm{M}$ can be found close to the Fermi level, whereas the band maxima are located at $\Gamma$ and $\sim 0.6 \Gamma \mathrm{K}$, respectively (see Fig. 4.3). The AB structure shows band minima at $\sim 0.9 \Gamma \mathrm{K}$, and band maxima at $\Gamma$ and $\sim 0.9 \Gamma \mathrm{K}$ close to the Fermi level, respectively (see Fig. 4.4). DFT calculations with a conventional functional (PBE) based upon the generalized gradient approximation (GGA) [?], yield a semimetal for both structures. It is conceivable that a gap will be opened if a more advanced approach, such as GW [?,?], is used to calculate the spectrum. The local stability of both the AA and AB structures is demonstrated by the corresponding phonon spectra, in particular by the absence of any modes with an imaginary frequency, see Figs. 3 and 4 . In bi- and multilayer graphene the weak interlayer bonding gives rise to low frequency optical modes [?]. The absence of any such modes in bilayer germanene is consistent with a strong interlayer bonding.

Whether adsorption of germanene or bilayer graphene on a substrate preserves the free-standing structures obviously depends on the interaction with the substrate. Adsorption of graphene, or its insulating counterpart, hexagonal boron nitride (h-BN), on metal surfaces ranges from weak physisorption to strong chemisorption, depending on the substrate [?,?,?,?,?,?]. The perturbation of the (electronic) structure of graphene then increases with increasing graphenesubstrate interaction. Silicene is generally more reactive than graphene. The substrate-adsorbent interaction in silicene on $\mathrm{Ag}(111)$, for instance, is considerable, and although the adsorption preserves the hexagonal structure of silicene, the Ag substrate induces a sizable out-of-plane buckling of the Si atoms [?]. Hybridization between the silicene and the Ag states then leads to a large perturbation of the silicene electronic structure. Given the similarities between silicene and germanene, it is reasonable to assume that germanene behaves more like silicene than like graphene, when adsorbed on a metal substrate. Various structural models have been proposed to describe the existing experiments on germanene [?,?,?,?], see the next section. Their reliability has however not been unequivocally established. It is, for instance, worth noting that the honeycomb structures observed in the STM experiments on Au-Ge and Pt-Ge [?, ?, ?] can also be attributed to the hexagonal (111) surfaces of $\mathrm{Ge}_{2} \mathrm{Pt}$ or $\mathrm{Ge}_{2} \mathrm{Au}$ fcc crystals, where the honeycomb termination only might be visible in experiments due to the unequal electronic density in the vicinity of $\mathrm{Ge}$ and $\mathrm{Pt}(\mathrm{Au})$ atoms and also due to the symmetry-governed vertical displacement of surface atoms. 


\subsection{Synthesis of germanene}

\subsubsection{Structural properties on various substrates}

The synthesis of germanene and germanene-related materials was initiated by a report by Bianco et al. [?] on the preparation and exfoliation of germanane $(\mathrm{GeH})$. Germanane, i.e. hydrogen terminated germanene, was successfully prepared via the topochemical deintercalation of $\mathrm{CaGe}_{2}$. Germanane sheets can be obtained by simple exfoliation of the layered van der Waals solid. At ambient conditions germanane is very stable and only oxidizes in a time span of several months. This stability is an important prerequisite for the usage of germanane in any technological application. The strong potential of germanane for technological applications is fueled by theoretical calculations, which predict a direct band gap of $1.5 \mathrm{eV}$ and an electron mobility that is substantially higher than that of bulk germanium [?,?].

As pointed out in the preceding section free-standing germanene is stable against local lattice distortions. To date germanene has been reported to be synthesized on only a few substrates. In July $2014 \mathrm{Li}$ et al. [?] reported the growth of germanene on $\mathrm{Pt}(111)$. Germanium was deposited on a pristine $\mathrm{Pt}(111)$ substrate at room temperature under ultra-high vacuum conditions from a germanium rod mounted in an electron-beam evaporator. After deposition the Pt substrate was annealed at a temperature in the range of $600-750 \mathrm{~K}$ for $30 \mathrm{~min}$. Using low energy electron diffraction and scanning tunneling microscopy they found a ( $\sqrt{19} \times$ $\sqrt{19})$ periodicity with respect to the $\mathrm{Pt}(111)$ substrate. Their scanning tunneling microscopy data revealed the presence of a continuous and well-ordered $(\sqrt{19} \times$ $\sqrt{19})$ superstructure, which they interpreted as a germanene adlayer on the $\mathrm{Pt}(111)$ substrate. Unfortunately, Li et al. [?] did not manage to obtain atomic resolution. Line scans recorded with a scanning tunneling microscope showed that the $(\sqrt{19} \times \sqrt{19})$ superstructure has a corrugation of about $0.6 \AA$. The authors ascribed this corrugation to Ge atoms that are located at different positions on the $\operatorname{Pt}(111)$ substrate. A density functional theory calculation revealed that the $(\sqrt{19} \times \sqrt{19})$ superstructure coincides with a germanene layer that has a $(3 \times 3)$ periodicity (see Fig. 4.5(A)). Recently, Ŝvec et al. [?] suggested that the $(\sqrt{19} \times \sqrt{19})$ reconstruction on $\mathrm{Pt}(111)$ is actually not germanene, but a surface alloy composed of $\mathrm{Ge}_{3} \mathrm{Pt}$ tetramers that resembles a twisted kagome lattice. These authors based their conclusion on a comprehensive study of the closely related $\mathrm{Si} / \mathrm{Pt}(111)$ system.

The second paper on the synthesis of germanene is by Davila et al. [?]. They reported the growth of germanene on $\mathrm{Au}(111)$. These authors performed a combined scanning tunneling microscopy and low energy electron diffraction study and they identified three different phases: a $(\sqrt{7} \times \sqrt{7}) R 19.1^{\circ}$ phase, a 


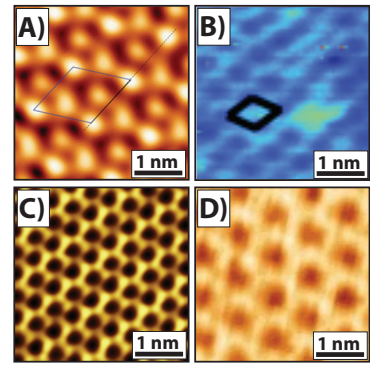

Figure 4.5 - STM images of germanene sheets grown by several research groups. For comparison all the images have the same size of $4 \mathrm{~nm} \times 4 \mathrm{~nm}$. (A) STM image of the germanene $(\sqrt{19} \times \sqrt{19})$ superstructure on $\operatorname{Pt}(111)$. $(\mathrm{V}=1 \mathrm{~V}$ and $\mathrm{I}=0.05 \mathrm{nA})$, [?] (B) STM image of the germanene $(\sqrt{3} \times \sqrt{3})$ superstructure on $\mathrm{Au}(111)(\mathrm{V}=1.12 \mathrm{~V}$ and $\mathrm{I}=1.58 \mathrm{nA}$; the $\mathrm{Au}(111)(\sqrt{7} \times$ $\sqrt{7}$ ) unit cell is outlined in black), [?] (C) STM image of the germanene honeycomb layer on a $\mathrm{Ge}_{2} \mathrm{Pt}$ cluster $(\mathrm{V}=0.5 \mathrm{~V}$ and $\mathrm{I}=0.2 \mathrm{nA})$, [?] and (D) STM image of the germanene $(3 \times 3)$ superstructure on $\mathrm{Al}(111)(\mathrm{V}=0.7 \mathrm{~V}$ and $\mathrm{I}=0.3 \mathrm{nA})$, [?]. Printed with permission. Image courtesy of Wiley [?], Institute of Physics [?,?] and the American Chemical Society [?].

$(5 \times 5)$ phase and a $(\sqrt{19} \times \sqrt{19}) R 23.4^{\circ}$ phase (all referred to the periodicity of the $\mathrm{Au}(111)$ substrate). The $(\sqrt{7} \times \sqrt{7})$ phase (also referred as the $(\sqrt{3} \times \sqrt{3})$ phase) exhibits a nearly flat honeycomb structure. Based on these observations Davila et al. ascribed the $(\sqrt{7} \times \sqrt{7})$ phase to germanene (see Fig. 4.5(B)). Their conclusion is supported by synchroton radiation core-level spectroscopy measurements and density functional theory calculations.

A few days after the appearance of Davila's publication another paper appeared by Bampoulis et al. [?], where the formation of germanene terminated $\mathrm{Ge}_{2} \mathrm{Pt}$ clusters was reported. Bampoulis et al. deposited a few monolayers Pt on a $\mathrm{Ge}(110)$ substrate and subsequently annealed the sample at $1100 \mathrm{~K}$. The bulk phase diagram of Pt-Ge system exhibits an eutectic at $1043 \mathrm{~K}$. This occurs at a composition of $22 \%$ and $78 \% \mathrm{Pt}$ and $\mathrm{Ge}$, respectively. Low energy electron microscopy (LEEM) images revealed that slightly above this eutectic temperature liquid drops are formed and move as large entities across the surface [?]. Interestingly, for the other low-index surfaces of germanium an eutectic top-layer is formed, rather than droplets [?,?,?,?]. This difference is due to the relatively high surface free energy of $\mathrm{Ge}(110)$ as compared to the $\mathrm{Ge}(001)$ and $\mathrm{Ge}(111)$ surfaces [?,?]. Upon cooling down the eutectic $\mathrm{Pt}_{0.22} \mathrm{Ge}_{0.78}$ droplets that have been formed on the Ge(110) surface they solidify and spinodal decomposition occurs. The eutectic phase separates in a pure Ge phase and $\mathrm{Ge}_{2} \mathrm{Pt}$ phase, respectively. The phase with the lowest surface free energy, i.e. Ge, segregates towards to the surface of the droplet, whereas the interior is composed of the other phase, i.e. the $\mathrm{Ge}_{2} \mathrm{Pt}$ alloy. After solidification the surface of the droplets exhibit a buckled honeycomb structure. Scanning tunneling microscopy images reveal and atom- 
ically resolved buckled honeycomb lattice with a nearest-neighbor distance of $2.5 \pm 0.1 \AA$ (see Fig. 4.5(C). Bampoulis et al. [?] also found that the honeycomb lattice is buckled and composed of two triangular sub-lattices, which are displaced with respect to each other by only $0.2 \AA$. This buckling is much smaller than the $0.65 \AA$ that is reported in several density functional theory calculations for free-standing germanene. As has been shown above free-standing germanene is metallic for buckling values smaller than about $0.6 \AA$. The scanning tunneling spectra reported by Bampoulis et al. [?] revealed a metallic-like behavior. Two remarks are in place here (1) it is very well possible that the electronic states of the germanene top layer hybridizes with the underlying substrate and (2) the recorded scanning tunneling spectra are also affected by the electronic structure of the scanning tunneling microscope tip.

Although the experimental studies have revealed honeycomb-like reconstructions [?, ?, ?, ?] it remains to be seen if the grown germanene indeed behaves as a 2D Dirac fermion system. Angle-resolved photoemission experiments are needed to reveal if the synthesized germanene sheets indeed have Dirac cones at the K points of the surface Brillouin zone. Another test would be to apply a magnetic field normal to the germanene sheet and measure the Landau levels with for instance scanning tunneling microscopy [?]. The presence and separation of the Landau levels will immediately reveal whether one deals with a two-dimensional electron gas or a two-dimensional Dirac fermion system.

To date germanene has only been grown on metallic substrates. It is very likely that the relevant electronic states of germanene near the Fermi level hybridize with electronic states of the metallic substrate and destroy the two-dimensional Dirac character of the germanene. It would be a huge step forward if germanene could be synthesized on a wide band gap material. A possible candidate would be hexagonal boron nitride (h-BN). h-BN has a band gap of about $6 \mathrm{eV}$ and its lattice constant $(2.5 \AA)$ is almost identical to the nearest neighbor distance of germanene (see Fig. 4.6).

\subsubsection{Electronic properties: Scanning Tunneling Spectroscopy study}

Recently, Zhang et al. [?] performed a room temperature scanning tunneling spectroscopy study of germanene grown on $\mathrm{Ge}_{2} \mathrm{Pt}$. They showed that the density of states of the interior of the germanene layer exhibits a well-defined V-shape, which is a hallmark of a two-dimensional Dirac material. Here we extend this scanning tunneling spectroscopy study to cryogenic temperatures. After correcting the data for thermal broadening a virtually perfect $\mathrm{V}$-shaped density of states is found. Inspired by these results we have attempted to directly measure 


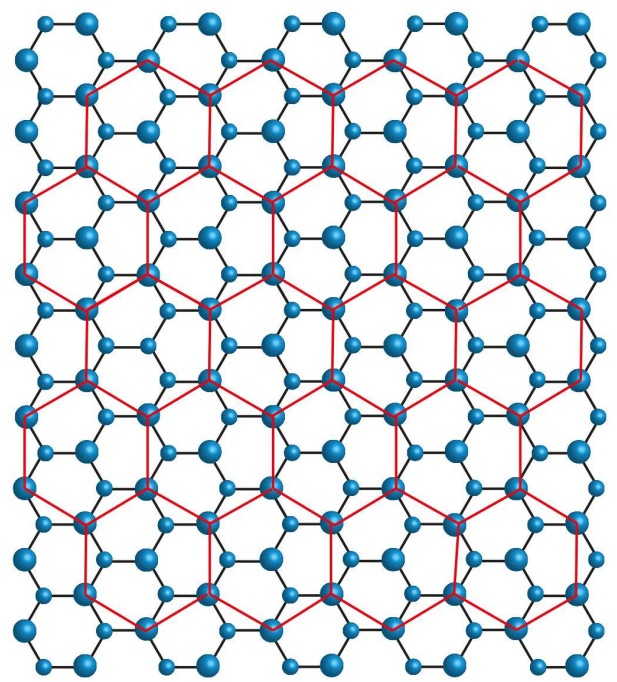

Figure 4.6 - Germanene sheet (red honeycomb) on h-BN substrate (blue honeycomb). The nearest-neighbour distance of germanene and the lattice constant of h-BN are both $2.5 \AA$.

the energy dispersion relation, $E(k)$, by recording spatial maps of the differential conductivity near the edges of the germanene. However, we did not find any sign of Friedel oscillations, which suggests that the electrons are not scattered at the edges of the germanene. The absence of these Friedel oscillations is believed to be due to a suppressed backscattering as a result of Klein tunneling [?,?].

Experiments were performed with two separate ultra-high vacuum scanning tunneling microscopes (STM), a room temperature STM (Omicron STM-1) and a low-temperature STM (Omicron LT-STM). The base pressure in both ultra-high vacuum systems is below $3 \times 10^{-11}$ mbar.

Samples were prepared in the same manner as described in ref. [?]. Ge(110) samples were cut from flat $10 \times 10 \times 0.4 \mathrm{~mm}$, single-side-polished n-type substrates. The samples were subsequently mounted on Mo holders and introduced into the vacuum systems. Subsequently we deposited $\sim 3$ monolayers of $\mathrm{Pt}$ at room temperature through resistive heating of a high purity $\mathrm{Pt}(99.995 \%)$ wire wrapped around a $\mathrm{W}$ filament. In order to obtain the germanene terminated $\mathrm{Ge}_{2} \mathrm{Pt}$ clusters, the samples were flash annealed $(T=1100 \pm 25 \mathrm{~K})$, and then slowly cooled to room temperature before placing them into the microscope. The sample in the LT-STM was cooled to $77 \mathrm{~K}$ before observation.

Differential conductivity $(d I / d V)$ measurements were acquired by numerical differentiation of $I(V)$ curves, which were obtained through averaging a $30 \times 30$, $1 \mathrm{~nm}$ spaced grid of individual $I(V)$ spectra. The $I(V)$ spectra were recorded with the feedback loop disabled. In order to determine the quality and reliability of the $d I / d V$ spectra obtained by numerical differentiation we have performed sev- 
eral test experiments where we simultaneously recorded the $d I / d V$ signal using a lock-in technique while ramping the voltage. Since the differences between the $d I / d V$ signals obtained by both methods are very small we have decided to present the $d I / d V$ curves obtained via numerical differentiation.

An atomic force microscopy image of a typical $\mathrm{Ge}_{2} \mathrm{Pt}$ cluster is shown in Fig. 4.7. The $\mathrm{Ge}_{2} \mathrm{Pt}$ clusters are similar to the ones observed previously in refs. [?] and [?] and are either flat-topped or pyramidal shaped. The footprint of the clusters varies from elongated to near-circular, with typical lateral sizes ranging from a few hundred $\mathrm{nm}$, up to $\sim 1.5 \mu \mathrm{m}$ and heights up to $\sim 100 \mathrm{~nm}$. The flat-topped clusters have a buckled honeycomb top layer, with a lattice constant of $0.25 \pm 0.01 \mathrm{~nm}$, which is very close to that of free-standing germanene (see inset of Fig. 4.7 for a small scale scanning tunneling microscopy image). For more detailed information regarding the properties of the clusters, the buckled honeycomb lattice and the bare Ge(110) surface, we refer to ref. [?]. Owing to their small size, the germanene coated $\mathrm{Ge}_{2} \mathrm{Pt}$ clusters are mainly of fundamental interest.

Figure 4.8 shows typical differential conductivity $(d I / d V)$ spectra of a flat-topped cluster, recorded at $293 \mathrm{~K}$ and $77 \mathrm{~K}$, respectively. The $I(V)$ curves are shown in the insets of Fig. 4.8. In the vicinity of the Fermi level, the differential conductivity is proportional to the density of states (DOS). The V-shaped density of states, which is a hallmark of a 2D Dirac system, is clearly present for both temperatures. However, we do note some discrepancies compared to the density of states for this system and an ideal 2D spectrum as well as some small differences between the two measurements. Firstly, the measurements are not fully symmetrical around the Dirac point, as would be expected for a 2D Dirac system due to the intrinsic electron-hole symmetry. Secondly, the asymmetry differs for the two measurements. One has to realize that also the electronic structure of the

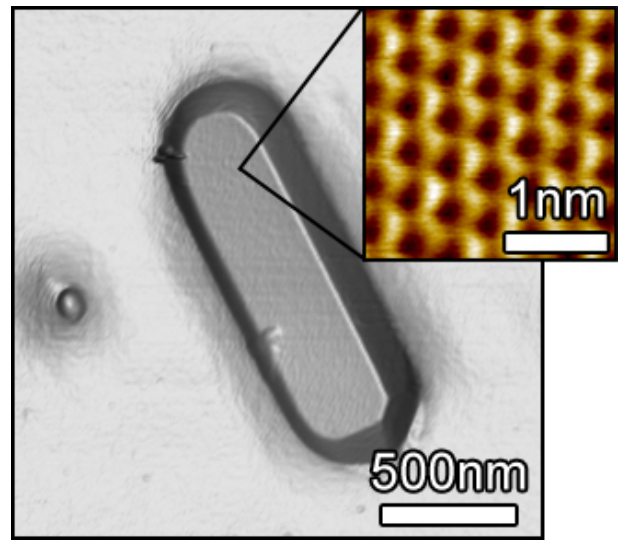

Figure 4.7 - Atomic force microscopy image of a flat-topped $\mathrm{Ge}_{2} \mathrm{Pt}$ cluster. Inset shows an STM image of the buckled honeycomb structure on top of these $\mathrm{Ge}_{2} \mathrm{Pt}$ clusters. See ref. [?] for more details. 
scanning tunneling microscope tip and the underlying metallic $\mathrm{Ge}_{2} \mathrm{Pt}$ substrate have an effect on the scanning tunneling spectra. For a comparison to the closely related silicene system the interested reader is referred to refs. [?, ?, ?, ?]. The scanning tunneling spectroscopy measurements were performed with separate STMs, and thus also with different scanning tunneling microscopy tips. Any asymmetry of the density of states of the scanning tunneling microscopy or metallic $\mathrm{Ge}_{2} \mathrm{Pt}$ substrate in the vicinity of the Fermi level will also affect the scanning tunneling spectra. However, we would like to emphasize that even from cluster to cluster we found, using the same tip, small differences in: (1) the asymmetry of the spectra, (2) the vertical offset at zero bias, i.e. the metallicity and (3) the exact position of the Dirac point. We ascribe these small differences to local variations in the doping and hybridization/coupling of the germanene layer to the underlying $\mathrm{Ge}_{2} \mathrm{Pt}$ substrate. In addition, we have repeated our experiments with several STM tips and samples and we found that in all cases the $d I / d V$ spectra recorded on top of the flat-topped clusters exhibit a V-shape.

For an ideal, i.e. symmetrical and semimetallic, 2D Dirac system, the typically

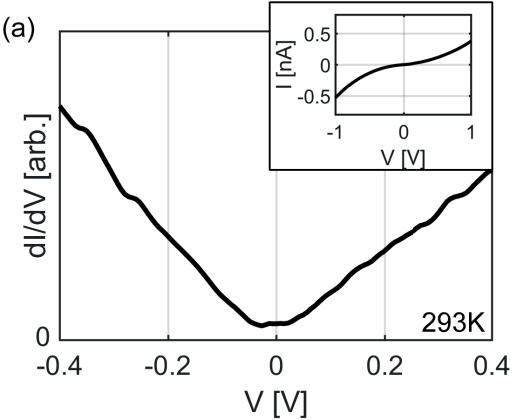

(a)

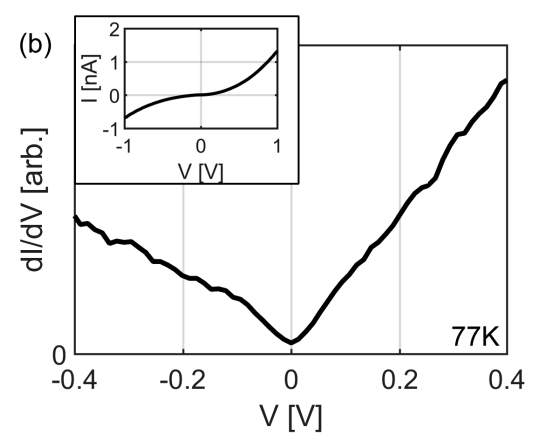

(b)

Figure 4.8 - Differential conductivity atop a typical cluster recorded at $293 \mathrm{~K}(\mathrm{a})$ and $77 \mathrm{~K}(\mathrm{~b})$. Insets show the raw $I(V)$ data. The typical V-shape, implicative of a 2D Dirac system, is clearly present for both temperatures. Measurements were performed on separate systems with separate samples which account for the difference in asymmetry and offset from the origin between the two graphs. 
V-shaped differential conductivity at $T=0 \mathrm{~K}$ can be described by

$$
\frac{d I}{d V}(V)=|a V|
$$

where $a$ is an arbitrary constant in $\mathrm{A} \mathrm{V}^{-2}$. For a finite temperature $T$ the differential conductivity has to be convoluted with the derivative of the Fermi-Dirac distribution (see appendix), resulting in

$$
\frac{d I}{d V}(V, T)=\frac{2 a k T}{q} \ln \left(2 \cosh \left(\frac{q V}{2 k T}\right)\right) .
$$

This relation is linear in both limits $V \rightarrow \pm \infty$ and in the limit $T \rightarrow 0$ the original Eq. (4.1) is recovered. In order to account for the asymmetry of the $d I / d V$ spectra as well as the non-zero density of states at the Dirac point we need to compare our results with the following expression (see appendix),

$$
\frac{d I}{d V}(V, T)=\frac{k T}{q}\left(a_{1} \ln \left(1+e^{\frac{-q\left(V-V_{0}\right)}{k T}}\right)+a_{2} \ln \left(1+e^{\frac{q\left(V-V_{0}\right)}{k T}}\right)\right)+\left(\frac{d I}{d V}\right)_{0}^{\prime}
$$
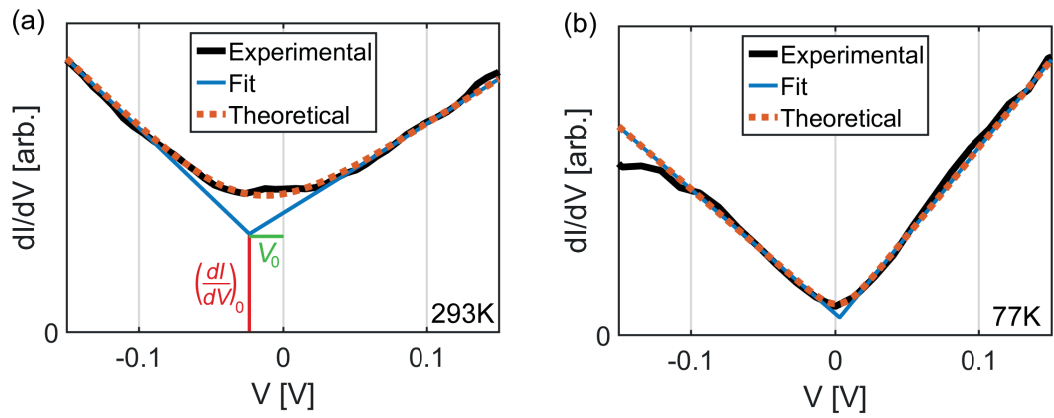

Figure 4.9 - Comparison of the experimental data (solid black), the linearly extrapolated fits (solid blue) and the theoretical thermal broadening (dotted red) at $293 \mathrm{~K}$ (a) and $77 \mathrm{~K}(\mathrm{~b})$. Horizontal and vertical offset of the projected Dirac point are illustrated in (a) through $V_{0}$ and $\left(\frac{d I}{d V}\right)_{0}$ respectively. For both temperatures a clear match between the experimental and theoretical data can be noted, suggesting only thermal effects are responsible for the broadening around the Fermi level. 
where $a_{1}$ and $a_{2}$ describe the left and right slope respectively, and $V_{0}$ and $\left(\frac{d I}{d V}\right)_{0}$ describe the horizontal and vertical Dirac point offset from the origin. Other than the asymmetry and offsets, this equation behaves similar to (4.2) in the limits $V \rightarrow \pm \infty$ and $T \rightarrow 0$. Moreover, when $a_{1}=a_{2}=a, V_{0}=0$ and $\left(\frac{d I}{d V}\right)_{0}=0$, this equation simplifies to (4.2).

The procedure to extract the parameters $a_{1}, a_{2}, V_{0}$ and $\left(\frac{d I}{d V}\right)_{0}$ from the experimental $d I / d V$ spectra is rather straightforward. Firstly, the slopes at the left and right side of the minimum of the differential conductivity are extracted from the $d I / d V$ data at voltages $\left|V-V_{0}\right|>4 k T$, where $V_{0}$ refers to the Dirac point. Secondly, the fits are extrapolated linearly in order to determine the offsets $V_{0}$ and $\left(\frac{d I}{d V}\right)_{0}$ from their intersection point, as illustrated in Fig. 4.9a. The intersecting lines now yield the $T=0 \mathrm{~K}$ differential conductivity, and the intersection point of both lines gives the Dirac point. Inserting the obtained parameters in Eq. (4.3), along with the given temperatures, yields the $d I / d V$-curves with the expected thermal broadening, also shown in Fig. 4.9.

As can be seen for the $d I / d V$ spectra at room temperature and $77 \mathrm{~K}$, a very good match between the experimental and theoretical broadening is obtained. This suggests that the only significant broadening in the experimental data results from thermal effects. The de-convoluted $d I / d V$ spectra at room temperature and $77 \mathrm{~K}$ exhibit a nearly perfect V-shape. Since the differential conductivity is only proportional to the density of states the actual values of the parameters $a_{1}$ and $a_{2}$ do not have any physical meaning. As pointed out before the asymmetry and the offset of the differential conductivity at the Dirac point are ascribed to the electronic structure of the STM tip and/or the underlying metallic substrate.

In addition, we have also studied the effect of the inclusion of a small band gap in Eq. (4.3). This is important since it is predicted that free-standing germanene has a spin-orbit gap of about $24 \mathrm{meV}$. We should, however, emphasize that the buckling of our germanene coating is substantially lower than that of freestanding germanene and therefore a much smaller spinorbit gap is expected. The quality of the fits for the differential conductivity data recorded at $77 \mathrm{~K}$ remains unaltered for band gaps smaller than $\sim 6 \mathrm{meV}$, however for values exceeding $6 \mathrm{meV}$ a substantially worse agreement with the experimental data is found.

The V-shaped differential conductivity of the germanene layer shows that the density of states scales linearly with energy, however the dispersion relation cannot be extracted from these experiments. Unfortunately, the germanene coated $\mathrm{Ge}_{2} \mathrm{Pt}$ clusters are too small for angle-resolved photoemission experiments. Alternatively the energy dispersion relation can be extracted via quasiparticle interference experiments. Electrons in the vicinity of the Fermi level are scattered at defects and edges resulting in interference patterns of incoming and outgoing electron waves, so-called Friedel oscillations. By varying the sample bias, and 
thus the energy of the electrons, the energy dispersion relation can be determined.

Figure 4.10 shows an overview of STM recorded data on top of a flat-topped cluster. In Fig. 4.10a the topography is shown, revealing the presence step edges and a small protrusion. The highlighted area shows the mapped area shown in Figure $4.10 \mathrm{~b}$. Considering Figs. $4.10 \mathrm{~b}$ and $4.10 \mathrm{c}$ we can clearly note a difference in the density of states between the center and the edge of a cluster, where the latter shows significantly lower values. This edge can thus be considered as a defect, which would normally give rise to Friedel oscillations. The expected wavelength for Friedel oscillations in map in Fig. 4.10b is given by

$$
E=\hbar v_{F} k \text { with } k=\frac{2 \pi}{\lambda}
$$

where $v_{F}$ is the Fermi velocity for germanene, which has been calculated to be around $3.8 \times 10^{5} \mathrm{~m} \mathrm{~s}^{-1}$ [?], $\hbar$ is the reduced Planck constant and $E$ is the energy of the electrons. At an energy of $0.15 \mathrm{eV}$, using Eq. (4.4), one find a wavelength of $\lambda \approx 10 \mathrm{~nm}$. However, oscillations with this wavelength are not present in Fig. 4.10b. Moreover, none of the maps we produced, at any voltage, show any signature of oscillations which could be related to defect scattering. Interestingly, for the closely related system silicene/Ag(111) Feng et al. [?] found well-defined Friedel oscillations. However, our findings are in perfect agreement with results reported by Mallet et al. [?] for epitaxial graphene grown on SiC(0001). They report that no Friedel oscillations are found on monolayer graphene, whereas
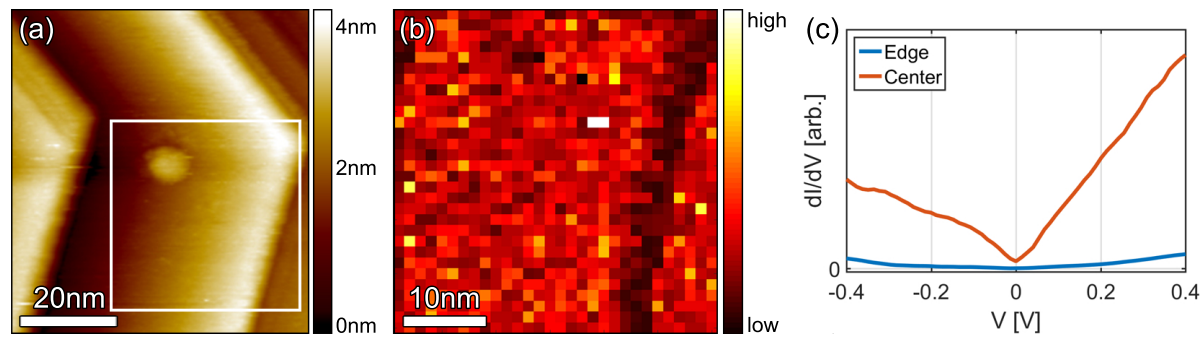

Figure 4.10 - Overview of STM recorded data at the edge atop a flat cluster at $77 \mathrm{~K}$. (a) was recorded with sample bias $-1.5 \mathrm{~V}$ and current set-point $0.4 \mathrm{nA}$. The highlighted box shows the area where the differential conductivity map in (b) was measured. This map shows the extracted $d I / d V$ at $150 \mathrm{mV}$. The edge of the cluster is clearly visible as a darker area along the right side of the image. Upon averaging the individual curves, sorted for cluster edge and center positions, the $d I / d V$ curves in (c) are obtained. There is a distinct difference in differential conductivity when comparing the cluster center to the edge, where the latter shows significantly lower values. A defect like this would normally give rise to Friedel oscillations, which are clearly absent in the measurements. The lack of these oscillations suggest the system behaves like a 2D Dirac system. 
well-resolved Friedel oscillation are observed for bilayer graphene.

As has been suggested in ref. [?] for graphene, electrons with a near-perpendicular incidence in a Dirac fermion system typically tunnel through quantum mechanical barriers in a Klein-like tunneling fashion. These electrons are also the electrons typically responsible for the largest contribution to the Friedel oscillations, since these oscillations occur in the normal direction of any 0D or 1D impurity. So the lack hereof further suggests that the presented system behaves like a helical Dirac Fermion system.

We have studied the temperature dependence of the density of states of germanene grown on $\mathrm{Ge}_{2} \mathrm{Pt}$ crystals. After the correction of thermal broadening we found a virtually perfect $\mathrm{V}$-shaped density of states, which is a hallmark of a two-dimensional Dirac system. We did not find any evidence for the presence of Friedel oscillations, which implies that the backscattering of electrons at edges and defect is suppressed. This suppression of backscattering of electrons could be explained by Klein tunneling [?]. A quantum mechanical particle with energy $\mathrm{E}$ has a non-zero probability to overcome a potential barrier $U$ that is larger than E (tunnelling). For nonrelativistic particles, the transmission probability decays exponentially with the height and the width of the barrier. In the case of two-dimensional Dirac fermion materials, however, the transmission probability for electrons that incident normally is always equal to unity independent of the actual height and width of the barrier.

\subsection{The future of germanene}

\subsubsection{Anomalous quantum Hall effect}

The electrons in the vicinity of the Dirac points in free-standing germanene are described by the relativistic variant of the Schrödinger equation, the Dirac equation [?,?,?,?,?]. A standard tight binding calculation reveals that there are two conical bands at the $\mathrm{K}$ and $\mathrm{K}^{\prime}$-points of the Brillouin zone, respectively. The dispersion relation is linear, i.e. $E=v_{F} \hbar k$, where $v_{F}$ is the Fermi velocity and $\hbar$ the reduced Planck constant. The Dirac cones lead to a zero density of states at the Fermi level and a linear in energy density of states away from the Fermi level. The peculiar electronic structure of two-dimensional Dirac materials leads to a number of intriguing physical properties. As already pointed out in the introduction the anomalous quantum Hall effect is one of these intriguing properties. For a conventional two-dimensional electron gas the Landau levels are equidistant and the separation between consecutive levels is given by $\hbar \omega_{c}$, where $\omega_{c}=e B / m$ is the cyclotron frequency with B a magnetic field normal to the two-dimensional system. For a two-dimensional Dirac system, however, the energy spectrum is 
given by $\pm v_{F} \sqrt{n \hbar e B n}(\mathrm{n}=0,1,2)$. Importantly, zero-energy Landau level $(\mathrm{n}=0)$ exists which is topologically protected with respect to possible inhomogeneity of magnetic field (or pseudomagnetic field created by deformations) [?]. Since this level is equally shared by electrons and holes the Hall conductivity per channel turns out to be half-integer (in the units of $e^{2} / h$ ) instead of normal integer quantization. This anomalous quantum Hall effect is one of the most powerful tests to check whether a two-dimensional material is indeed a Dirac fermion system.

\subsubsection{Quantum spin Hall effect}

One of the most appealing properties of germanene is its large spin-orbit gap of about $24 \mathrm{meV}$. The latter implies that this two-dimensional material is the ideal candidate for the observation of the quantum spin Hall effect (QSHE). The QSHE is of broad interest because of its scientific importance as a novel quantum state of matter and its potential for technological applications in the fields of spintronics, valleytronics and quantum computation.

In a conventional quantum Hall system the applied external magnetic field causes the electrons to move in well-defined circles. The electrons, which all orbit in the same clock (or anti-clock) wise direction, bounce back at the edges of the sample leading to a net flow of current along the edges of the sample. This current flows in one direction, and therefore, no back-scattering can occur resulting in a dissipation-less flow of charge. In contrast to the quantum Hall effect, the quantum spin Hall effect does not require an external magnetic field [?,?]. The spin-orbit coupling leads to an internal magnetic field that couples to the spin of the electrons. This asymmetry will result into two spin-polarized conduction channels at the edges of the two-dimensional material that propagate in opposite directions, the so-called gapless helical edge modes. The QSHE is therefore characterized by a vanishing charge Hall conductance and a quantized spin Hall conductance of $2 e / 4 \pi$ (an electron with charge $e$ carries a spin $\hbar / 2$ and therefore the spin Hall conductance becomes $\left.\left(2 e^{2} / h\right) *(h / e)=2 e / 4 \pi\right)$.

The QSHE in two-dimensional materials was first proposed by Kane and Mele for graphene in 2005 [?, ?], however due to the very small spin-orbit coupling in graphene extremely low temperatures are required for the realization of the QSHE state. In 2006 Bernevig, Hughes and Zhang predicted that the QSHE can also occur in $\mathrm{CdTe} / \mathrm{HgTe} / \mathrm{CdTe}$ quantum wells [?]. By varying the thickness of the quantum well, the band structure can be switched from a normal to an inverted type at a critical thickness. Shortly after this prediction the QSHE state in a $\mathrm{CdTe} / \mathrm{HgTe} / \mathrm{CdTe}$ quantum well was experimentally confirmed by König et al. [?]. These authors convincingly demonstrated at low-temperatures $(\mathrm{T}<1.4 \mathrm{~K})$ the presence of an edge conductance $2 e^{2} / h$ that only exists beyond the critical layer thickness of the quantum well. 
The spin-orbit coupling depends on the atomic number and therefore silicene and germanene, which exhibit spin-orbit gaps of $1.55 \mathrm{meV}$ and $23.9 \mathrm{meV}$, respectively, are very attractive two-dimensional materials regarding the possible observation of the QSHE. Germanene is particularly appealing because it would allow to observe the QSHE at temperatures near room temperature. The most straightforward test to check the presence of the topological protected edge modes, which is one of the hallmarks of the QSHE, is by making a spatial map of the differential conductivity at zero bias $\left((d I / d V)_{V=0}\right)$. In the interior of the two-dimensional topological insulator there will be a spin-orbit gap, whereas this gap closes at the edges of the two-dimensional material. Spatial maps of the differential conductivity can be obtained by recording IV traces using scanning tunnelling microscopy with the feedback disabled. Alternatively the differential conductivity can be obtained directly by adding a small sinusoidal voltage to the sample bias and subsequently measure the differential conductivity using a lock in amplifier.

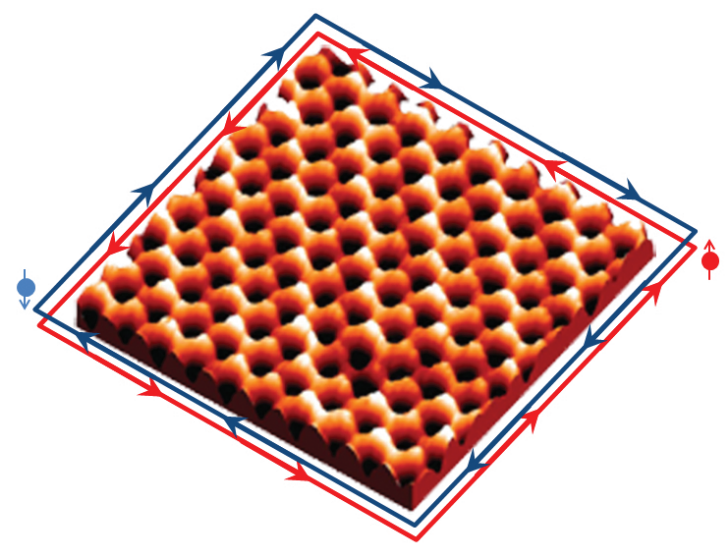

Figure 4.11 - Schematic diagram of the quantum spin Hall effect for a two-dimensional material. The quantum spin Hall effect is characterized by topological protected gapless helical edge modes that have a vanishing charge Hall conductance and a quantized spin Hall conductance.

As discussed above the QSHE is characterized by a quantized spin Hall conductance and a vanishing charge Hall conductance. In the absence of an external bias the spin-up current that flows in one direction along the edges is fully cancelled by the spin-down current that flows in the opposite direction (see Fig. 4.11). The latter does not hold for the spin current, because spin-up and spin-down currents that flow in opposite directions add up and therefore the total spin conductance is $2 e / 4 \pi$ [?]. Upon the application of an external bias the quasi Fermi levels of the left and right propagating spin-up and spin-down electrons are not equal anymore and this results into a net flow of electrons with conductance $e^{2} / h$. At the opposite edge the position of the quasi Fermi levels are reversed and therefore also here we have a net flow of electrons (now in the same direction) with 
conductance $e^{2} / h$. The total edge charge conductance in case of an applied bias is therefore $e^{2} / h+e^{2} / h=2 e^{2} / h$.

By using a multi-probe scanning tunneling microscope the edge conductance can be measured as a function of temperature as well as the number and separation of the probes. The separation between the probes should be smaller than the elastic mean free path of the charge carriers $\left(\lambda_{M F P} \approx 1000 \mathrm{~nm}\right.$ for a charge carrier mobility of $10^{5} \mathrm{~cm}^{2} / \mathrm{Vs}$ ). It should be emphasized here that the number of probes could affect the transport measurement. A straightforward LandauerBüttiker analysis reveals that the four-terminal conductance is given by $G_{14,23}=$ $I_{14} / V_{23}=2 e^{2} / h$, whereas the two-terminal conductance is $G_{14,14}=I_{14} / V_{14}=$ $2 e^{2} / 3 h$ [?]. The separate positioning of four scanning tunnelling microscope tips is far from trivial, however Baringhaus et al. [?] recently demonstrated that the edge conductance of graphene nanoribbons can be measured using this method.

\subsubsection{Opening of a band gap in germanene}

In order to open a band gap in germanene or silicene charge should be transferred from one sub-lattice to the other sub-lattice. Due to the buckling it is easier to open a band gap in germanene and silicene than in their planar counterpart graphene [?,?]. The band gap at $\mathrm{K}$ and $\mathrm{K}^{\prime}$ points of the Brillouin zone of germanene upon the application of an electric field with the voltage $\mathrm{V}$ in a direction normal to the germanene sheet is given by,

$$
E_{\sigma}(k)= \pm \sqrt{\left(v_{F} \hbar k\right)^{2}+\left(\Delta_{S O}+\frac{1}{2} \zeta \sigma e V\right)^{2}}
$$

where $\sigma= \pm 1$ refers to the spin and $\zeta= \pm 1$ to the $\mathrm{K}$ and $\mathrm{K}$ points, respectively. $\Delta_{S O}$ denotes the spin-orbit coupling. From this equation it immediately follows that there are two branches, one with a band gap $\left|\Delta_{S O}+\frac{1}{2} \zeta \sigma e V\right|$ and another with a band gap $\left|\Delta_{S O}-\frac{1}{2} \zeta \sigma e V\right|$. At the critical field, i.e. $\left|\Delta_{S O}=\frac{1}{2} \sigma e V\right|$, the smaller gap closes resulting in a transition from a topological insulator to a semi metal. For electric fields larger than the critical field germanene becomes a normal band insulator. Thus, upon increasing the electric field the gap of germanene first closes and then opens again (see Fig. 4.12 for a simple schematic diagram).

Besides the interesting topological aspects of the band gap opening in germanene it also opens the door to the realization of germanene based field-effect devices, such as for instance a transistor [?,?]. One should realize that for a proper operation of such a germanene based field-effect transistor a band gap opening of at least $300-400 \mathrm{meV}$ is required.

There are several ways to open a band gap in germanene via the transfer charge from one sub-lattice to the other. As has been shown in the preceding paragraph 


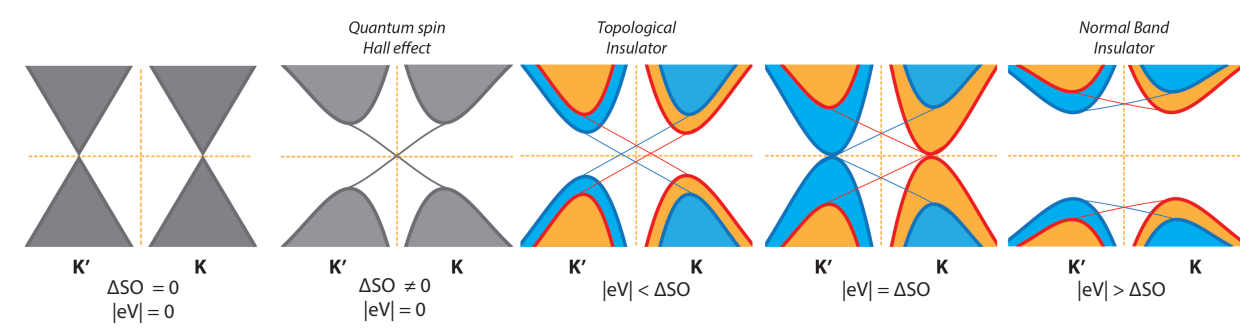

Figure 4.12 - Schematic diagram of the effect of an external electric field on the electronic structure near the Dirac point. Dirac cones at the $\mathrm{K}$ and $\mathrm{K}^{\prime}$ points of the Brillouin zone.

one way to realize this charge transfer is by applying an external electric field in a direction perpendicular to the germanene. For graphene the application of an electric field does not result into the opening of gap because graphene is completely flat and so the electric field only leads to a shift of the potential. For silicene Ezawa [?,?,?,?] and Drummond et al. [?] have predicted that silicene undergoes an interesting topological phase transition as a function of the applied electrical field. In the absence of an electric field silicene is a $Z_{2}$ topological insulator. With increasing applied electric field the band gap first closes (at a critical electric field, $E_{c}$, of $20 \mathrm{meV} / \AA$ ), and subsequently the gap opens again (the gap opening increases linearly with applied electric field). At the critical electric field there is a gapless spin-up band at the K point of the Brillouin zone, whilst the spin-down band is gapless at the $K^{\prime}$ point of the Brillouin zone. For electric fields larger than $E_{c}$ there are no gapless edge modes and thus we are dealing with a normal band insulator. For electric fields exceeding $\approx 0.5 \mathrm{~V} / \AA$ the calculations of Drummond et al. [?] revealed that the conduction band at the $\Gamma$-point and the valence band at the $\mathrm{K}$ point start to overlap leading to semi-metallic behavior of silicene. Given the similarity between silicene and germanene one expects a similar behavior for germanene, albeit the external applied electric field that is required to close the spin-orbit gap is larger for germanene [?].

One could also try to manipulate the charger transfer between the two sublattices via chemical ways, for instance via the adsorption or intercalation of foreign molecules or via coupling with a substrate. The drawback of these chemical methods is, however, that the electronic band structure of germanene might be severely affected. In case one aims at field-effect applications of germanene it is not a problem that the Dirac nature of the material is lost, but it is much more important that the modification of the electronic structure does not lead to a severe degradation of the charge carrier mobilities. Another route to manipulate the electronic structure is to apply an external strain [?], possibly in combination with the application of an external electric field [?].

For silicene Quhe et al. [?] have predicted that band gap openings as large as $0.5 \mathrm{eV}$ can be realized via the adsorption of alkali atoms on one side of the 
silicene sheet. A band gap of $0.5 \mathrm{eV}$ would result into an on/off ratio of $10^{8}$, which meets the requirement for field-effect based applications. Using density functional theory calculations $\mathrm{Ni}$ et al. [?] showed that a band gap in silicene can also be opened by the adsorption of metal atoms, such as $\mathrm{Cu}, \mathrm{Ag}, \mathrm{Au}, \mathrm{Ir}$ and Pt. Some of these metals give rise to n-type doping $(\mathrm{Cu}, \mathrm{Ag}$ and $\mathrm{Au})$, whereas Ir results into p-type doping and Pt has hardly any effect on the doping level of the silicene. Ye et al. [?] showed that a sizeable band gap in germanene can be realized by the adsorption of alkali metal atoms [?]. They claim that the band gap can be tuned from $0.02 \mathrm{eV}$ to $0.31 \mathrm{eV}$ by varying the coverage of adsorbed alkali atoms. The effective masses of the electrons and holes near the $\mathrm{K}$ and $\mathrm{K}^{\prime}$ points of the Brillouin zone after the adsorption of alkali atoms are relatively small and therefore the carrier mobilities are expected to be affected only marginally. Since germanene has only just recently been synthesized experimental studies of the tuning of germanenes band gap that could test the abovementioned theoretical predictions are not yet available.

As a final remark we would like to mention that germanene nanoribbons also have a band gap and therefore these nanoribbons can serve as the basis for a field-effect transistor [?]. However, in order to realize band gaps in germanene in the range of $0.3-0.5 \mathrm{eV}$ the nanoribbons should have a width of only a few atoms, which renders this scenario very challenging from an experimental point of view. For germanenes counterpart, i.e. silicene, a number of possible scenarios to realize a silicene-based field-effect based transistor have already been put forward [?,?,?,?]. Early 2015 the realization of the first silicene transistor was reported by Tao et al. [?]. Despite the fact that the lifetime of this silicene transistor was only a few minutes the achievements of Tao et al. are impressive given the challenging experimental hurdles that they had to overcome.

\subsection{Outlook}

In summary, germanenes debut has been impressive, but there are still many aspects that require further study. The first point of concern is to validate that the reported honeycomb lattices are indeed composed of germanium atoms. The second, and equally important, point of concern deals with the electronic structure of the germanene layers. To date there is no experimental evidence whatsoever that the germanene layers that have been synthesized so far are indeed two-dimensional Dirac systems. Since it is of utmost importance to decouple the relevant electronic states of the germanene layer from the underlying substrate the most straightforward approach is to synthesize germanene on wide band gap materials. Due to the nearly perfect lattice match with germanene, $\mathrm{h}-\mathrm{BN}$ is a possible substrate. Once it has experimentally been settled that the synthesized 
germanene is indeed a two-dimensional Dirac material several very intriguing and interesting experiments, such as the quantum spin Hall effect test and the opening of the band gap of germanene come within reach. 



\section{Appendix B}

\section{B.1 Derivation of temperature dependent dI/dV equa- tion}

The finite temperature asymmetrical linear differential conductivity can be determined through a convolution of the zero temperature asymmetrical $d I / d V$ and the derivative of the Fermi-Dirac function. The former is given by

$$
\frac{d I}{d V}(V)= \begin{cases}\left|a_{1} V\right| & \text { for } V \leq 0 \\ \left|a_{2} V\right| & \text { for } V>0\end{cases}
$$

where, similar to (4.1), $a_{1}$ and $a_{2}$ are arbitrary constants in $\mathrm{A} \mathrm{V}^{-2}$. The piecewise convolution can be described through

$$
\frac{d I}{d V}(V, T)=\int_{-\infty}^{-V}\left|a_{1}\left(V+V_{x}\right)\right| \frac{\partial f}{\partial V}\left(V_{x}, T\right) d V_{x}+\int_{-V}^{\infty}\left|a_{2}\left(V+V_{x}\right)\right| \frac{\partial f}{\partial V}\left(V_{x}, T\right) d V_{x}
$$

The derivative of the Fermi-Dirac function is given by

$$
\frac{\partial f}{\partial V}(V, T)=\frac{d}{d V} \frac{1}{e^{\frac{-q V}{k T}}+1}=\frac{q}{4 k T} \operatorname{sech}^{2}\left(\frac{q V}{2 k T}\right),
$$

where $q$ is the elementary charge and $k$ is the Boltzmann constant. Now we solve each domain of (A.2) separately, then combine the results. The first domain 
$\langle-\infty,-V\rangle$ is given by

$$
\begin{aligned}
& \int_{-\infty}^{-V}\left|a_{1}\left(V+V_{x}\right)\right| \frac{q}{4 k T} \operatorname{sech}^{2}\left(\frac{q V_{x}}{2 k T}\right) d V_{x} \\
& =-\int_{-\infty}^{-V} a_{1}\left(V+V_{x}\right) \frac{q}{4 k T} \operatorname{sech}^{2}\left(\frac{q V_{x}}{2 k T}\right) d V_{x} \\
& =-\frac{a_{1}}{4}\left[2\left(V+V_{x}\right) \tanh \left(\frac{q V_{x}}{2 k T}\right)-\frac{4 k T}{q} \ln \left(\cosh \left(\frac{q V_{x}}{2 k T}\right)\right)\right]_{-\infty}^{-V} \\
& =\frac{a_{1} k T}{q} \ln \left(2 \cosh \left(\frac{q V}{2 k T}\right)\right)-\frac{a_{1} V}{2} .
\end{aligned}
$$

Similarly for the second domain $\langle-V, \infty\rangle$ we see

$$
\begin{aligned}
& \int_{-V}^{\infty}\left|a_{2}\left(V+V_{x}\right)\right| \frac{q}{4 k T} \operatorname{sech}^{2}\left(\frac{q V_{x}}{2 k T}\right) d V_{x} \\
& =\int_{-V}^{\infty} a_{2}\left(V+V_{x}\right) \frac{q}{4 k T} \operatorname{sech}^{2}\left(\frac{q V_{x}}{2 k T}\right) d V_{x} \\
& =\frac{a_{2} k T}{q} \ln \left(2 \cosh \left(\frac{q V}{2 k T}\right)\right)+\frac{a_{2} V}{2} .
\end{aligned}
$$

Combining (A.4) and (A.5) yields

$$
\frac{d I}{d V}(V, T)=\frac{a_{1} k T}{q} \ln \left(2 \cosh \left(\frac{q V}{2 k T}\right)\right)-\frac{a_{1} V}{2}+\frac{a_{2} k T}{q} \ln \left(2 \cosh \left(\frac{q V}{2 k T}\right)\right)+\frac{a_{2} V}{2} .
$$

Upon setting $a_{1}=a_{2}=a$, Eq. (4.2), for the fully symmetrical case, can be easily derived. For the non-symmetrical case we get

$$
\begin{aligned}
\frac{d I}{d V}(V, T) & =\frac{k T}{q}\left(a_{1} \ln \left(e^{\frac{q V}{2 k T}}+e^{\frac{-q V}{2 k T}}\right)+a_{1} \ln \left(e^{\frac{-q V}{2 k T}}\right)+a_{2} \ln \left(e^{\frac{q V}{2 k T}}+e^{\frac{-q V}{2 k T}}\right)+a_{2} \ln \left(e^{\frac{q V}{2 k T}}\right)\right) \\
& =\frac{k T}{q}\left(a_{1} \ln \left(1+e^{\frac{-q V}{k T}}\right)+a_{2} \ln \left(1+e^{\frac{q V}{k T}}\right)\right) .
\end{aligned}
$$

Accounting for possible offsets this results in the relation in (4.3). 


\section{Bibliography}

[1] K.S. Novoselov, A.K. Geim, S.V. Morozov, D. Jiang, Y. Zhang, S.V. Dubonos, I.V. Grigorieva, and R.E. Smalley. Science, 306:666-669, 2004.

[2] N.D. Mermin. Phys. Rev., 176(250), 1968.

[3] D.R. Nelson and L. Peliti. J. Physique, 48(1085), 1987.

[4] A. Fasolino, J.H. Los, and M.I. Katsnelson. Nature Mat., 6(858), 2007.

[5] M.I. Katsnelson and A. Fasolino. Acc. of Chem. Res., 46(97), 2013.

[6] M.I. Katsnelson. Phys. Rev., 71(622), 1947.

[7] S.V. Morozov, K.S. Novoselov, M.I. Katsnelson, F. Schedin, L.A. Ponomarenko, D. Jiang, and A.K. Geim. Phys. Rev. Lett., 97(016801), 2006.

[8] F.P. Bundy. The Journal of Chem. Phys., 41:3809, 1964.

[9] F.P. Bundy. Physica A, 156(169), 1989.

[10] K. Takeda and K. Shiraishi. Phys. Rev. B., 50(14916-14922), 1994.

[11] G.G. Guzmán-Verri and L.C. Lew Yan Voon. Phys. Rev. B., 76(075131), 2007.

[12] C.L. Kane and E.J. Mele. Phys. Rev. Lett., 95(146802), 2005.

[13] C.L. Kane and E.J. Mele. Phys. Rev. Lett., 95(226801), 2005.

[14] J.C. Boettger and S.B. Trickey. Phys. Rev. B., 75:121402, 2007.

[15] M. Gmitra, S. Konschur, C. Ertler, C. Ambrosch-Draxl, and J. Fabian. Phys. Rev. B., 80:235431, 2009.

[16] S. Abdelouahed, A. Ernst, J. Henk, I.V. Maznichenko, and I. Mertig. Phys. Rev. B., 82:125424, 2010.

[17] C-C Liu, W. Feng, and Y. Yao. Phys. Rev. Lett., 107(076802), 2011.

[18] X.-S. Ye abd Z.-G Shao, H. Zhao, L. Yang, and C.-L. Wang. RSC Advances, 4:21216, 2014. 
[19] W. Han, R.K. Kawakami, M. Gmitra, and J. Fabian. Nature Nanotechnology, 9:794-807, 2014.

[20] S. Konschuh, M. Gmitra, and J. Fabian. Phys. Rev. B., 82:245412, 2010.

[21] B. Aufray, A. Kara, S. Vizzini, H. Oughaddou, S. Léandri, B. Ealet, and G. Le Lay. Appl. Phys. Lett., 96(183102), 2010.

[22] P. de Padova, C. Quaresima, C. Ottaviani, P.M. Sheverdyaeva, P. Moras, C. Carbonne, D. Topwal, B. Olivieri, H. Oughaddou, B. Aufray, and G. Le Lay. Appl. Phys. Lett., 96(261905), 2010.

[23] B. Lalmi, H. Oughaddou, H. Enriquez, A. Kara, S. Vizzini, B. Ealet, and B. Aufray. Appl. Phys. Lett., 97(223109), 2010.

[24] P. de Padova, C. Quaresima, B. Olivieri, P. Perfetti, and G. Le Lay. Appl. Phys. Lett., 98(081909), 2011.

[25] A. Fleurence, R. Friedlein, T. Ozaki, H. Kawai, Y. Wang, and Y. YamadaTakamura. Phys. Rev. Lett., 108(245501), 2012.

[26] P. Vogt, P. De Padova, C. Quaresima, J. Avila, E. Frantzeskakis, M.C. Asensio, A. Resta, B. Ealet, and G. Le Lay. Phys. Rev. Lett., 108(155501), 2012.

[27] L. Li, S.-Z. Lu, J. Pan, Z. Qin, Y.-Q. Wang, Y. Wang, G. Cao, S. Du, and H.-J. Gao. Adv. Mat., 26(4820), 2014.

[28] M.E. Dávila, L. Xian, S. Cahangirov, and G. Le Lay. New J. of Phys., 16(095002), 2014.

[29] B. Bampoulis, L. Zhang, A. Safaei, R. van Gastel, B. Poelsema, and H.J.W. Zandvliet. J. of Phys. Cond. Mat., 26(442001), 2014.

[30] M. Derivaz, D. Dentel, R. Stephan, M.-C. Hanf, and A. Mehdaoui. Nano Lett., 15(2510), 2015.

[31] A. Acun, B. Poelsema, H.J.W. Zandvliet, and R. van Gastel. Appl. Phys. Lett., 103(263119), 2013.

[32] J. Wang, S. Deng, Z. Liu, , and Z. Liu. Natl. Sci. Rev., 2 (1)(22-39), 2015.

[33] S. Cahangirov, M. Topsakal, E. Akturk, H. Sahin, and S. Ciraci. Phys. Rev. Lett., 102(236804), 2009.

[34] M. Houssa, G. Pourtois, V.V. Afanas'ev, and A. Stesmans. Appl. Phys. Lett, 96(082111), 2010.

[35] N. J. Roome and J. D. Carey. ACS Appl. Mater. Inter., 6(7743), 2014.

[36] A. Nijamudheen, R. Bhattacharjee, S. Choudhury, and A. Datta. J. Phys. Chem. C., 119(3802), 2015. 
[37] S. Trivedi, A. Srivastava, and R. Kurchania. J. Comput. Theor. Nanosci., 11(781), 2014.

[38] Y. Cai, C.-P. Chuu, C.M. Wei, and M.Y. Chou. Phys. Rev. B., 88(245408), 2013.

[39] M. Ye, R. Quhe, J. Zheng, Z. Ni, Y. Wang, Y. Yuan, G. Tse, J. Shi, Z. Gao, and J. Lu. Physica E, 59(60), 2014.

[40] X. Li, S. Wu, S. Zhou, and Z. Zhu. Nanoscale Res. Lett., 9(110), 2014.

[41] P. Blöchl. Phys. Rev. B., 50(17953), 1994.

[42] J.P. Perdew, K. Burke, and M. Ernzerhof. Phys. Rev. Lett., 77(3865), 1996.

[43] G. Kresse and J. Furthmüller. Phys. Rev. B., 54(11169), 1996.

[44] G. Kresse and J. Joubert. Phys. Rev. B., 59(1758), 1999.

[45] C. Kamal, A. Chakrabarti, A. Banerjee, and S. K. Deb. J. Phys.: Condens. Matter, 25(085508), 2013.

[46] H. Liu, N. Han, and J. Zhao. J. Phys.: Condens. Matter, 26(475303), 2014.

[47] B. Huang, H.-X. Deng, H. Lee, M. Yoon, B. G. Sumpter, F. Liu, S. C. Smith, and S.-H. Wei. Phys. Rev. X., 4(021029), 2014.

[48] J.P. Perdew, K. Burke, and M. Ernzerhof. Phys. Rev. Lett., 77(3865), 1996.

[49] L. Hedin. Phys. Rev., 139(A796), 1965.

[50] M. Shishkin and G. Kresse. Phys. Rev. B., 74(035101), 2006.

[51] E. Hazrati, G. A. de Wijs, and G. Brocks. Phys. Rev. B., 90(155448), 2014.

[52] G. Giovannetti, P. A. Khomyakov, G. Brocks, V. M. Karpan, J. van den Brink, and P. J. Kelly. Phys. Rev. Lett., 101(026803), 2008.

[53] P. A. Khomyakov, G. Giovannetti, P. C. Rusu, G. Brocks, J. van den Brink, and P. J. Kelly. Phys. Rev. B., 79(195425), 2009.

[54] M. Bokdam, G. Brocks, M. I. Katsnelson, and Paul J. Kelly. Phys. Rev. B., 90(085415), 2014.

[55] F. Mittendorfer, A. Garhofer, J. Redinger, J. Klimeŝ, J. Harl, and G. Kresse. Phys. Rev. B., 84(201401(R)), 2011.

[56] A.N. Rudenko, F.J. Keil, M.I. Katsnelson, and A.I. Lichtenstein. Phys. Rev. B., 86(075422), 2012.

[57] T. Olsen and K. Thygesen. Phys. Rev. B., 87(075111), 2013.

[58] P. Pflugradt, L. Matthes, and F. Bechstedt. Phys. Rev. B., 89(205429), 2014. 
[59] E. Bianco, S. Butler, S. Jiang, O.D. Restrepo, W. Windl, and J.E. Golberger. ACS Nano, 7(4414), 2013.

[60] L.C. Lew Yan Voon, E. Sandberg, R.S. Aga, and A.A. Farajin. Appl. Phys. Lett., 97(163114), 2010.

[61] M. Houssa, E. Scalise, K. Sankaran, G. Pourtois, V. V. Afanasev, and A. Stesmans. Appl. Phys. Lett., 98(223107), 2011.

[62] M.Ŝvec, P. Hapala, M. Ondráĉek, P. Merino, M. Blanco-Rey, P. Mutombo, M. Vondráĉek, Y. Polyak, V. Cháb, J. A. Martín Gago, and P. Jelínek. Phys. Rev. B., 89(201412(R)), 2014.

[63] O. Gurlu, O.A.O. Adam, H.J.W. Zandvliet, and B. Poelsema. Appl. Phys. Lett., 83(4610), 2003.

[64] N. Oncel, A. van Houselt, J. Huijben, A.-S. Hallbäck, O. Gurlu, H.J.W. Zandvliet, and B. Poelsema. Phys. Rev. Lett., 95(116801), 2005.

[65] A. Safaei, B. Poelsema, H.J.W. Zandvliet, and R. van Gastel. New J. of Phys., 16(113052), 2014.

[66] A. Saedi, B. Poelsema, and H. J.W. Zandvliet. Surf. Sci., 605(507), 2011.

[67] P. Bampoulis, A. Acun, L. Zhang, and H.J.W. Zandvliet. Surf. Sci., 626(1), 2014.

[68] H.J.W. Zandvliet. Phys. Rep., 388(1), 2003.

[69] C.-L. Lin, R. Arafune, K. Kawahara, M. Kanno, N. Tsukahara, E. Minamitani, Y. Kim, M. Kawai, and N. Takagi. Phys. Rev. Lett., 110(076801), 2013.

[70] L. Zhang, P. Bampoulis, A. van Houselt, and H. J. W. Zandvliet. Appl. Phys. Lett., 107(11):111605, 2015.

[71] B. Feng, H. Li, C.-C. Liu, T.-N. Shao, P. Cheng, Y. Yao, S. Meng, L. Chen, and K. Wu. ACS Nano, 7(10):9049-9054, 2013.

[72] X. Zhou, C. Fang, W.-F. Tsai, and J. Hu. Phys. Rev. B., 80(24):245317, 2009.

[73] D. Chiappe, C. Grazianetti, G. Tallarida, M. Fanciulli, and A. Molle. Adv. Mat., 24(37):5088-5093, 2012.

[74] D. Tsoutsou, E. Xenogiannopoulou, E. Golias, P. Tsipas, and A. Dimoulas. Appl. Phys. Lett., 103(23):231604, 2013.

[75] S.K. Mahatha, P. Moras, V. Bellini, P.M. Sheverdyaeva, C. Struzzi, L. Petaccia, and C. Carbone. Phys. Rev. B., 89(20):201416, 2014.

[76] E. Cinquanta, G. Fratesi, S. dal Conte, C. Grazianetti, F. Scotognella, S. Stagira, C. Vozzi, G. Onida, and A. Molle. Phys. Rev. B., 92(16):165427, 2015. 
[77] P. Mallet, I. Brihuega, V. Cherkez, J.M. Gómez-Rodríguez, and J.-Y. Veuillen. Comptes Rendus Physique, 17(3):294-301, 2016.

[78] M. I. Katsnelson, K. S. Novoselov, and A.K. Geim. Nature Phys., 2(9):620$625,2006$.

[79] P. Dirac. Proc. R. Soc. A, 118(610-617), 1928.

[80] P.R. Wallace. Phys. Rev., 71(622), 1947.

[81] A.H. Castro Neto, F. Guinea, N.M.R. Peres, K.S. Novoselov, and A.K. Geim. Rev. Mod. Phys., 81:109-154, 2009.

[82] T.O. Wehling, A.M. Black-Schaffer, and A.V. Balatsky. Adv. Phys., 76(1), 2014.

[83] B.A. Bernevig, T.L. Hughes, and S.-C. Zhang. Science, 314(1257), 2006.

[84] M. Knig, S. Wiedmann, C. Brne, A. Roth, H. Buhmann, L.W. Molenkamp, X.-L. Qi, and S.-C. Zhang. Science, 318(766), 2007.

[85] M.Z. Hasan and C.L. Kane. Rev. Mod. Phys., 82(3045), 2010.

[86] J. Baringhaus, M. Ruan, F. Edler, A. Tejeda, M. Sicot, A. Taleb-Ibrahimi, A.-P. Li, Z. Jiang, E.H. Conrad, C. Berger, C. Tegenkamp, and W.A. de Heer. Nature, 506(349), 2014.

[87] D. Jose and A. Datta. J. Phys. Chem. C., 116(24639-24648), 2012.

[88] M. Ezawa. New J. Phys., 14(033003), 2012.

[89] H.J.W. Zandvliet. Nano Today, 9(691), 2014.

[90] L. Tao, E. Cinquanta, D. Chiappe, C. Grazianetti, M. Fanciulli, M. Dubey, A. Molle, and D. Akinwande. Nat. Nanotech., 10(227), 2015.

[91] M. Ezawa. New J. Phys., 16(065015), 2012.

[92] M. Ezawa. Eur. Phys. J. B., 86(139), 2013.

[93] M. Ezawa. Phys. Rev. Lett., 109(055502), 2012.

[94] M. Ezawa. Phys. Rev. Lett., 110(026603), 2013.

[95] N. D. Drummond, V. Z'olyomi, and V. I. Fal'ko. Phys. Rev. B., 85(075423), 2012.

[96] T.P. Kaloni, M. Modarresi, M. Tahir, M. R. Roknabadi, G. Schreckenbach, and M.S. Freund. J. Chem. Phys. C., 119(11896-11902), 2015.

[97] T.P. Kaloni and U. Schwingenschlögl. Chem. Phys. Lett., 583(137), 2013.

[98] J.A Yan, S.-P. Gao, R. Stein, and G. Coard. Phys. Rev. B., 91(245403), 2015. 
[99] R. Quhe, R. Fei, Q. Liu, J. Zheng, H. Li, C. Xu, Z. Ni, Y. Wang, D. Yu, Z. Gao, and J. Lu. Sci. Rep., 2(853), 2012.

[100] Z. Ni, H. Zhong, X. Jiang, R. Quhe, G. Luo, Y. Wang, M. Ye, J. Yang, J. Shi, and J. Lu. Nanoscale, 6(7609), 2014.

[101] W. Xia, W. Hu, Z. Li, and J. Yang. Phys. Chem. Chem. Phys., 16(22495), 2014.

[102] S. Kaneko, H. Tsuchiya, Y. Kamakura, N. Mori, and M. Ogawa. Appl. Phys. Express, 7(035102), 2014.

[103] H. Li, L. Wang, Q. Liu, J. Zheng, W.-N. Mei, Z. Gao, J. Shi, and J. Lu. Eur. Phys. J. B., 85(274), 2012.

[104] M. Ezawa. Appl. Phys. Lett., 102(172103), 2013.

[105] H. Sadeghi. J. of Nanoscience and Nanotechnology, 14(4178), 2014. 


\section{Chapter 5}

\section{Real time growth of h-BN on $\operatorname{Ir}(111)$}

\subsection{Introduction}

The discovery of graphene has stimulated the study of various two-dimensional materials like, silicene, germanene and transition metal dichalcogenides. For example, the difficulty to open a band gap in graphene hinders the application of graphene in the semiconductor industry thus driving the study of other $2 \mathrm{D}$ materials. [?,?,?,?,?,?,?,?,?]. These materials possess high electron mobility and fascinating energy dependence of their density of states. Typically, the 2Dmaterials are (epitaxially) grown on (metal)-substrates which leads to undesired coupling between the 2D-material and the metal substrate. The usage of insulating materials between the substrate and the 2D-material could solve this issue. A promising candidate to do so nowadays is hexagonal boron nitride (h-BN). $\mathrm{h}-\mathrm{BN}$ is isostructural to graphene with the key difference that the two sub-lattices are not formed by carbon atoms, but rather by boron and nitrogen atoms. This $\mathrm{BN}$-configuration gives rise to a wide band gap and to negligible reactivity with its surroundings. As a result, hexagonal boron nitride is suitable for decoupling two materials, e.g. graphene and a metal substrate. Therefore, hexagonal boron nitride is a suitable material to use as a buffer layer for other 2D-materials and a crucial role for $\mathrm{h}-\mathrm{BN}$ in heterostructural engineering of $2 \mathrm{D}$-materials is foreseen $[?, ?, ?]$.

Several ways of synthesizing h-BN have been reported, namely via mechanical exfoliation or epitaxial growth. In this study we focus on epitaxially grown h-BN. Precursor gasses such as borazine or ammonium-borane are catalytically decomposed at metal surfaces and hexagonal boron nitride nanosheets or nanomeshes 
are formed. The metal substrate dictates the BN-geometry via metal-boron and metal-nitrogen interactions and the lattice mismatch [?]. Depending on the h$\mathrm{BN} /$ metal interaction and the lattice mismatch the resulting h-BN monolayer geometry varies from strongly corrugated and perfectly rotationally aligned, for example on $\mathrm{Rh}(111)$ [?] and $\mathrm{Ru}(0001)$ [?], with their relatively large h-BN/metal interaction and lattice mismatch, to almost completely planar with distinctly different rotational domains, like on $\mathrm{Cu}(111)$ [?].

The experimental investigation of the growth of h-BN on $\operatorname{Ir}(111)$ started [?] with an in situ X-ray photoelectron spectroscopy (XPS) and thermally programmed desorption (TPD) study by Orlando et al., showing upon borazine deposition at $170 \mathrm{~K}$ a continuous dehydrogenation from $\sim 250$ up to $\sim 1000 \mathrm{~K}$, eventually ending up in the formation of an h-BN layer at $\sim 1170 \mathrm{~K}$ with a $13 \times 13 / 12 \times 12 \mathrm{~h}$ $\mathrm{BN} / \mathrm{Ir}(111)$ unit cell, which was concluded from a low energy electron diffraction (LEED) pattern. From the XPS and near-edge X-ray adsorption fine structure (NEXAFS) measurements the authors concluded the presence of h-BN regions interacting differently with the Ir substrate. Later the same group reported, using XPS, LEED and X-ray photo-electron diffraction (XPD), the formation of domains with single orientation (with the central $\mathrm{N}$ in the moiré unit cell adsorbed on top and the adjacent $\mathrm{B}$ atom in a fcc position: $\mathrm{N}_{t o p} \mathrm{~B}_{f c c}$ ) upon deposition of borazine at room temperature, followed by annealing of the sample, while high temperature CVD would result in h-BN domains with opposite orientation $\left(\mathrm{N}_{t o p} \mathrm{~B}_{f c c}\right.$ and $\mathrm{N}_{t o p} \mathrm{~B}_{h c p}$ ) [?]. Using XPS and LEED data Usachov et al. found the formation of disorderd h-BN domains when the CVD process was performed at $\sim 875 \mathrm{~K}$, while at higher temperatures the crystallinity improved and an approximately $(10 \times 10)$ moiré pattern was found. A rapid decrease of the B and N 1 s photo emission spectra was reported during heating from $\sim 1275 \mathrm{~K}$ eventually resulting in a completely quenched signal at $\sim 1435 \mathrm{~K}$. From a further angle-resolved photoemission spectroscopy (ARPES) study they found a weak chemical interaction between h-BN and $\operatorname{Ir}(111)$ and they concluded that the appearance of replicated $\pi$ and $\sigma$ bands is mainly caused by the geometrical corrugation of the h-BN layer. A scanning tunneling microscopy (STM) study by Schulz et al. [?] revealed indeed a corrugation of $\sim 0.4 \AA$, besides a modultation of the work function of $\sim 0.5$ $\mathrm{eV}$ within the superstructure unit cell. Recently, a combined Density Functional Theory (DFT), X-ray Standing Waves (XSW), LEED and STM study [?] found that the moiré consists of strongly chemisorbed valleys surrounded by weakly physisorbed hills in an approximately 12-on-11 superstructure. For h-BN grown by CVD at elevated temperatures they reported an equal mixture of the two rotational domains, while for temperature programmed growth after exposure to the precursor at room temperature almost exclusively $\mathrm{N}_{t o p} \mathrm{~B}_{h c p}$ was observed. In addition they reported a preference for B-terminated zig-zag edges [?]. Very recently similar work on the growth of h-BN on $\operatorname{Ir}(111)$ was studied with LEEM, PEEM and Auger spectroscopy by Petrović et al [?]. 


\subsection{Materials and Methods}

An Elmitec LEEM III microscope [?] with a base pressure below $1 \cdot 10^{-10} \mathrm{mbar}$, was used to study the growth of h-BN on $\operatorname{Ir}(111)$. A Ir(111) single crystal (Surface Preparation Laboratory), was cleaned by subsequent alternating cycles of argon sputtering and annealing in oxygen environment at $1300 \mathrm{~K}$, with subsequent flash annealing at $1600 \mathrm{~K}$. The deposition of borazine (Chemos $\mathrm{GmbH}$ ) was controlled with a leak-valve and the pressure was measured by an ion gauge located $10 \mathrm{~cm}$ away from the sample and inlet. All LEEM images were acquired in bright-field mode with various electron energies at various fields of view (FOV) and sample temperatures. Additionally, $\mu$ LEED-patterns were recorded by inserting an aperture of $1.4 \mu \mathrm{m}$ diameter in order to characterize local structures. In cases where intensity measurements of the spots were not necessary multiple $\mu$ LEED-patterns at different energies were recorded and superposed into one single image in order to include as many spots as possible, which is also referred to as cumulative LEED-pattern. Photoemission electron microscopy (PEEM) was carried out by using a mercury discharge lamp.

\subsection{Growth of hexagonal boron nitride}

To start the growth of $\mathrm{h}-\mathrm{BN}$, the precursor gas borazine is let into the chamber until a pressure of $3.1 \cdot 10^{-9}$ mbar is reached at $1204 \mathrm{~K}$. The development of the pressure as a function of time is given in Sec. C.2. Within a few seconds after the borazine pressure showed a significant increase, h-BN islands are observed in Fig. 5.1(b).These fragments of borazine form h-BN islands on the surface as seen in Fig. 5.1. Two different h-BN islands can be distinguished with distinct rotational orientations with a relative rotation of $180^{\circ}$. One island has a triangular shape (marked by the orange circle) while the other type is trapezoidal (marked by the blue circles).

Diffraction patterns were recorded by placing a $1.4 \mu \mathrm{m}$ illumination aperture on locations targeted by the blue and red dots (actual size of the aperture) in Fig. 5.2(a) for the triangular and trapezoidal islands respectively. On both islands a cumulative LEED pattern (not shown) was recorded from 25 to $80 \mathrm{eV}$ with steps of $0.1 \mathrm{eV}$. The patterns recorded at $36.3 \mathrm{eV}$ for the trapezoidal and triangular island are shown in Fig. 5.2(b) and (c), respectively. The threefold symmetry of the first order hBN spots is clearly visible at this energy. From the threefold symmetry in Fig. 5.2(b) and (c) it is apparent that trapezoidal and triangular domains are rotated $180^{\circ}$ with respect to each other. Since DFT identified the $\mathrm{N}_{\text {top }} \mathrm{B}_{\text {hcp }}$ (see Fig. 5.2(d)) as the energetically more favourable of the two possible orientations [?], we associate the trapezoidal islands with $\mathrm{N}_{t o p} \mathrm{~B}_{h c p}$ configuration 


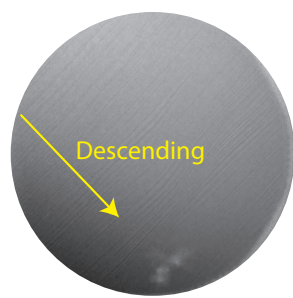

(a) $0 \mathrm{~s}-0 \mathrm{~L}$

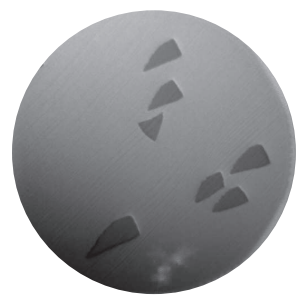

(e) $520 \mathrm{~s}$

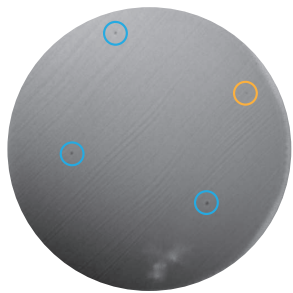

(b) $187 \mathrm{~s}$

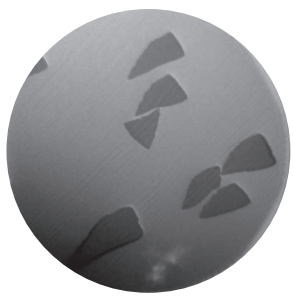

(f) $861 \mathrm{~s}$

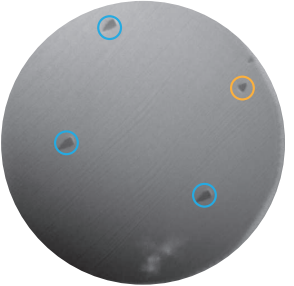

(c) $232 \mathrm{~s}$

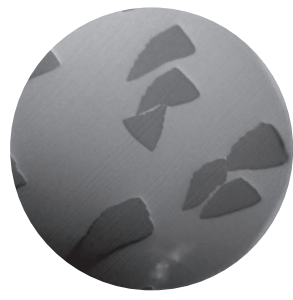

(g) $1171 \mathrm{~s}$

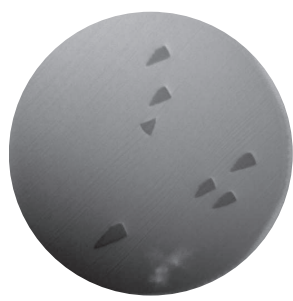

(d) $333 \mathrm{~s}$

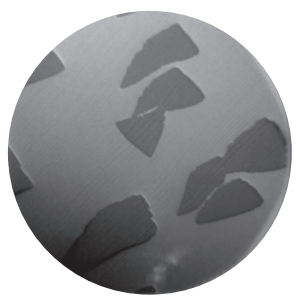

(h) $1448 \mathrm{~s}-3.3 \mathrm{~L}$

Figure 5.1 - LEEM images taken during the growth of h-BN islands with $\mathrm{FOV}=20 \mu \mathrm{m}, \mathrm{E}=16.3 \mathrm{eV}$ and at $\mathrm{T}=1204 \mathrm{~K}$. (a) The clean $\operatorname{Ir}(111)$ sample surface. The yellow arrow indicates the direction of the step edges. (b)-(h) show the growth of the h-BN islands with increasing time. The triangular island is marked by the orange circle, while trapezoidal islands are marked by the blue circles. At the end of the growth $(\mathrm{h})$, a total amount of $3.3 \mathrm{~L}$ of precursor gas borazine was let into the chamber.

and the triangular islands with the $\mathrm{N}_{t o p} \mathrm{~B}_{f c c}$ (see Fig. 5.2(e)).

From the distance between the first order h-BN and Ir spots in Fig. 5.2(b) we determine a $(11.6 \times 11.6)$ on $(10.6 \times 10.6)$ superstructure, with an associated h-BN lattice constant of $2.50 \AA$, while from Fig. $5.2(\mathrm{c})$ we arrive at a $(12.8 \times 12.8)$ on $(11.8 \times 11.8)$ superstructure, with an associated lattice constant of $2.48 \AA$. The theoretical lattice parameter of freestanding h-BN is $2.5040 \AA$ [?]. This value is close to the lattice parameter deduced from the $\mu$ LEED patterns measured on the triangular island. The exact numbers extracted from $\mu$ LEED measurements recorded at other islands and other growth temperatures differ slightly from the values discussed above.

Taking into account the $180^{\circ}$ rotational difference between the trapezoidal and triangular islands we created tentative models for these islands, see Fig. 5.3. Based on only the rotational difference between these two possible configurations one would expect that the islands are similar in shape, which is not the case. One domain is trapezoidal, the other one triangular. Moreover, the averaged size per island for the trapezoidal islands in Fig. 5.1 is larger than the size of the triangular islands. This difference can be rationalized, using the findings in Ref. [?], where with STM and DFT it was found that only zig-zag type edges occur for hBN on $\operatorname{Ir}(111)$, from which the B-terminated zig-zag edge is the energetically 


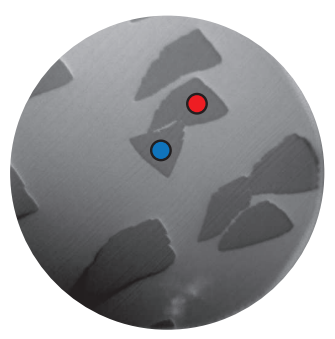

(a)

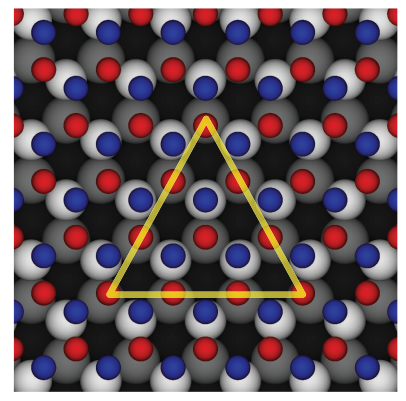

(d)

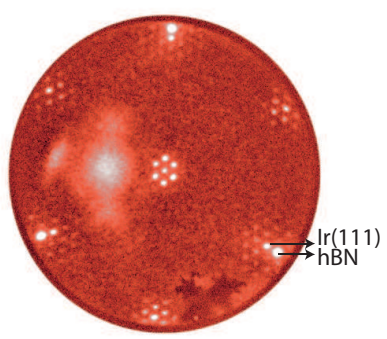

(b)

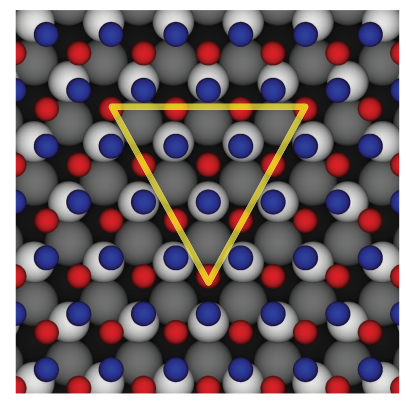

(e)

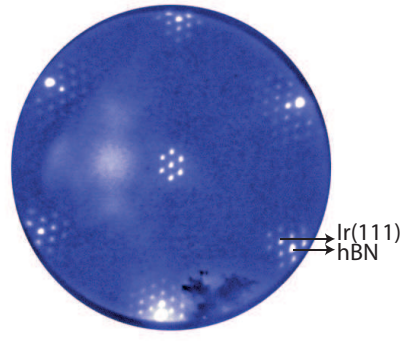

(c)

Figure 5.2 - (a) LEEM image, FOV $=20 \mu \mathrm{m}, \mathrm{E}=16.3 \mathrm{eV}$. The red and blue dots mark the position and size of the aperture used to record the $\mu$ LEED diffraction patterns for the trapezoidal / triangular island (red / blue dot) shown in panel (b) / (c). The real space models of the trapezoidal and triangular island are given in (d) and (e) respectively. The h-BN overlayer in (e) is rotated $60^{\circ}$ with respect to the overlayer depicted in (d).

most favourable one. From the images in Fig. 5.3 it is immediately clear that the triangular islands could be very well terminated by only B-terminated zigzag edges, while for the the trapezoidal islands one also needs N-termination, which is likely to be decomposed into B-terminated zigzag segments [?]. The presence of an energetically unfavorable edge termination could also explain the differences in the islands sizes. Usually this results in (kinetic) disappearance of the fast growing edges. However, if the unfavourable edge is decomposed into B-terminated zigzag segments, its growth rate is similar to the other edges and hence it would survive much longer.

Fig. 5.4 displays a PEEM image recorded after the growth of h-BN from the image series of Fig. 5.1 (FOV $150 \mu \mathrm{m}$ ). In this image 11 triangular islands (indicated by blue) and 78 trapezoidal islands (indicated by red) are observed. The triangular islands are clearly a minority, which we find consistently in all our experiments, in contrast with the observations in Ref. [?].

The intensity of the triangular islands is clearly lower than the intensity of 


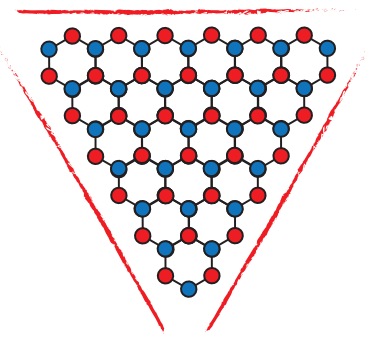

(a)

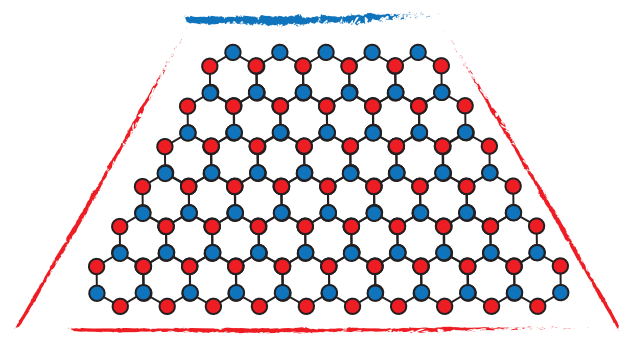

(b)

Figure 5.3 - Models for (a) triangular, (b) trapezoidal islands. Red and blue atoms denote boron and nitrogen respectively. A red stripe indicates a B-terminated zigzag edge, a blue stripe a $\mathrm{N}$-terminated zigzag edge.

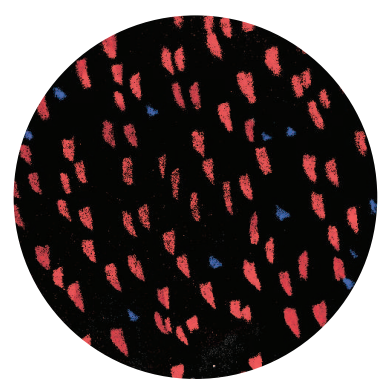

Figure 5.4 - Color coded PEEM image recorded with FOV of $150 \mu \mathrm{m}$. A total number of 89 islands are present in this image of which 78 trapezoidal islands (red) and 11 triangular islands (blue). The values used for the color coding are shown as lines in Fig. 5.5.

the trapezoidal islands, see the line profile in Fig. 5.5. The work function of clean $\operatorname{Ir}(111)$ is larger than the photon energy of the used UV-source, hence the substrate appears completely dark in all PEEM-images. The triangular islands always appear darker than the trapezoidal islands suggesting they exhibit a higher work function than the trapezoidal islands. This higher work function could be the result of a lower interaction of the triangular islands with the $\operatorname{Ir}(111)$ substrate, in accordance with the STM and DFT results in Ref. [?].

However, the ratio of triangular versus trapezoidal islands is not constant over 


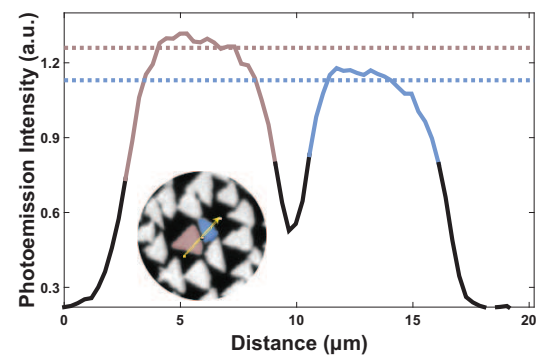

Figure 5.5 - A PEEM intensity line profile drawn through a trapezoidal (red) and triangular (blue) island. The trapezoidal island is brighter than the triangular one.

the entire sample surface, as evidenced in Fig. 5.6, where we show PEEM images on different locations of the sample. The number of triangular islands is clearly different for these different locations.

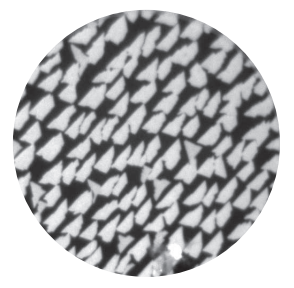

a) $[0.000 \mathrm{~mm} ; 0.000 \mathrm{~mm}]$

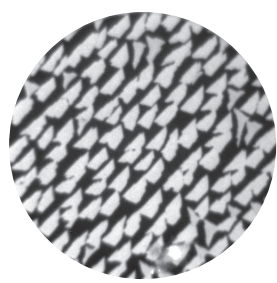

b) $[0.803 \mathrm{~mm} ; 0.000 \mathrm{~mm}]$

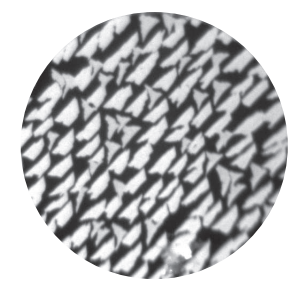

c) $[0.803 \mathrm{~mm} ;-0.789 \mathrm{~mm}]$

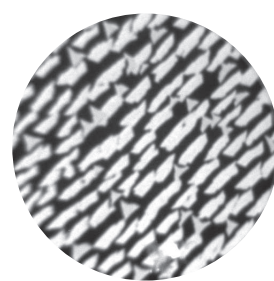

d) $[0.804 \mathrm{~mm} ; 0.808 \mathrm{~mm}]$

Figure 5.6 - PEEM images on different locations of the sample. The growth was conducted at the location of panel (a) and its coordinates have been set as a reference point for panels $(b, c, d)$. Note that the number of triangular islands is different at the different locations.

At different temperatures the growth of h-BN was monitored with PEEM and LEEM. Figure 5.7 displayes the h-BN coverage evolution in time for growth at $1248 \mathrm{~K}$ (red dots, PEEM measurement) and at $1073 \mathrm{~K}$ (blue dots, LEEM measurement). Both curves were extracted from measurements with similar borazine pressures. The thin dashed and dotted lines are fits based on the rate equation

$$
\frac{d \theta(t)}{d t}=c(1-\theta(t))
$$

assuming that borazine only sticks at the pristine Ir surface, which leads to $\theta(t)=1-e^{-c t}$. One would naively expect the growth to be faster for higher temperatures, however, the opposite is the case. This indicates that either the residence time and/or the sticking coefficient of the growth precursor, which both decrease with increasing temperature must be taken into account. For a limiting residence time at higher temperatures, only a fraction of the available free surface (directly around the nuclei) would contribute to the h-BN growth. This would considerably change the coverage versus time behaviour, especially 
for low coverages. Hence we conclude from the fact that both curves are fitted very well by Eq. 5.1, that the sticking coefficient is the growth limiting factor. A probable reason for this behaviour could be that the lifetime in the physisorption potential well is too short for the borazine molecule to rearrange itself into the geometry needed for chemisorption.

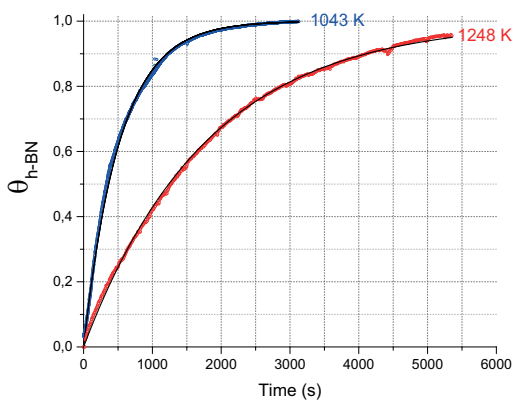

Figure $5.7-\mathrm{h}-\mathrm{BN}$ coverages during growth experiments at $1043 \mathrm{~K}$ (blue dots, $\mathrm{LEEM}, \mathrm{FOV}=20 \mu \mathrm{m}$ ) and at $1248 \mathrm{~K}$ (red dots, PEEM, FOV $=150 \mu \mathrm{m}$ ).

The solid lines are fits to Eq. 5.1.

Figure 5.8 displays the disappearance of the h-BN islands upon heating the substrate. Figure 5.8(a) displays the islands after growth at $1298 \mathrm{~K}$. Upon heating the islands disappear slowly (Fig. 5.8(b)-(d)). The triangular islands have completely vanished in Fig. 5.8(c), while the trapezoidal islands are still visible. This again indicates that the trapezoidal islands are the lowest in energy.

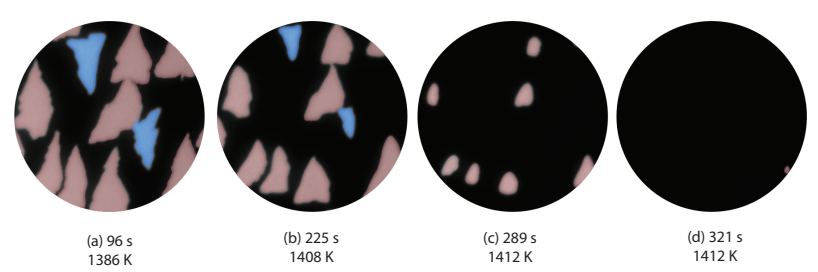

Figure 5.8 - PEEM images recorded with FOV of $100 \mu \mathrm{m}$, while the sample was slowly heated.. Different domains islands are color coded either blue (triangular) or red (trapezoidal). In panel (a) both domains are present prior to annealing. Upon raising the temperature all islands shrink (b) and the triangular (blue) islands completely decay before trapezoidal islands do (c). At last no islands remain on the surface, except for the tiny one in the lower right corner that disappeared within two seconds afterwards (d). 


\subsection{Borophene}

Figure 5.9 displays a series of LEEM images during successive h-BN growth and subsequent removal of the h-BN islands by slow heating of the crystal. In panels (b), (c) and (e) growing h-BN islands are visible, while in panels (f) and (g) they are removed. Upon a cooling the sample from panel (f) a new, elongated phase is observed in panel (h), see the red arrow, which is clearly different from the $\mathrm{h}$-BN islands. Just a second later this phase is accompanied by a brighter phase (blue arrow), which is more compact in form, while at the same time also a slightly brighter elongated phase (purple arrow) is observable in panel (i). The new phases expand during further cooling in panels (j)-(l).

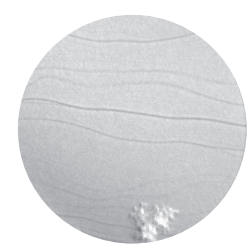

(a) $0 \mathrm{~s}-1282 \mathrm{~K}$

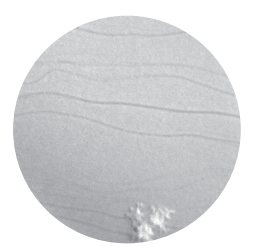

(d) $338 \mathrm{~s}-1321 \mathrm{~K}$

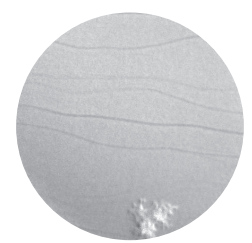

(g) $440 \mathrm{~s}-1239 \mathrm{~K}$

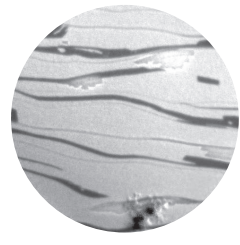

(j) $454 \mathrm{~s}-1093 \mathrm{~K}$

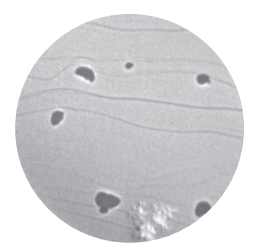

(b) $208 \mathrm{~s}-1278 \mathrm{~K}$

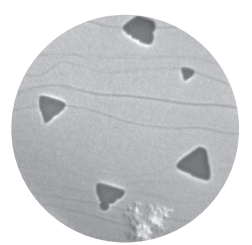

(e) $372 \mathrm{~s}-1330 \mathrm{~K}$

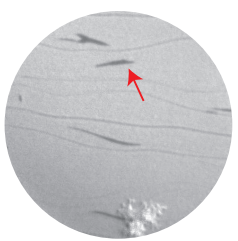

(h) $441 \mathrm{~s}-<1239 \mathrm{~K}$

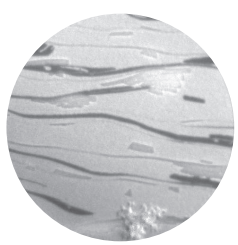

(k) $490 \mathrm{~s}-973 \mathrm{~K}$

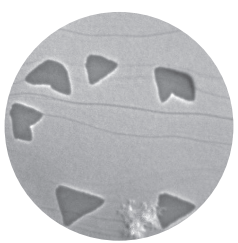

(c) $284 \mathrm{~s}-1363 \mathrm{~K}$

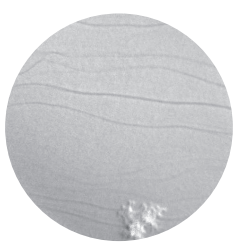

(f) $432 \mathrm{~s}-1365 \mathrm{~K}$

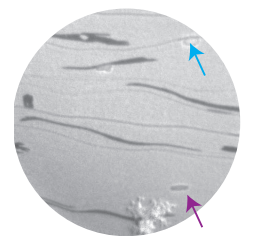

(i) $442 \mathrm{~s}-<1239 \mathrm{~K}$

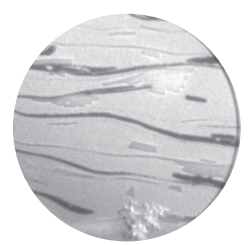

(I) $500 \mathrm{~s}-960 \mathrm{~K}$

Figure 5.9 - LEEM images of successive growth of h-BN and removal of the h-BN by increasing the temperature. FOV is $20 \mu \mathrm{m}, \mathrm{E}=16.1 \mathrm{eV}$. See text for further discussion.

This cycle can be repeated multiple times and the final coverage remains similar. Since upon heating the $\operatorname{Ir}(111)$ substrate, most likely the disintegration of the $\mathrm{h}-\mathrm{BN}$ islands results in individual boron and nitrogen atoms, we speculate that 
the boron atoms stay on the surface as adatoms or diffuse into near surface bulk at elevated temperatures, while the nitrogen atoms very likely leave the surface in the form of $\mathrm{N}_{2}$ molecules, a process which is observed well below the present temperatures [?]. Upon cooling a boron induced phase appears, visible as the elongated phases in Fig. 5.9. Providing a higher dose of borazine to the sample and repeating the cycle increases the final coverage of the new phases. The highest coverage we achieved was 64\%, see Fig. 5.10(a).

$\mu$ LEED patterns were recorded on the phases indicated with cyan and navy blue arrows as pointed out in Fig. 5.9(i,k). No additional spots were found besides the usual $\operatorname{Ir}(111)$-spots. An important note to provide here is that the phases indicated with cyan and navy blue arrows do not always occur. However, $\mu$ LEED shows a clear periodic reconstruction on the dark (elongated) stripes as indicated with the red arrow in Fig. 5.9(h). This is the phase that consistently occurs upon cooling down and which we speculate contains boron. The dark stripes always give rise to a $(6 \times 2)$ overlayer structure as shown in Fig. 5.10(b,c) and/or a superposition of the three rotational domains as shown in the appendix (Fig. C.3). It is evident that the geometry of the unit cell of this phase is not hexagonal anymore, but rather a parallelogram. A tentative real space model of this new phase is shown in Fig. 5.10(d). The yellow lines indicate the unit cell. This model is inspired by the recently described borphene structures [?,?,?,?]. Borophene is known to exhibit partly boron-atom filled hexagons and partly boron-atom empty hexagons [?, ?], which are positioned such that a $(6 \times 2)$ periodicity appears. In this configuration the majority of the boron atoms are located on either $h c p$ or $f c c$ sites with respect to the $\operatorname{Ir}(111)$ surface.

When the growth of hexagonal boron nitride is performed at temperatures in the vicinity of the segregation temperature of boron and the deposition is aborted by closing the inlet valve of borazine the new boron phase gradually evolves (Fig. 5.11(a)). Most of the time the dark stripes are attached to an h-BN island and no indication of stripes underneath or on top of h-BN islands was found. The reflected electron intensity was measured as the electron energy was varied and shown in the LEEM-IV curve in Fig. 5.11(b). The curves were measured on the (apparently) bare iridium surface (black curve), boron phase (green curve) and both the triangular (red curve) and trapezoidal (blue curve) domains of h-BN. The new "boron phase" curves clearly differ from the curves for hexagonal boron nitride or the iridium crystal. For example, the work function, i.e. the energy where the initial steep drop of electron reflectivity occurs, is much higher for the boron phase than for h-BN. Other than that, LEEM-IV spectroscopy is not yet fully understood and therefore no more details are provided in this thesis [?,?]. Note that the work function for the triangular h-BN islands is slightly lower than the one for the trapezoidal ones in Fig.5.11(b), in accordance with our PEEM observations above. 


\subsection{Borophene}

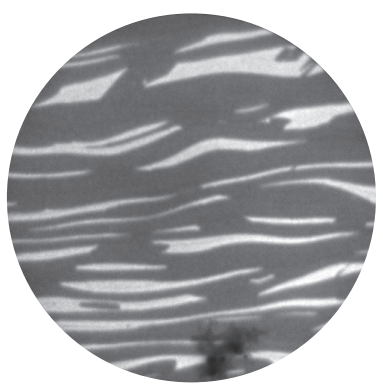

(a)

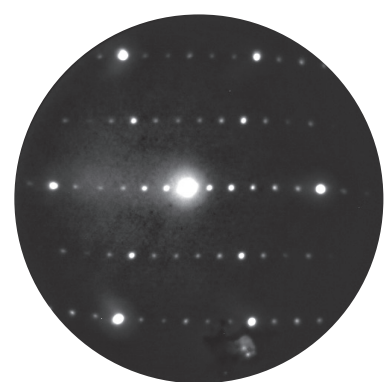

(b)

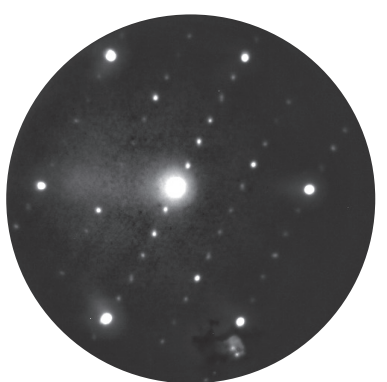

(c)

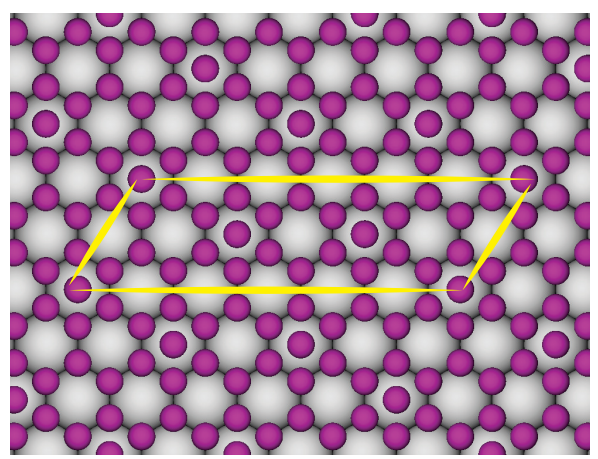

(d)

Figure 5.10 - Dark striped phases of boron on $\operatorname{Ir}(111)$ after thermal removal of h-BN and subsequently cooling down. $\mathrm{E}=16.1 \mathrm{eV}, \mathrm{FOV}=20 \mu \mathrm{m}$ (a) Cumulative LEED patterns measured from 4 to $70 \mathrm{eV}$ with steps of $0.1 \mathrm{eV}$ on two rotational domains $(b, c)$ showing a $(6 \times 2)$ phase. A tentative model of one rotational domain of borophene (d).

In literature a boron phase was recently reported on $\mathrm{Ag}(111)$-substrates [?,?,?]. In these studies a pure boron source was used to provide the substrate with boron atoms. Multiple phases were reported of which some had no clear periodicity. Those with periodicity show a rectangular phase of boron in a way similar to our own observations [?,?,?,?]. The authors of Ref. [?] have claimed their boron phases correspond to borophene, a two-dimensional Dirac material. Unlike the $s p^{2}$ hybridized materials addressed in this thesis, i.e. graphene, silicene, germanene and h-BN, borophene does not resemble these materials in the sense that the center of a hexagonal cell might contain a boron atom. The (electronic) structure of borophene is therefore much more complicated.

It is highly probable that the boron phase corresponds with borophene, but more measurements need to be performed in order to be decisive. Chemical analysis by using Auger Electron Spectroscopy, and if possible, Scanning Probe 


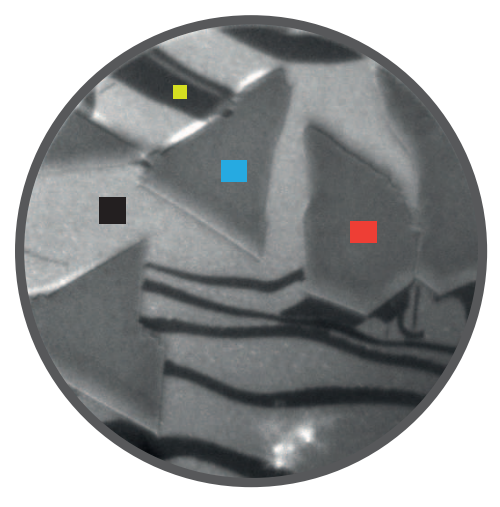

(a)

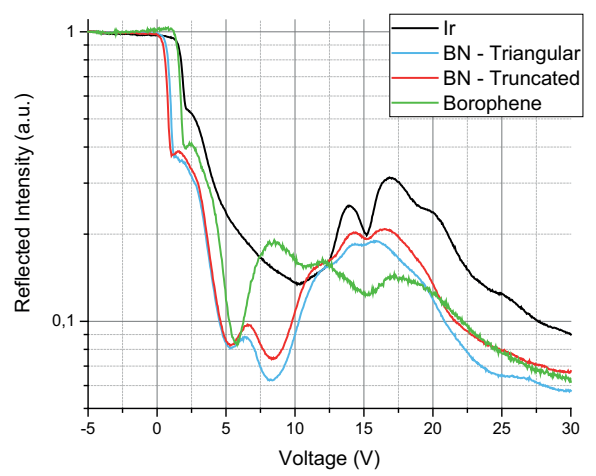

(b)

Figure 5.11 - LEEM image acquired at $668 \mathrm{~K}$ with a FOV of $25 \mu \mathrm{m}$ and electron energy of $16.4 \mathrm{eV}$. Four phases are observed, iridium (black), boron phase (yellow), triangular h-BN island (blue) and trapezoidal h-BN island (red) (a). On these phases LEEM-IV spectroscopy was recorded (b).

Microscopy might prove the growth of borophene on $\operatorname{Ir}(111)$. 


\subsection{Conclusion}

In summary, we have investigated the growth of h-BN on $\operatorname{Ir}(111)$ with LEEM, PEEM and LEED. Two distinct rotational domains of h-BN islands have been observed that have clearly different shapes, namely triangular and trapezoidal. Small variations in their respective lattice parameters are found in $\mu$ LEED patterns. Furthermore, the yield of photo-emitted electrons in PEEM is lower for the triangular island, suggesting a lower interaction between the triangular islands and the Ir substrate as compared to the trapezoidal h-BN islands. The work function depends on the "extra" dipole interaction at the interface. So, depending on the actual dipole the work function can increase or decrease. The growth of h-BN is limited by the attachment of the borazine molecules at the $\operatorname{Ir}(111)$ surface. Cooling down the sample after the thermal disintegration of $h$ $\mathrm{BN}$ by annealing the sample results in new phases that have an elongated shape. $\mu$ LEED analysis of this phase reveals a $(6 \times 2)$ superstructure. A tentative model for this phase, based on existing models for borophene is proposed. 



\section{Appendix C}

\section{C.1 Step Contrast}

The $\operatorname{Ir}(111)$-crystal possesses step edges and bunches that appear parallel to each other in our LEEM images, i.e. macroscopically (see Fig. C.1(a)). It is possible to extract which side of the step edge or step bunch is situated higher or lower. Following the recipe of Chung et al. [?] the step orientation was determined. The phase shift between two sides of a monatomic step is given by

$$
\phi=k \cdot 2 \cdot \frac{1}{3} \sqrt{3} a
$$

Here, the path length difference is incorporated in $2 \cdot \frac{1}{3} \sqrt{3} a$ (twice the monatomic step height), with a the lattice parameter of the bulk iridium crystal, e.g. $3.839 \AA$. The wave vector $k$ originates from the dispersion relation of a free electron, i.e.

$$
k=\frac{\sqrt{2 m E}}{\hbar}
$$

Substituting the latter equation in the former equation gives

$$
\phi=\sqrt{\frac{8 m}{3 \hbar^{2}}} a \cdot \sqrt{E}
$$

Finally, the reduced phase is found by

$$
\phi_{r}=\bmod \left(\frac{\phi}{2 \pi}, 1\right)
$$

The operator $\bmod (x, 1)$ is the modulo operator and finds the remainder upon division. The reduced phase is dependent on the incident electron energy. By 
recording line profiles on step edges at various incident electron energies the profiles are compared with the phase shift. From this comparison the location of the upper (or lower) terrace is determined. Figure C.1 depicts the results. Firstly, all images were recorded at under focussed conditions. The under focussing was roughly equivalent for every recorded image. Next, at several incident electron energies typically hundred images were captured and averaged afterwards. A line profile was drawn from the upper left to the lower right direction. As can be seen from Fig. C.1(a-d) at reduced phases below $\frac{1}{2}$ the blue side of the step edge appears brighter. The contrary is true for reduced phases above $\frac{1}{2}$. In under focussed conditions this means that the upper terrace is located at the blue side of the edge [?]. Figure C.1(e,f) indicate the descending step direction.

Three remarks need to be put in place here. Firstly, the energy used to calculate the phase shift does not include potentials in the thin film and is not corrected for the work functions of the sample and the $\mathrm{LaB}_{6}$-electron gun. Secondly, it is unclear whether or not the recorded steps are monatomic as imaging demands higher resolution. Thirdly, it is assumed that the reflected or diffracted waves do not have an additional phase shift.

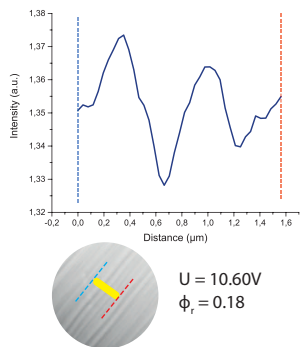

(a)

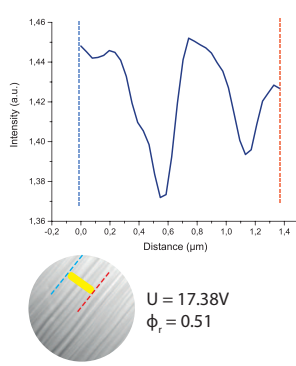

(c)

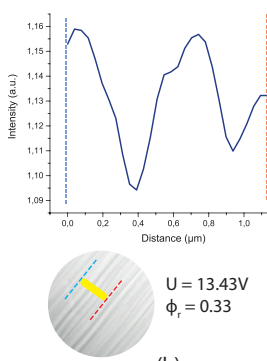

(b)

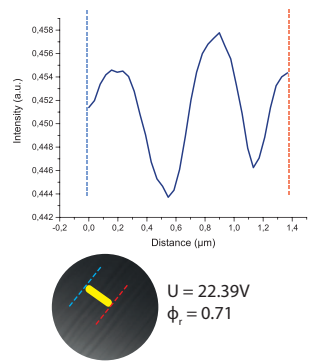

(d)

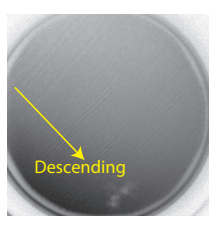

(e)

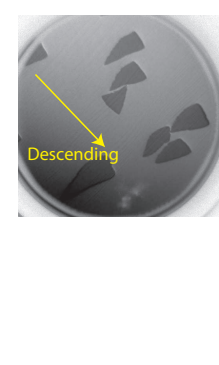

(f)

Figure C.1 - Step orientation of the $\operatorname{Ir}(111)$-crystal: panels (a-d) show line profiles measured at the same locations with different incident electron energies (and therefore reduced phase shifts). The blue side of the curve becomes dominated by the red side as the reduced phase shift is higher than 0.5. Panels (e,f) depict illustrative orientation on downward step flow. 


\section{C.2 Pressure development}

The borazine inlet pressure was monitored by an ion gauge distanced approximately $15 \mathrm{~cm}$ from the nozzle. The gas was let in by turning a valve slowly, hence it takes time to reach the desired pressure. The pressure was tracked in time and integrated in order to retrieve the dose of borazine in units of Langmuir.

\begin{tabular}{|l|l|}
\hline Time & Pressure \\
\hline$<0 \mathrm{~s}$ & $1.80 \cdot 10^{-9}$ mbar \\
\hline $0 \mathrm{~s}$ & First increase \\
\hline $30 \mathrm{~s}$ & $2.10 \cdot 10^{-9}$ mbar \\
\hline $80 \mathrm{~s}$ & $2.24 \cdot 10^{-9}$ mbar \\
\hline $112 \mathrm{~s}$ & $2.50 \cdot 10^{-9}$ mbar \\
\hline $150 \mathrm{~s}$ & $2.80 \cdot 10^{-9}$ mbar \\
\hline $170 \mathrm{~s}$ & $3.00 \cdot 10^{-9}$ mbar \\
\hline $191 \mathrm{~s}$ & $3.34 \cdot 10^{-9}$ mbar \\
\hline $220 \mathrm{~s}$ & $3.48 \cdot 10^{-9}$ mbar \\
\hline $280 \mathrm{~s}$ & $3.51 \cdot 10^{-9}$ mbar \\
\hline $350 \mathrm{~s}$ & $3.54 \cdot 10^{-9}$ mbar \\
\hline $491 \mathrm{~s}$ & $3.54 \cdot 10^{-9}$ mbar \\
\hline $1312 \mathrm{~s}$ & $3.49 \cdot 10^{-9}$ mbar \\
\hline
\end{tabular}




\section{C.3 Cold substrate deposition}

Another growth method was performed by depositing $60 \mathrm{~L}$ of borazine on the $\operatorname{Ir}(111)$-substrate at room temperature with subsequent annealing. We call this method the cold substrate deposition (CSD). At first in LEEM (and LEED) the reflected electron intensity decreases during the deposition indicating that no periodic structures of borazine molecules, e.g. molecular frameworks, are formed. Upon annealing the sample to $1040^{\circ} \mathrm{C}$ LEED patterns resembling h-BN are observed with the notion that the moire pattern is not as pronounced as before, i.e. only the first order satellite spots are visible. The LEED pattern is given in Fig. C.2(b). In this growth method the island size is expected to be small, while the island density is high. This is observed in BF-LEEM, we were not able to resolve any of these islands, since the resolution that is required to image the islands could not be achieved by our microscope. Since the island size is much smaller than the illumination aperture that is inserted in previous experiments multiple domains are recorded in $\mu$ LEED. The LEED-patterns again show threefold symmetry proving that the one of the two rotational domain is dominating.

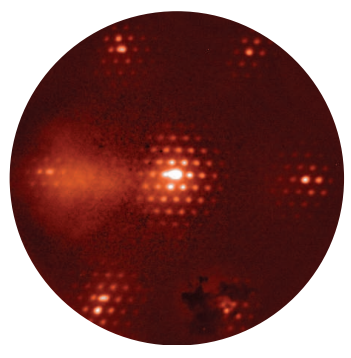

(a)

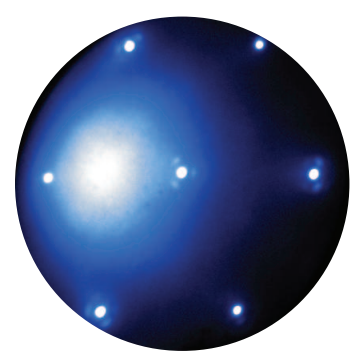

(b)

Figure C.2 - Cumulative LEED patterns from $5 \mathrm{eV}$ to $80 \mathrm{eV}$ recorded on h-BN grown by hot substrate deposition (a) and cold substrate deposition with subsequent annealing (b). 


\section{C.4 Borophene: LEED}

A superposition of three domains is given in Fig. C.3. The domains are rotated $\pm 60^{\circ}$ with respect to each other.

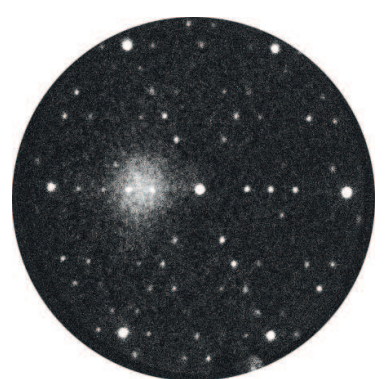

Figure C.3 - A LEED pattern recorded on dark stripes of borophene. The diffraction pattern comprises of three rotational domains of $(6 \times 2)$ each separated $\pm 60^{\circ}$ from each other. 



\section{Bibliography}

[1] K.S. Novoselov, A.K. Geim, S.V. Morozov, D. Jiang, Y. Zhang, S.V. Dubonov, I.V. Grigorieva, and R.E. Smalley. Science, 306:666-669, 2004.

[2] M.I. Katsnelson, K.S. Novoselov, and A.K. Geim. Nature Physics, 2:620-625, 2006.

[3] S. Cahangirov, M. Topsakal, E. Aktürk, H. Şahin, and S. Ciraci. Phys. Rev. Lett., 102:236804, 2009.

[4] P. Vogt, P. De Padova, C. Quaresima, E. Frantzeskakis, M.C. Asensio, A. Resta, B. Ealet, and G. Le Lay. Phys. Rev. Lett., 108:155501, 2012.

[5] A. Acun, B. Poelsema, H.J.W. Zandvliet, and R. van Gastel. Appl. Phys. Lett., 31:121312, 2013.

[6] D. Chiappe C. Grazianetti M. Fanciulli M. Dubey A. Molle L. Tao, E. Cinquanta and D. Akinwande. Nature Nanotechnology, 10:227-231, 2015.

[7] P. Bampoulis M. Farmanbar A. van Houselt A.N. Rudenko M. Lingenfelder G. Brocks B. Poelsema M.I. Katnselson A. Acun, L. Zhang and H.J.W. Zandvliet. J. Phys. Condens. Matter, 27:443002, 2015.

[8] J. Brivio V. Giacometti B. Radisavljevic, A. Radenovic and A. Kis. Nature Nanotechnology, 6:147-150, 2011.

[9] T.S. Rahman E.R. Mucciolo E. Ridolfi, D. Le and J. C.H. Lewenkopf. J. Phys. Condens. Matter, 27:365501, 2015.

[10] I. Meric C. Lee L. Wang S. Sorgenfrei K. Watanabe T. Taniguchi P. Kim K.L. Shepard C.R. Dean, A.F. Young and J. Hone. Nature Nanotechnology, 5:722, 2010.

[11] A.F. Young M. Yankowitz B.J. LeRoy K. Watanabe T. Taniguchi P. Moon M. Koshino P. Jarillo-Herrere B. Hunt, J.D. Sanchez-Yamagishi and R.C. Ashoori. Science, 340:1427, 2013. 
[12] X. Cui N. Petrone C-H. Lee M.S. Choi D-Y. Lee C. Lee W.J. Yoo K. Watanabe T. Taniguchi C. Nuckolls P. Kim G-H. Lee, Y-J. Yu and J. Hone. ACS Nano, 7(9):7931-7936, 2013.

[13] R. Koitz A. P. Seitsonen M. Iannuzzi J.G. Díaz, Y. Ding and J. Hutter. Theor. Chem. Acc., 132:1350, 2013.

[14] R. Widmer O. Groening R. Laskowski P. Blaha K. Schwarz A. Goriachko-H. Over S. Gsell M. Schreck H. Sachdev T. Greber S. Berner, M. Corso and J. Osterwalder. Angewandte Chemie, 46:5115, 2007.

[15] M. Knapp H. Over M. Corso T. Brugger S. Berner J. Osterwalder A. Goriachko, Y. He. and T. Greber. Langmuir, 23:2928-2931, 2007.

[16] R. Koitz M. Iannuzzi A.P. Seitsonen J. Hutter H. Sachdev S. VijayaraghavanF. Bischoff K. Seufert J.V. Barth S. Joshi, D. Ecija and W. Auwärter. Nano Lett., 12:5821-5828, 2012.

[17] P. Lacovig I. Boscarato A. Baraldi F. Orlando, R. Larciprete and S. Lizzit. J. Phys. Chem. C., 116:157-164, 2012.

[18] L. Omiciuolo N.G. Apostol R. Larciprete A. Baraldi F. Orlando, P. Lacovig and S. Lizzit. ACS Nano, 8(12):12063-12070, 2014.

[19] S. K. Hämäläinen T. Demonchaux A.P. Seitsonen F. Schulz, R. Drost and P. Liljeroth. Phys. Rev. B., 89:235429, 2014.

[20] F.H. Farwick Zum Hagen, D.M. Zimmermann, C.C. Silva, C. Schlüter, N. Atodiresei, W. Jolie, A.J. Martínez-Galera, D. Dombrowski, U.A. Schröder, M. Will, P. Lazic, V. Caciuc, S. Blügel, T.-L. Lee, T. Michely, and C. Busse. ACS Nano, 10(12):11012-11026, 2016.

[21] M. Petrović, U. Hagemann, M. Horn von Hoegen, and F.-J. Meyer zu Heringdorf. Applied Surface Science, 420:504-510, 2017.

[22] E. Bauer. Reg. Prog. Phys., 57:895-906, 1994.

[23] R.W. Lynch and H.G. Drickamer. The Journal of Chemical Physics, 44:181, 1966.

[24] H.H. Sawin and R.P. Merrill. The Journal of Chemical Physics, 73:996, 1980.

[25] A.J. Mannix, X.-F. Zhou, B. Kiraly, J.D. Wood, D. Alducin, B.D. Myers, X. Liu, B.L. Fisher, U. Santiago, J.R. Guest, M.J. Yacaman, A. Ponce, A.R. Oganov, M.C. Hersam, and N.P. Guisinger. Science, 350(6267):1513-1516, 2015.

[26] B. Feng, O. Sugino, R.-Y. Liu, J. Zhang, R. Yukawa, M. Kawamura, T. Iimori, H. Kim, Y. Hasegawa, H. Li, L. Chen, K. Wu, H. Kumigashira, F. Komori, T.-C. Chiang, S. Meng, and I. Matsuda. Phys. Rev. Lett., 118:096401, 2017. 
[27] B. Feng, J. Zhang, Q. Zhong, W. Li, S. Li, H. Li, P. Cheng, S. Meng, L. Chen, and K. Wu. Nature Chemistry, 8:6, 2016.

[28] Z. Zhang, A.J. Mannix, Z. Hu, B. Kiraly, N.P. Guisinger, M.C. Hersam, and B.I. Yakobson. Nanolett., 16(10):6622-6627, 2016.

[29] B. Poelsema, M.S. Altman, R. van Gastel, H.J.W. Zandvliet, and A. van Houselt. New J. of Phys., 19:013024, 2017.

[30] J. Jobst, J. Kautz, D. Geelen, R.M. Tromp, and S.J. van der Molen. Nature Communications, 6:8926, 2015.

[31] W.F. Chung and M.S. Altman. Ultramicroscopy, 74:237-246, 1998. 



\section{Summary}

In this thesis various 2D-materials have been described. The two-dimensional materials of interest possess two sub-lattices and a honeycomb structure. These ingredients together with respective $p_{z}$-orbitals overlapping in the two sublattices account for fascinating properties that are ascribed to Dirac materials. Graphene, silicene and germanene are examples of Dirac materials. Another example of a material with a comparable structure is hexagonal boron nitride. However, in this case, the two sub-lattices are comprised of two different elements, namely boron and nitrogen. Henceforth leading to different properties of which the most important ones are the wide band gap $(5.9 \mathrm{eV})$ and its chemical inertness.

In Chapter 1 the instruments, the materials and the methods were introduced. LEEM (Low Energy Electron Microscopy) was used in order to visualize the growth of silicene and hexagonal boron-nitride real-time. Furthermore, the structural properties of both materials was investigated by using low energy electron diffraction. Measurements on the electronic properties of germanene were conducted by low temperature scanning tunneling microscopy accompanied with scanning tunneling spectroscopy.

An extensive introduction to the electronic structure of 2D-materials was provided in Chapter 2. The nearest-neighbor tight-binding method was applied on pristine graphene. We showed that around the $\mathrm{K}$ and $\mathrm{K}^{\prime}$-points graphene is a semimetal of which the dispersion relation features linear properties. The band structure in the vicinity of the $\mathrm{K}$ and $\mathrm{K}^{\prime}$-points has been given the name Dirac cone. Next, we exploited the Dirac cone by using the long wavelength limit and found indeed a linear dispersion relation. By including the theory of special relativity in the Hamiltonian of the Schrödinger equation derived from the Dirac equation. Consequently, it was found that the electrons behave as massless fermions and have a Fermi velocity equal to the speed of light. The tight-binding method was applied on both zigzag and armchair graphene edges with various widths. The type of edge termination and the width significantly changed the electronic properties of the graphene edges. 
Chapter 3 described the growth of silicene on $\mathrm{Ag}(111)$. The study was performed in-situ with LEEM and $\mu$ LEED. Silicene islands nucleate and grow during the deposition of silicon. Before the silicene layer is fully closed a phase transition occurs from silicene to "sp $s p^{3}$-like" hybridized silicon upon continuing the deposition. In another experiment the phase transition was induced by increasing the substrate temperature. Both experiments clearly show that silicene is unstable. The instability of silicene on $\operatorname{Ag}(111)$ is problematic, since the $\operatorname{Ag}(111)$ was ought to be ideal due to the relatively small lattice mismatch between silicene and $\operatorname{Ag}(111)$. Furthermore, no indications for multilayer silicene formation were found in this study.

Germanene's debut is addressed in Chapter 4. A review and a STM-study are provided in this Chapter. The electronic structure of pristine germanene and bilayer germanene is discussed. An overview of experimental work considering the growth of germanene is mentioned. The fascinating properties of germanene arising from the large spin-orbit coupling is put in a perspective that could lead to phenomenal applications in future. In order to attain this possibility the pristine properties of germanene need to be achieved. H-BN is proposed as a possible substrate retrieving the properties of freestanding germanene.

The growth of h-BN on $\operatorname{Ir}(111)$ was studied with LEEM, PEEM and LEED as described in Chapter 5. Due to the two different elemental sub-lattices two distinct rotational domains of h-BN islands were observed that have clearly different shapes, e.g. trapezoidal and triangular. The two distinct types of islands have small variations in their respective lattice parameters. Additionally, our PEEM study shows that the yield of photo-emitted electrons is less for the triangular island. This indicates a lower interaction between the triangular islands and the Ir substrate, compared to the trapezoidal h-BN islands. The growth of h-BN on $\operatorname{Ir}(111)$ is limited by the sticking of the borazine molecules at the $\operatorname{Ir}(111)$ surface. H-BN was thermally disintegrated and upon cooling down new phases occurred that have a $(6 \times 2)$ superstructure. A tentative model for this elongated phase, probably borophene, was proposed.

In summary, various 2D-materials ranging from graphene, silicene, germanene, hexagonal boron-nitride and borophene have been addressed in this thesis. The large size of the h-BN islands is promising for epitaxially grown graphene, silicene or germanene on top of h-BN. This encouragement is a major deliverable of this thesis for future research and applications. 


\section{Samenvatting}

In deze thesis zijn verscheidene twee-dimensionale materialen bestudeerd. Deze twee-dimensionale materialen bezitten allen twee subroosters en een honingraatrooster. De $p_{z}$-orbitalen van de twee subroosters zijn verantwoordelijk voor de fascinerende van deze zogenaamde Dirac-materialen. Grafeen, siliceen and germaneneen zijn enkele voorbeelden van zulke Dirac-materialen. Een ander voorbeeld van een 2D-materiaal met een vergelijkbare structuur is hexagonaal boornitride. In tegenstelling tot de eerdergenoemde materialen bestaan de twee subroosters in hexagonaal boornitride uit verschillende elementen, namelijk boor en stikstof. Zodoende leidt dit tot aanzienlijk verschillende eigenschappen waarvan de belangrijkste eigenschappen de grote bandkloof $(5.9 \mathrm{eV})$ en de inertheid zijn.

In Hoofdstuk 1 worden de gebruikte meetopstellingen, de materialen en de gebruikte methodes geïntroduceerd. Lage energie elektronmicroscopie (LEEM) is gebruikt om de groei van siliceen en hexagonaal boornitride in situ af te beelden. Daarnaast zijn de structurele eigenschappen van beide materialen onderzocht door middel van lage energie elektrondiffractie (LEED). Metingen van de structurele en elektronische eigenschappen van germaneen zijn uitgevoerd met behulp van rastertunnelmicroscopie (STM) en rastertunnelspectroscopie (STS).

Een inleiding in de elektronische structuur van 2D-materialen wordt gegeven in Hoofdstuk 2. The naaste-buren tight-binding methode is toegepast op vrijstaand grafeen. We laten zien dat rondom de $\mathrm{K}$ and $\mathrm{K}^{\prime}$-punten grafeen een halfmetaal is met een linearie dispersierelatie.. De bandenstructuur in de nabijheid van de $\mathrm{K}$ en $\mathrm{K}^{\prime}$-punten, gekarakteriseerd deze halfmetallische lineaire dispersierelatie, wordt ook wel de Dirac-kegel genoemd. Vervolgens hebben we de Dirac-kegel bestudeerd door de bandenstructuur te benaderen voor grote golflengten. Hieruit volgt inderdaad een lineaire dispersierelatie. Door het gebruik van de speciale relativiteitstheorie in de gebruikelijke Hamiltoniaan van de Schrödingervergelijking is de Diracvergelijking afgeleid. Daarbij werd gevonden dat de $p_{z}$-elektronen in grafeen zich gedragen als massaloze fermionen met als de Fermisnelheid de lichtsnelheid. Tevens is de tight-binding methode 
toegepast op grafeenranden door de terminatie en de breedte te variëren waarbij noemenswaardige veranderingen van de elektronische eigenschappen zijn voorspeld.

Hoofdstuk 3 gaat over de groei van siliceen op $\mathrm{Ag}(111)$. Dit onderzoek is in-situ uitgevoerd d.m.v. LEEM en LEED. Siliceeneilanden nucleëren en groeien tijdens de depositie van silicium. Vlak voordat er een volle laag is gesloten, ontstaat er tijdens het voortzetten van de depositie echter een faseovergang van siliceen naar "sp ${ }^{3}$-achtig" gehybridiseerd silicium. In een ander experiment is een soortgelijke faseovergang geobserveerd bij het verhogen van de substraat temperatuur. Beide experimenten laten duidelijk zien dat siliceen instabiel is. Deze instabiliteit van siliceen op $\operatorname{Ag}(111)$ is problematisch, aangezien het zilversubstraat als een ideaal substraat wordt gezien door de kleine verschillen in roosterparameters van siliceen en $\operatorname{Ag}(111)$. Verder zijn er geen aanwijzingen gevonden voor de formatie van multilagen siliceen.

Germaneen is bestudeerd in Hoofdstuk 4. Eerst wordt een overzicht gegeven van de recente bechrijvingen van germaneen. De elektronische structuur van vrijstaande germaneen en van een bilaag germaneen wordt hier besproken, alsmede een overzicht van experimenten m.b.t. de groei van germaneen. De exotische eigenschappen van germaneen, en de rol van de naar verhouding grote spinbaankoppeling, worden besproken met mogelijke toepassingen in het oog. Om de toekomstige toepassingsvoorspelling waar te kunnen maken, dient men de eigenschappen van vrijstaande germaneen te verwezenlijken. Hexagonaal boornitride wordt voorgesteld als een mogelijke substraat om de eigenschappen van vrijstaande germaneen te bestuderen.

De groei van h-BN op $\operatorname{Ir}(111)$ is bestudeerd d.m.v. LEEM, LEED and fotoemissie elektronmicroscopie (PEEM) in Hoofdstuk 5. Ten gevolge van de twee verschillende elementen op de respectievelijke subroosters zijn twee verschillende rotationele domeinen van h-BN op $\operatorname{Ir}(111)$ geobserveerd. Beide soorten eilanden hebben een unieke geometrie, namelijk trapezoïdaal of driehoekig. Tevens is er een kleine variatie in de roosterparameters van beide soorten eilanden gevonden. Aansluitende PEEM-metingen laten zien dat de driehoekige eilanden een lager aantal geëmitteerde fotoelektronen heeft en een zwakkere wisselwerking met het substraat heeft. Uit de vorm van de groei-curves van h-BN op $\operatorname{Ir}(111)$ geconcludeerd dat de groei wordt beperkt door de kleefkans van borazinemoleculen op de iridiumsubstraat tijdens de depositie. H-BN valt thermisch uiteen bij verhitting en bij afkoelingen nadien ontstaan er nieuwe fasen met een $(6 \times 2)$ superstructuur. Voor deze nieuwe fase, hoogstwaarschijnlijk borofeen of een nauw verwante structuur, is een voorlopig model voorgesteld.

In dit proefschrift zijn de 2D-materialen grafeen, siliceen, germaneen en hexagonaal boornitride bestudeerd. De grote h-BN eilanden vormen een geschikte 
substraat voor epitaxiaal gegroeide grafeen, siliceen en germaneen om mogelijkerwijs eigenschappen van de Dirac-materialen te bestuderen met minder koppeling naar het substraat dan bij het gebruik van een metaalsubstraat. 



\section{Publications}

In Preparation: Hexagonal Boron Nitride and Borophene on $\operatorname{Ir}(111)$ : a real-time study by LEEM. Expected finishing draft: June 2017

Atomically Controlled Surfaces, Interfaces and Nanostructures,

A. Acun et al., Superstripes Press, Rome, Italy, ISBN: 9788866830597, 2016

Scanning Tunneling Spectroscopy study of the Dirac spectrum of germanene,

C.J. Walhout, A. Acun, L. Zhang, M. Ezawa, H.J.W. Zandvliet, Journal of Physics: Condensed Matter, 28 (28), 284006, 2016

Special issue: 2D-materials

Germanene: the germanium analogue of graphene,

A. Acun, L. Zhang, P. Bampoulis, M. Farmanbar, A. van Houselt, A.N. Rudenko, M. Lingenfelder, G. Brocks, B. Poelsema, M.I. Katsnelson, H.J.W. Zandvliet, Journal of Physics: Condensed Matter, 27 (44), 443002, 2015

Special note: in the list of most read and most cited of the mentioned journal

Wordt siliceen het nieuwe grafeen?,

A. Acun, H.J.W. Zandvliet, R. van Gastel, Nederlandse Tijdschrift voor Natuurkunde, 80 (8), 292-295, 2014Dutch Physical Society

Electronic and energetic properties of $\mathrm{Ge}(110)$ pentagons,

P. Bampoulis, A. Acun, L. Zhang, H.J.W. Zandvliet, Surface science, 626, 1-5, 2014 
Siliceen, een waardige opvolger van het wondermateriaal grafeen?,

A. Acun, B. Poelsema, H.J.W. Zandvliet, R. van Gastel, NEVAC blad, 2 (52), 6-10, 2014

Dutch Vacuum Society

The instability of silicene on $\operatorname{Ag}(111)$,

A. Acun, B. Poelsema, H.J.W. Zandvliet, R. van Gastel, Appl. Phys. Lett., 103 (26), 263119, 2013

Special note: in the list of most accessed articles of the mentioned journal in 2014

Electron-induced dynamics of heptathioether beta-cyclodextrin molecules,

A. Kumar, R. Heimbuch, K.S. Wimbuch, H. Atesci, A. Acun, D.N. Reinhoudt, A.H. Velders, H.J.W. Zandvliet, Small, 8 (2), 317-322, 2011 


\section{Acknowledgments}

A PhD-trajectory is known for its challenging and stressful characteristics. It is a period of time where hardships are common as many other academicians can probably relate to. While it was not different in my case, my personal life has had tremendous hardships and benevolent moments as well. Looking back at my situation on the $31^{\text {st }}$ of August 2013 (the day before starting my PhDtrajectory) and comparing it to my situation as it is now I get goosebumps due to the intense four years that I experienced. But now, both the PhD-trajectory and personal hardships are over. The feeling of optimism that I have in my grasp now could not have been achieved without a lot of people. By listing everyone's contribution it feels like I am counting my blessings. But at the moment I try to unify all the contributions my mind cannot handle the greatness of the blessing that I have in my life, 'alhamdulillah' (praise be to God).

At first I would like thank my father and my mother for their care. It is the good will in their hearts that kept me optimistic. A special thanks to my sister whom always had a good pair of ears that would listen to me. Without the great characters of these three people I would not even have gone to the university, let alone do a PhD. Thanks, a lot!

During my study there was someone who I always found passionate in his work and inspiring. He is a gentle, supportive and kind person with a great dose of boerenverstand. A great example for the kind of person I would like to become. You made problems look easy, but most importantly, the empathetic power you possess is a rare occurrence nowadays. Thank you for all of your support, inspiration and input, Harold.

A great deal of effort for this thesis and the scientific input has been provided by none other than Arie. I am ambitious to acquire two great features that Arie has, i.e. naastenliefde and a calm, steady and sharp mind. I probably made you angry many times, but you never showed your anger I guess. It was like having a big brother and a supervisor at the same time. Thank you for this privilege.

Bene, there has been something that I have always found fascinating about you 
and it is not only your mindboggling vast knowledge in physics, but also on how you have dealt with hardships and how you were blessed in hard times as you presented in Dresden. The scientific and social ideas that you provided still keep me thinking and hoping for the best. I must admit that I still feel guilty whenever I use je/jij instead of $u$. Thank you for everything.

The gross of the technical expertise I gained on the microscope was inherited through Raoul. The development of the mental and intellectual situation of a PhD-student is typically adapted by observing his first supervisor. I have learned many things from you on how to be in an academic environment from someone that shed guidance and light. Thank you, Raoul.

Besides being supervised, I had the opportunity to be a supervisor myself as well. Floor and Caspar, I have spent almost a year with you. Humor and friendship at their finest are what characterize our bond. You two are very talented and many times I saw you exceeding me. I wish you all the best in your future careers. Annelies and Tom, I am sorry for the annoying theoretical or computational assignments that were really rather challenging, but I saw you both growing enormously while taking on such challenges (which were challenges for me as well). Thank you for the nice time together. From my fellow PhD-colleagues I would like to thank Özlem in particular for being a joyous part of the last phase of my PhD-thesis. Lijie for in the beginning where we had long talks and laughter because of everything in life, basically. And Edwin, a great guy to travel with like back in the days in New Zealand and later in Australia.

Not being able to get off the floor from laughing or just plainly sharing life or scientific stories with Kai, Pantelis, Arzu, Jannis, Rik, Nikolai, Rene and mister Yao were precious moments of time. Thank you all! And last, but not least, the great laughters we shared in our office with Imtiaz, Ali, Jorn and Kevin.

Not only would I like to thank Simone, Hans, Herman, Martin and Stefan for their technical and administrative contributions, but also for who they are and what they mean to me. Seeing them means that you are in safe hands and are guaranteed of support, care and expertise. Thank you for your wonderful contributions.

I spent ten months of my PhD-trajectory in Lausanne, Switzerland. Firstly, I would like to thank my daily supervisor and the very lovely, caring talented Maggie. Though I still feel like I let you down, I want to give you my deepest thanks from within my heart. Without you I would never have been able to find strength in life again.

Then there is a very peculiar friend of mine who arranged the possibility of doing an exchange at EPFL. Someone who I met during my internship carrying companionship not able to be grasped by mind. Your presence and your opinion 
meant and mean so much for me. I find it sad that words written here cannot visualize how good our friendship is.

Fernando, Bart and Daniel were colleagues of mine at EPFL with great dose of humor and talent. Thank you for the wonderful times.

Like stated before this thesis could not have been completed without hope and optimism in my private life. Mehmet, Tolga, Hares and Mirza, thank you for being close friends of mine since our childhood and/or high school period. The (dark) humor, weird talks and all of our experiences accounted for great times.

Next, I would like to thank two brothers of mine, Hasan and Hüseyin. From the beginning of our bachelor studies until the end of the $\mathrm{PhD}$, we have been supportive towards each other and were always good friends. After all this time I am pretty confident that we have developed a lifetime (and beyond) sustainable friendship. Good luck with your defenses soon!

Another study mate with so many similarities and great philosophical and intellectual qualities is Sam. Time never seemed to end when we were hanging out together. Thank you for that!

Then there is a folk that always seems to amaze me with their warmhearted friendships. Somehow they live up to the old Turkish culture as I imagine it to be. My Azerbaijani friends Mahammad, Alekber, Araz, Khayyam, Elmi, Ilqar and Bahruz never failed to breach through my barrier of loneliness and anger. Thank you guys for your warm presence.

Someone who has provided me quite some confidence at times I needed it the most is Mary Tupan Wenno. Thank you for your support and enhancing my identity within the society that we are living in.

Many thanks for bringing a healthy dose of enthusiasm and optimism. And thanks for meneer Wout Smits, a former math teacher of mine from high school. Recently, he passed away, but his persuasion saved me from failing HAVO back in the days.

Also my special thanks to the ICE-PIN football team with whom I had a lot of fun and could detach myself from stress.

And during the hard times in Lausanne, I frequently visited the mosque where I made good warming friends like Fatih, Ismail, Ahmet, Sezai and Sebahattin. Without you my transformation would not have been succeeded.

Last, but not least, I would like to thank my family, my neighbours, my teachers and my partners in crime during conferences (hello, Asif) in general. 